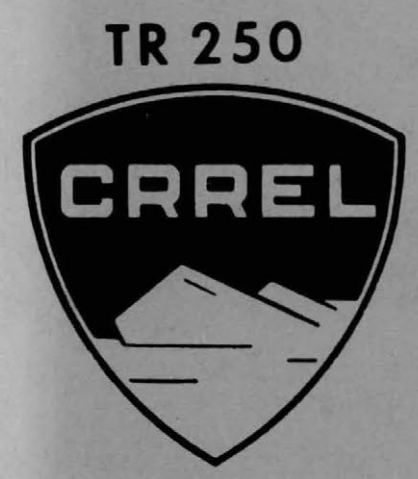

\title{
FREEZING TEST FOR EVALUATING RELATIVE FROST SUSCEPTIBILITY OF VARIOUS SOILS
}

Chester W. Kaplar

\author{
June 1974
}

PREPARED FOR

DIRECTORATE OF MILITARY CONSTRUCTION

OFFICE OF CHIEF OF ENGINEERS

BY

CORPS OF ENGINEERS, U.S. ARMY

COLD REGIONS RESEARCH AND ENGINEERING LABORATORY

HANOVER, NEW HAMPSHIRE 
The findings in this report are not to be construed as an official Department of the Army position unless so designated

by other authorized documents. 


\title{
FREEZING TEST FOR EVALUATING RELATIVE FROST SUSCEPTIBILITY OF VARIOUS SOILS
}

Chester W. Kaplar

\author{
June 1974
}

\author{
PREPARED FOR \\ DIRECTORATE OF MILITARY CONSTRUCTION \\ OFFICE OF CHIEF OF ENGINEERS \\ BY \\ CORPS OF ENGINEERS, U.S. ARMY
}

COLD REGIONS RESEARCH AND ENGINEERING LABORATORY

HANOVER, NEW HAMPSHIRE 
PREFACE

Authority for the investigation reported herein is contained in FY 1964 Instructions and Outline, Military Construction Investigations, Engineering Criteria and Investigations and Studies, Studies of Construction in Areas of Seasonal Frost; Subproject 14, Laboratory Studies of the Effects of Soil Freezing.

The Military Construction Investigations program is conducted for the Directorate of Military Construction, Office of the Chief of Engineers. This investigation was under the technical direction of the Engineering Division of this directorate, Advanced Technology Branch, Civil Engineering Section (Mr. F.B. Hennion, Chief).

Mr. C.W. Kaplar, Research Civil Engineer, Applied Research Branch, conducted the study and prepared this report. The investigation was under the general direction of $\mathrm{Mr}$. K.A. Linell, Chief, Experimental Engineering Division, and the immediate direction of Mr. A.F. Wuori, Chief, Applied Research Branch, U.S. Army Cold Regions Research and Engineering Laboratory (USA CRREL).

This report was technically reviewed by Professor K.B. Woods and Mr. E.F. Lobacz.

The contents of this report are not to be used for advertising, publication, or promotional purposes. Citation of trade names does not constitute an official indorsement or approval of the use of such commercial products. 


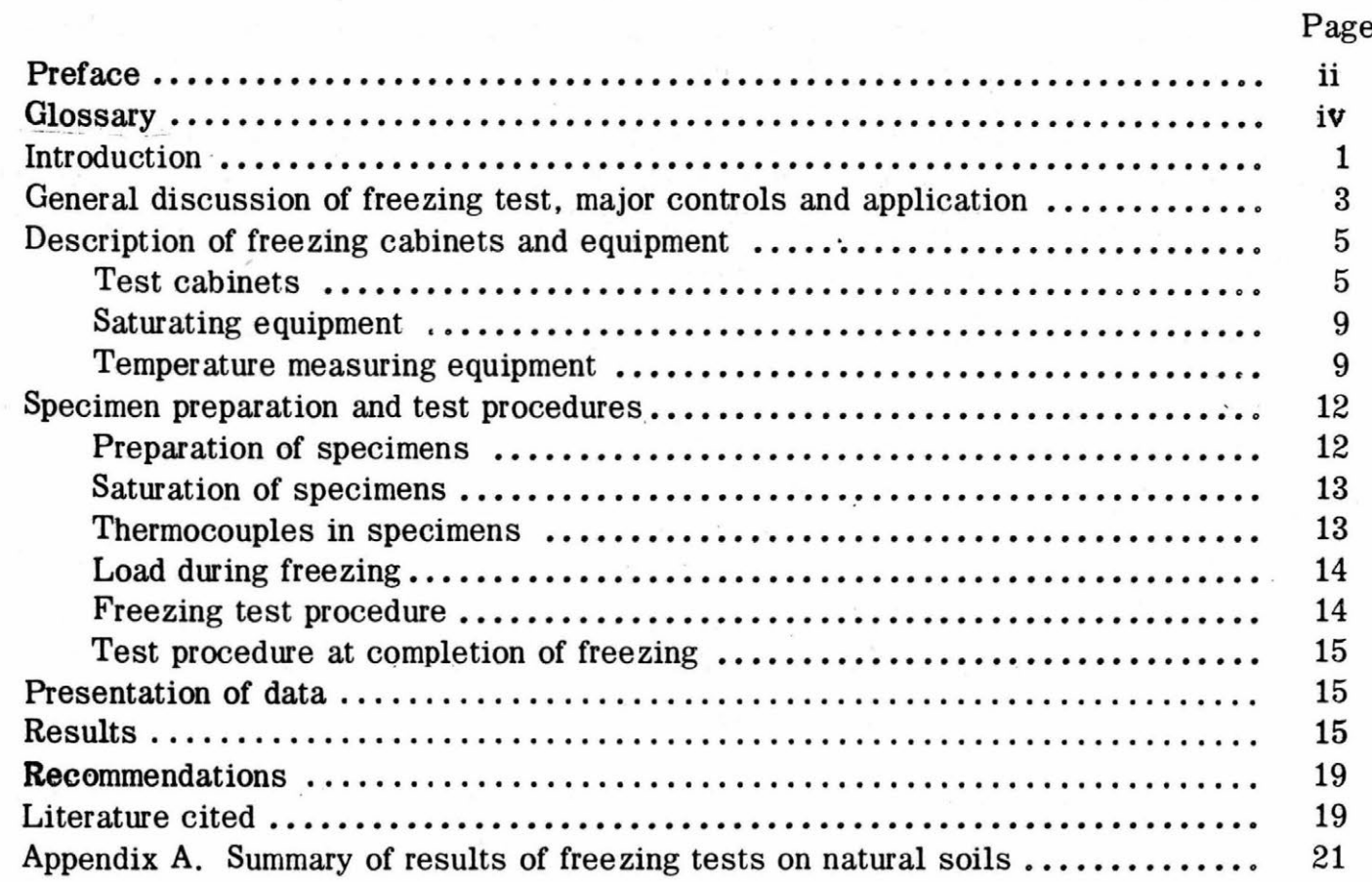

\section{ILLUSTRATIONS}

Figure

1. Inside-tapered freezing cell $\ldots \ldots \ldots \ldots \ldots \ldots \ldots \ldots \ldots \ldots \ldots \ldots \ldots \ldots, 3$

2. Freezing cell disassembled ................................ 4

3. Freezing cabinets in coldroom ........................... 6

4. Details of soil freezing cabinet $\ldots \ldots \ldots \ldots \ldots \ldots \ldots \ldots \ldots \ldots \ldots \ldots \ldots, 7$

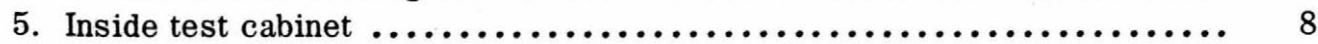

6. Soil specimen undergoing saturation in freezing cell $\ldots \ldots \ldots \ldots \ldots \ldots \ldots .10$

7. Temperature-measuring equipment for use with thermocouples $\ldots \ldots \ldots \ldots \ldots 10$

8. Typical record of coldroom temperature control ................... 11

9. Typical record of freezing cabinet temperature control $\ldots . . . \ldots \ldots \ldots \ldots \ldots 11$

10. Tapered steel molding cylinder $\ldots \ldots \ldots \ldots \ldots \ldots \ldots \ldots \ldots \ldots \ldots \ldots \ldots \ldots$

11. Soil specimen in freezing cell with 0.5 -psi surcharge load ............ 14

12. Frozen soil specimen before and after freezing $\ldots \ldots \ldots \ldots \ldots \ldots \ldots \ldots . \ldots$

13. Typical soil gradation curves ............................ 17

14. Typical temperature and heave data for specimen K-1 - Open-system test .. 18

15. Typical water content vs specimen height..................... 19

Table

\section{TABLES}

I. Typical physical properties of the samples received $\ldots \ldots \ldots \ldots \ldots \ldots \ldots, 17$

II. Typical summary of specimen freezing test data $\ldots \ldots \ldots \ldots \ldots \ldots \ldots \ldots .17$ 


\section{GLOSSARY}

Definitions of the following terms used in this report were taken principally from a list prepared and approved by the Highway Research Board (HRB) Committee on Frost Heave and Frost Action in Soils. ${ }^{5}$

Frost action. A general term for the freezing and thawing of moisture in materials and the effects on these materials and on structures of which they are part or with which they are in contact.

Frost heave. The raising of a surface due to the formation of ice in the underlying soil.

Percentage of heave. The ratio, expressed as a percentage, of the amount of heave to the depth of frozen soil before freezing.

Frost-susceptible soil. Soil in which significant (detrimental) ice segregation occurs when the requisite moisture and freezing conditions are present.

Ice segregation. The growth of ice as distinct lenses, layers, veins and masses in soils, commonly oriented normal to the direction of heat loss.

Ice lenses. Ice formations in soil occurring essentially parallel to each other, generally normal to direction of heat loss, and commonly in repeated layers.

Open system. A condition in which free water in excess of that contained originally in the voids of the soil is available to be moved to the surface of freezing, to form segregated ice in frost-susceptible soil.

Closed system. A condition in which no source of free water is available during the freezing process beyond that contained originally in the voids of soil.

Rate of heave.* The average rate of heave, in millimeters per day, determined from a representative portion of a plot of heave versus time, in which the slope is relatively constant, and during which the penetration of the $32^{\circ} \mathrm{F}$ isotherm is at the relatively uniform rate of between $1 / 4$-in. and $1 / 2-$ in./day. Rate of heave is averaged over as much of the heave-versus-time plot as practicable, but the minimum number of days used for a determination is five. This measure of frost susceptibility is used in open system tests only and pertains to data presented and discussed in this paper.

Degree-hours. The degree-hours for any one hour equals the difference between the average hourly air temperature and $32^{\circ} \mathrm{F}$. The degree-hours are minus when the average hourly temperature is below $32^{\circ} \mathrm{F}$ (freezing degree-hours) and plus when above (thawing degree-hours).

*Not on list of definitions prepared by HRB Committee on Frost Heave and Frost Action in Soil. 


\title{
FREEZING TEST FOR EVALUATING RELATIVE FROST SUSCEPTIBILITY OF VARIOUS SOILS
}

\author{
by \\ Chester W. Kaplar

\section{INTRODUCTION}

The frost susceptibility of soils has been of great concern to civil engineers and most road and highway designers for many years. In spite of advances that have been made in the knowledge of frost action, there is still much evidence of annual frost damage and deformation of road surfaces in many northern areas. Other manifestations of frost damage are the tilting of culvert walls, shallow bridge piers, and abutments; and the jacking out of guard posts and utility poles. Much of this damage is found on the older roads built before World War II; however, some of the newer roads of the interstate highway system are still plagued with the perennial frost-heaving problem and its consequences. One reason for this may be that the people concerned with building highways do not fully understand or are unable to recognize the soil types that are potentially frost susceptible.

Other than the Casagrande rule-of-thumb criteria, ${ }^{4}$ which were not meant to be absolute indicators, but engineering guidelines, no simple, proven laboratory or visual method has been developed for recognizing or evaluating the frost potential of a given soil. The Casagrande criteria, which are based on the particle size distribution characteristics of a soil, have proved very useful in identifying correctly about $85 \%$ of the frost-susceptible soils encountered. ${ }^{10}$ Some soils may show undesirable frost-heaving characteristics, with less than $3 \%$ of their particles by weight smaller than $0.02 \mathrm{~mm}$ in size. Perhaps the greatest objection to the Casagrande rule-of-thumb criteria is that some nonfrost-susceptible soils containing more than $3 \%$ of particles finer by weight than $0.02 \mathrm{~mm}$ might be rejected as undesirable. This is an important point since known sources of good-quality non-frostsusceptible base course materials are fast becoming depleted or are already very scarce-in many localities; it is necessary that the best possible use be made of all available natural material. Therefore, a freezing test procedure is required that will enable the relative frost susceptibility of various natural soils on many major construction jobs to be evaluated. The freezing test procedure described herein, although time-consuming, has been successfully used for this purpose.

During World War II the need for stable and exceptionally smooth runways for high-speed aircraft and the rapidly increasing airplane wheel loads of unprecedented proportions made it imperative that runways be designed to accommodate these loads under all conditions of soil type and in all seasons of the year. This necessitated a better knowledge of soil types and conditions conducive to frost action. Numerous field investigations were conducted at various northern locations throughout the country and a program of laboratory studies was subsequently started by the Corps of Engineers to obtain a better understanding of the variables involved in frost action phenomena and to better identify and classify frost-susceptible materials. ${ }^{11}$ The author participated in the field work 
conducted in northern New England. To conduct the laboratory studies, the coldroom and freezing cabinets available in Pierce Hall at the then Harvard Graduate School of Engineering (presently the Division of Engineering and Applied Physics) were leased through arrangements with Dr. A. Casagrande, who was retained as a consultant on the studies.

In 1950 a new coldroom and nine special freezing cabinets were installed at the Frost Effects Laboratory,* New England Division, Corps of Engineers. ${ }^{12}$ The author was assigned the major responsibility of designing the freezing cabinets [which were modeled somewhat after the Harvard (Casagrande) equipment] and of formulating a technique and procedures for conducting frostsusceptibility tests to achieve the purpose of these studies. These cabinets were designed to freeze specimens up to 6 in. high. Later, a tenth cabinet was added to accommodate specimens up to 42 in. high.

It was desired to set up a test procedure to evaluate the full potential of frost activity that a soil was capable of exhibiting under conditions simulating those of nature. Studies of numerous field data ${ }^{11}$ from various localities throughout the country, including observations of frost penetration under runways, heave measurements, and visual observations of ice lens formation and distribution in test pits, showed that frost penetration was fairly continual during the winter and that ice lenses became more numerous and thicker when the frost penetration rate was relatively slow, i.e., about $1 / 4 \mathrm{in} . /$ day. This rate of frost penetration was adopted for use in the laboratory studies with the approval of the special Board of Consultants for Frost Studies, Office, Chief of Engineers, Department of the Army: Professors A. Casagrande, P.D. Rutledge, and K.B. Woods. Other test conditions agreed upon were specimen height of $6 \mathrm{in}$., full initial saturation and maintenance of water supply at the base of specimens during open-system test, 0.5 psi surcharge pressure, and unidirectional freezing of specimens from the top down with the specimens insulated on the sides.

Initially during these studies test specimens were compared on the basis of percentage of heave at the end of freezing. It was frequently noted that large differences in percentages of heave were recorded for the same soils even though the average heave rates appeared to be reasonably similar. For example, it was observed that the percentage of heave for a 6 -in.-high specimen frozen in 8 days at the rate of $3 / 4$ in./day when control of the rate of freezing was poor was considerably less than that for a 6-in.-high specimen frozen in 24 days at the slow rate of approximately $1 / 4$ in./ day. Because of Beskow's ${ }^{3}$ observation that heave rate was relatively independent of frost penetration, $\uparrow$ the rate of heave was adopted as a measure of frost potential, and the following relative frost susceptibility classification, based on average heave rate for the test conditions specified and for rates of freezing between $1 / 4$ and $1 / 2$ in./day, was proposed by Dr. Casagrande:

\begin{tabular}{|c|c|}
\hline $\begin{array}{c}\text { Average rate } \\
\text { of heave } \\
(\mathrm{mm} / \mathrm{day}) \\
\end{array}$ & $\begin{array}{l}\text { Relative frost } \\
\text { susceptibility } \\
\text { classification }\end{array}$ \\
\hline $0-0.5$ & Negligible \\
\hline $0.5-1.0$ & Very low \\
\hline $1.0-2.0$ & Low \\
\hline $2.0-4.0$ & Medium \\
\hline $4.0-8.0$ & High \\
\hline er than 8.0 & Very high \\
\hline
\end{tabular}

\footnotetext{
*Redesignated the Arctic Construction and Frost Effects Laboratory in 1952 and merged wi th the Snow, Ice and Permafrost Research Establishment, U.S. Army Corps of Engineers, in 1961 to form the U.S. Army Cold Regions Research and Engineering Laboratory.

†Subsequent studies at USA ACFEL and USA CRREL ${ }^{7}$ showed that the rate of freezing of specimens having some boundary conditions, as indicated in this report, did affect the rate of heaving; i.e. heaving rate increased with increased freezing rate.
} 
This report describes the standard freezing test designed for the Corps of Engineers by the author, with emphasis on the procedures and equipment, and contains a summary of the results of frost-susceptibility tests conducted on natural soils up to 1970.

\section{GENERAL DISCUSSION OF FREEZING TEST, MAJOR CONTROLS AND APPLICATION}

The laboratory technique which has been used by the U.S. Army Corps of Engineers since 1950 to evaluate the relative frost susceptibility of various soils is similar to techniques used earlier by Taber, ${ }^{9}$ Beskow, ${ }^{3}$ Casagrande, ${ }^{4}$ Winn and Rutledge, ${ }^{14}$ and others, except that it has been used on much larger specimens to accommodate gravelly soils. The soil containers initially used were waxed carboard cylinders and standard acrylic tubes, approximately 6 in. in diameter and 6 in. in height. Subsequently a Lucite soil cell (Fig. 1) with a number of improvements was designed. This new cell contained a slightly tapered, vertical inner wall (1/4-in. greater diameter at top), which significantly reduced frictional wall resistance to heaving, a condition which it is believed may have seriously affected some previous test results to an unknown and variable degree. A disassembled cell is shown in Figure 2.

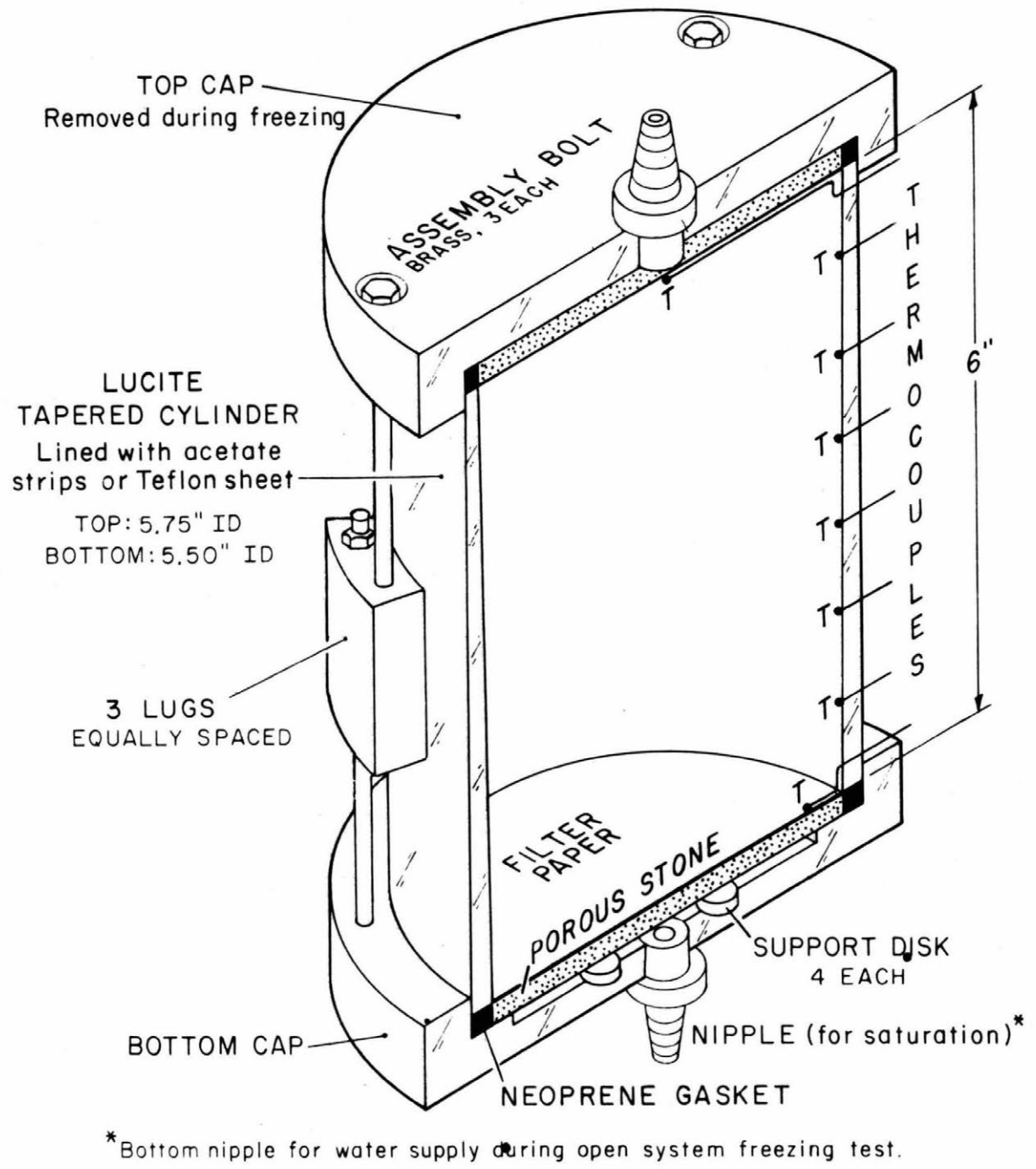

Figure 1. Inside-tapered freezing cell. 


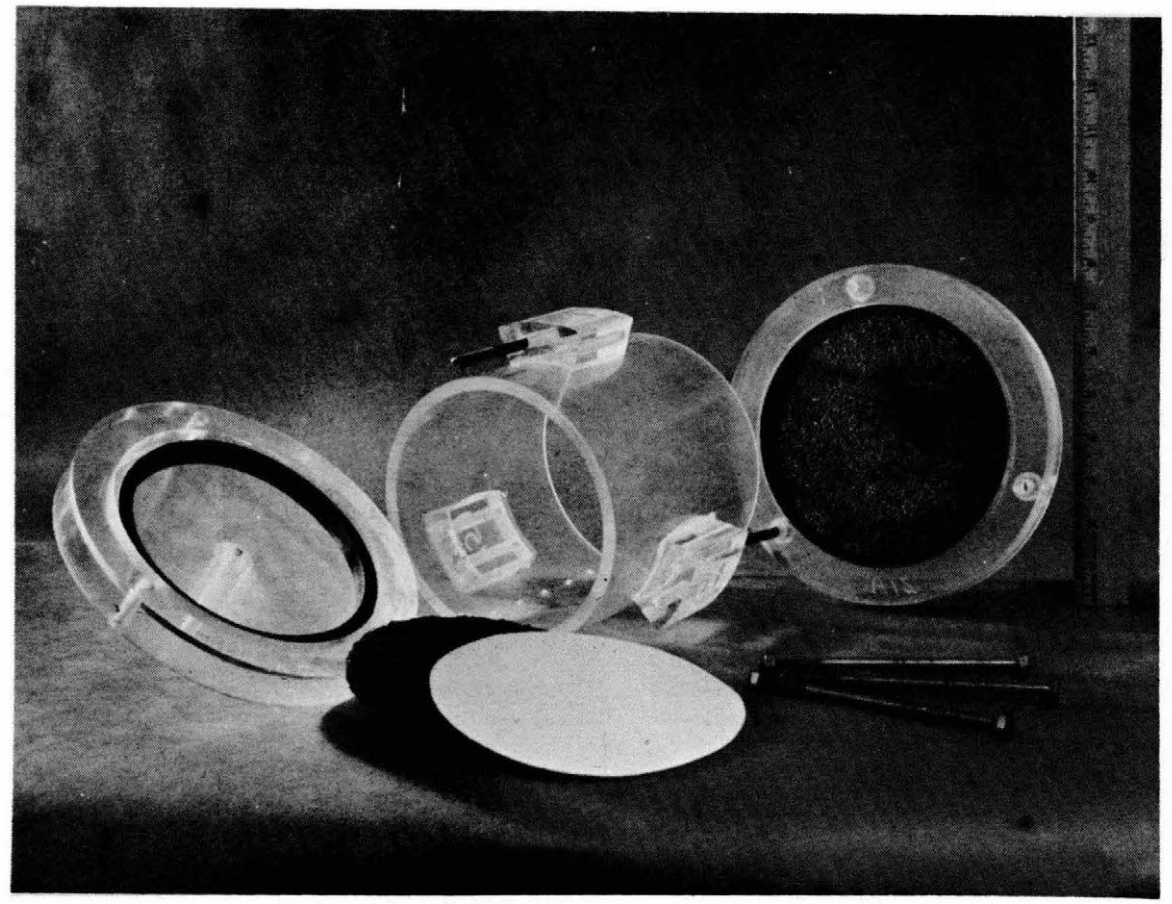

Figure 2. Freezing cell disassembled.

The soils are generally compacted to densities normally encountered in pavement construction, and the rate of penetration of freezing temperature into the specimens of about $1 / 4$ to $1 / 2$-in./day is considered to be representative of field conditions during a large part of the winter. Except for special closed-system tests (no external water supplied), an unlimited supply of water is provided at the base of the specimens during freezing. In the field this corresponds to an extremely pervious acquifer only a short distance below the plane of freezing. This is a severe condition, and it results in virtually the maximum amount of ice segregation and heave that the soil can exhibit in a pavement system.

The freezing test briefly described above has been designed to subject the soil to a very severe combination of conditions conducive to frost action. It is the only laboratory test established to date that has been effectively used to evaluate the relative frost susceptibility of soils and that has been used in support of major engineering design and construction programs by some states, the Corps of Engineers, and other Federal departments and agencies. The main control used in the tests is the rate of frost penetration, as stated previously. The observed effect on the specimens, the measure of which is used as a criterion for comparing the relative degree of frost susceptibility of different soils, is the rate of heaving or ice lens growth. The use of a positive freeze penetration into a soil specimen containing fine-grained particles and moisture activates thermodynamic energy such that heaving takes place and soil moisture is caused to migrate to the freezing plane. This rate of moisture movement (and ice lens growth) is governed by the unique characteristics of the soil fabric and by other fixed conditions of the test, such as dry unit weight, overburden load, position of free water surface, and freezing rate; it is thus considered an effective indicator of the relative frost-heaving potential of the soil.

The heave rate results obtained in the laboratory tests should not be considered as quantitatively representative of the actual heave rates to be expected in the field during a winter. The results, however, are considered to give a satisfactory measure of the relative frost susceptibility 
potential of soils under severe but feasible field conditions with the possible exception of unweathered clays, which may show unduly low heave for at least the first cycle of freezing.

The evaluation given by the freezing test described is empirical in nature. The average rate of heave measured in the tests does not represent a simple and fundamental physical value, since such factors as overburden pressure and moisture availability at the plane of freezing vary continually during the test. Nevertheless, the test and the results obtained have been useful in many ways, for example:

1) in selecting the least frost-susceptible material for construction use when several choices are available (natural gradations containing 2-in.-diam maximum size stones can be tested)

2) in evaluating the frost potential of in-situ soil or soil previously used for construction

3) in evaluating the effectiveness of soil additives, such as coarse inclusions, metallic and fibrous reinf orcement, cements, void pluggers, salts and chemicals, in studies of frost inhibitors and modifiers

4) in conducting laboratory studies on soils to determine the effect of various soil parameters, such as load pressure, dry unit weight, and initial degree of saturation, on the frost behavior of the soils.

Thus, this freezing test is undoubtedly only an initial step toward an ultimate rational test procedure that will evolve from the research now in progress at USA CRREL and other laboratories because the basic controlling factors responsible for ice segregation have not been incontrovertibly established. However, the present test has been used successfully by the Corps of Engineers for a considerable number of years. The problem of controls has challenged some of the ablest soil physicists and physical chemists. A number of theories have been advanced in recent years but none has received wide acceptance or experimental confirmation. Also, a mathematical formulation for heave prediction for given soil and field conditions has not yet been developed. In the interval, engineering needs must be met with the most versatile and adaptable empirical procedures.

Recently a test that measures the heaving pressure that develops at a freezing plane under static equilibrium temperature conditions ${ }^{6}$ has been proposed by some investigators as a measure of frost susceptibility potential. This test, though shorter in duration than the test described in this report,* cannot be used to evaluate realistically 1) the frost-heave potential of silty sands and gravelly soils, and fine-grained soils containing coarse inclusions; and 2) the effects of a) chemicals and additives (such as cements and binders, and waterproofers), b) load pressures in reducing heaving to acceptable levels, and c) moisture content, permeability, layered structure, etc., on the frost susceptibility of soils.

The test described herein was designed to evaluate frost susceptibility behavior under all the above-mentioned conditions.

\section{DESCRIPTION OF FREEZING CABINETS AND EQUIPMENT}

\section{Test Cabinets}

This test procedure requires that test specimens be initially cooled to a temperature of $38-40^{\circ} \mathrm{F}$ and that this temperature be maintained at the base of the specimen while a variable freezing temperature is applied at the top to produce the desired rate of freeze $\left(32^{\circ} \mathrm{F}\right)$ penetration.

* Preliminary experiments have been performed at USA CRREL ${ }^{7}$ to speed up the frost susceptibility testing while retaining the criterion of heave rate as a measure of soil evaluation. 


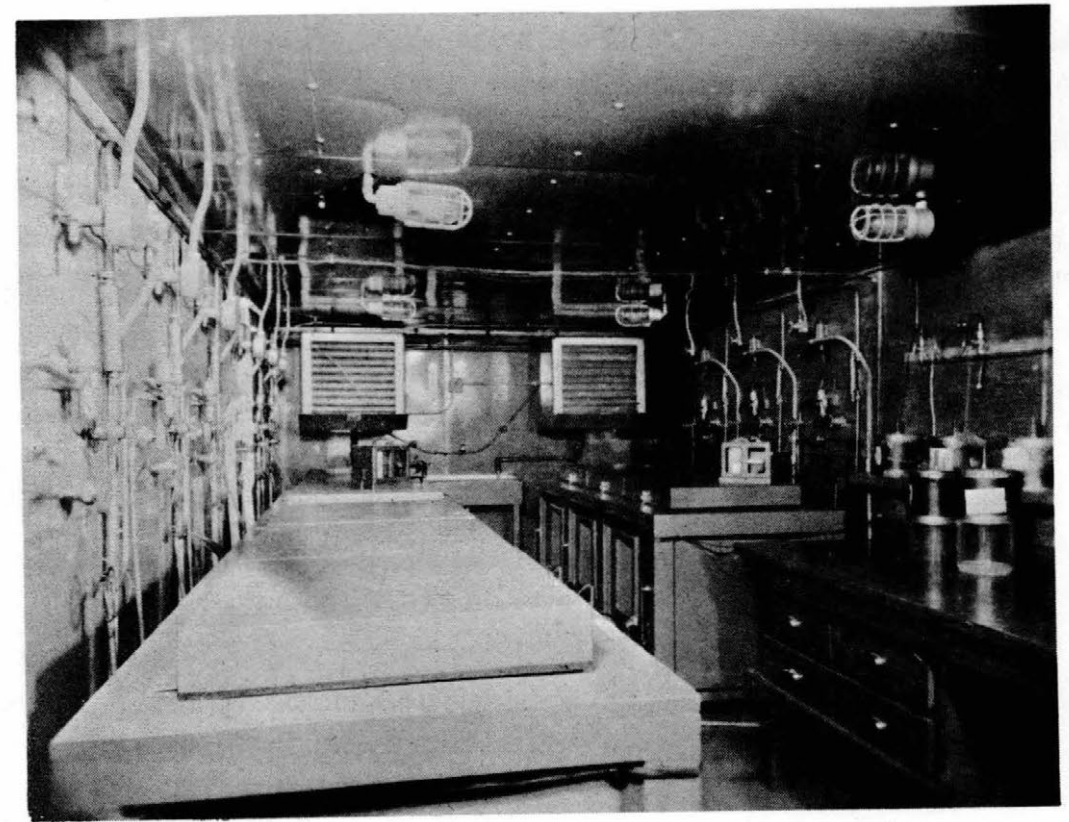

a. Freezing cabinets in $38^{\circ} \mathrm{F}$ coldroom.

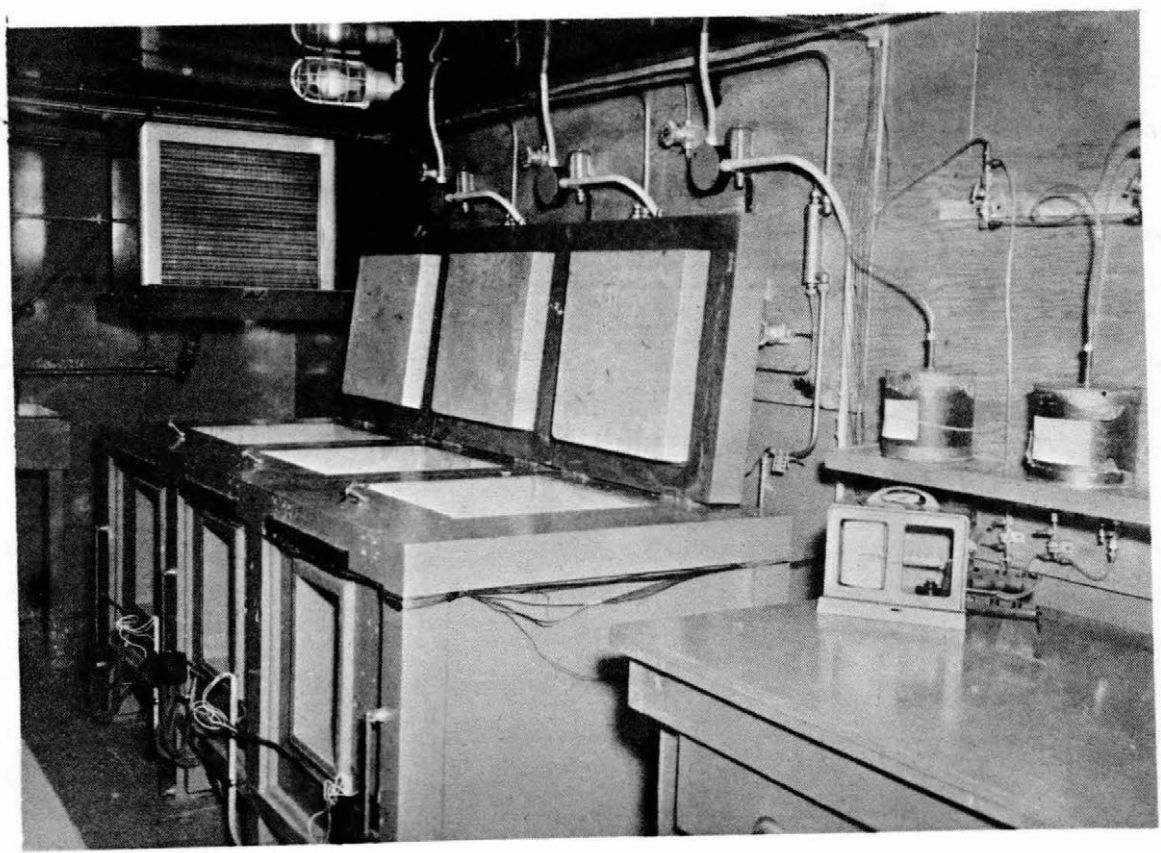

b. Closeup of a group of freezing cabinets. Two specimens undergoing saturation on right, prior to freezing.

Figure 3. Freezing cabinets in coldroom. 


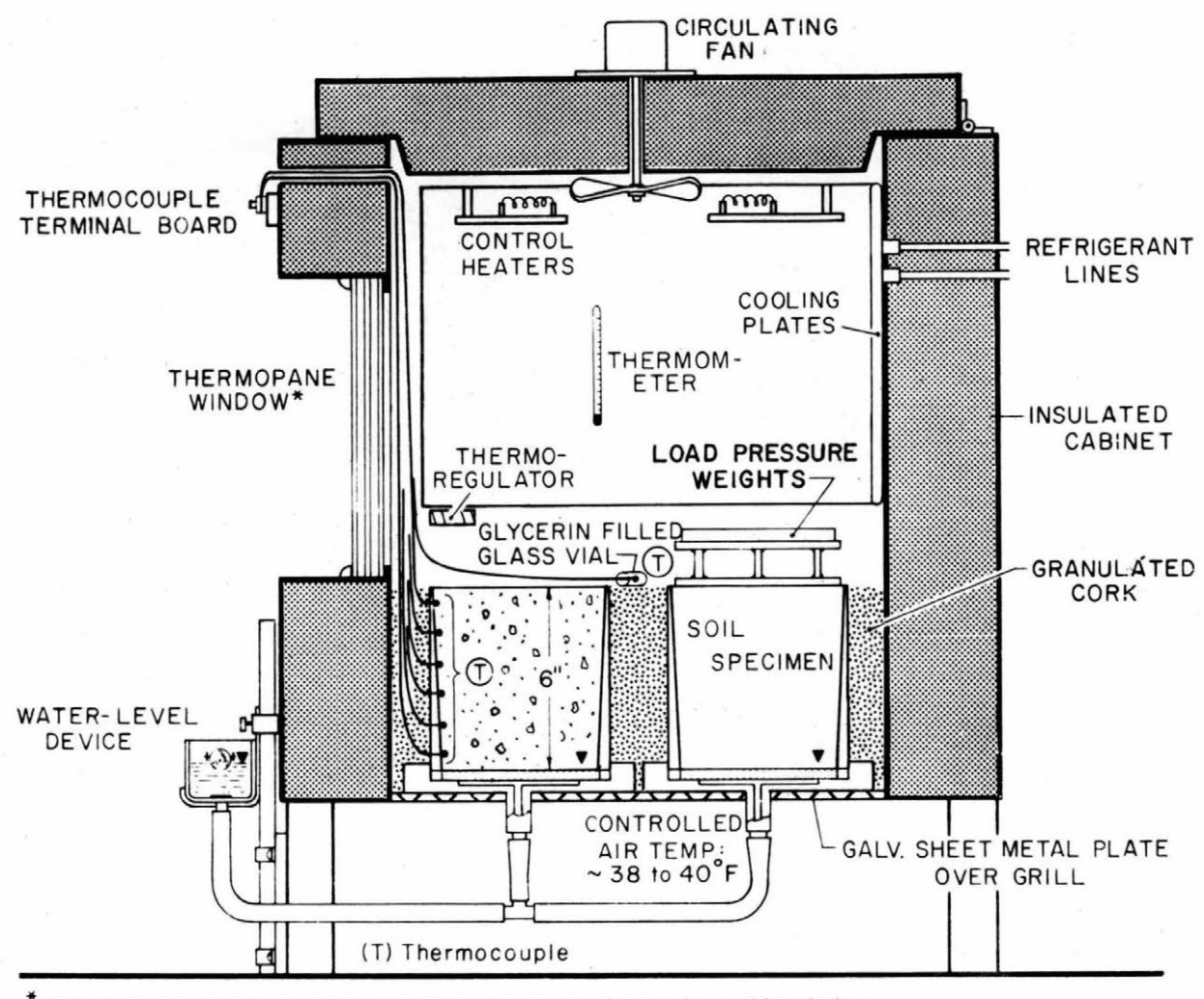

${ }^{*}$ Glass thermometer located in test cabinet may be viewed from this window.

Figure 4. Details of soil freezing cabinet.

Because of the number of cabinets (10) required to conduct the Corps of Engineers' frost investigations program, economy and efficiency considerations dictated the necessity of a walk-in coldroom maintained at $38-40^{\circ} \mathrm{F}$ to house the test cabinets and provide the temperature needed at the base of the specimens in the cabinets. (For individual cabinets a walk-in coldroom would be impractical and a separate refrigeration unit could be used to cool the space beneath the cabinet. ${ }^{7}$ Free-standing cabinets outside a coldroom would also require better thermally insulated walls to reduce cooling load in the cabinets.)

The cabinets used in the Corps of Engineers' investigations are constructed in groups of three with common partitions between the individual units (Fig. 3). Refrigerant is provided separately to each cabinet by 1/4-hp air-cooled units placed outside of the coldroom. The cabinets are designed to operate at temperatures ranging from ambient coldroom temperature down to $\approx-30^{\circ} \mathrm{C}\left(-20^{\circ} \mathrm{F}\right)$. The temperature in each cabinet is controlled by an appropriate thermoregulator that is placed at approximately specimen level and has an accuracy of $\pm \approx^{1 / 4^{\circ}} \mathrm{C}\left(1 / 2^{\circ} \mathrm{F}\right)$. A fan is used to obtain temperature uniformity in the cabinet.

The cabinets have inside dimensions of 19 in. by $19 \mathrm{in.}$ and can accommodate soil specimens up to 12 in. high. A schematic of a cabinet is given in Figure 4. A typical prepared specimen, 6 in. high, is also shown in Figure 4. Each cabinet is equipped with a hinged cover on top, facilitating access to the cabinet for observations and necessary measurements with minimum disturbance to the cabinet temperature. As many as 36 samples, 11/4 in. in diameter, may be tested in each cabinet at one time. A more recent improvement has been the installation of thermostatically controlled electric heating coils on the inside of covers (Fig. 4) to improve the overall temperature control. 


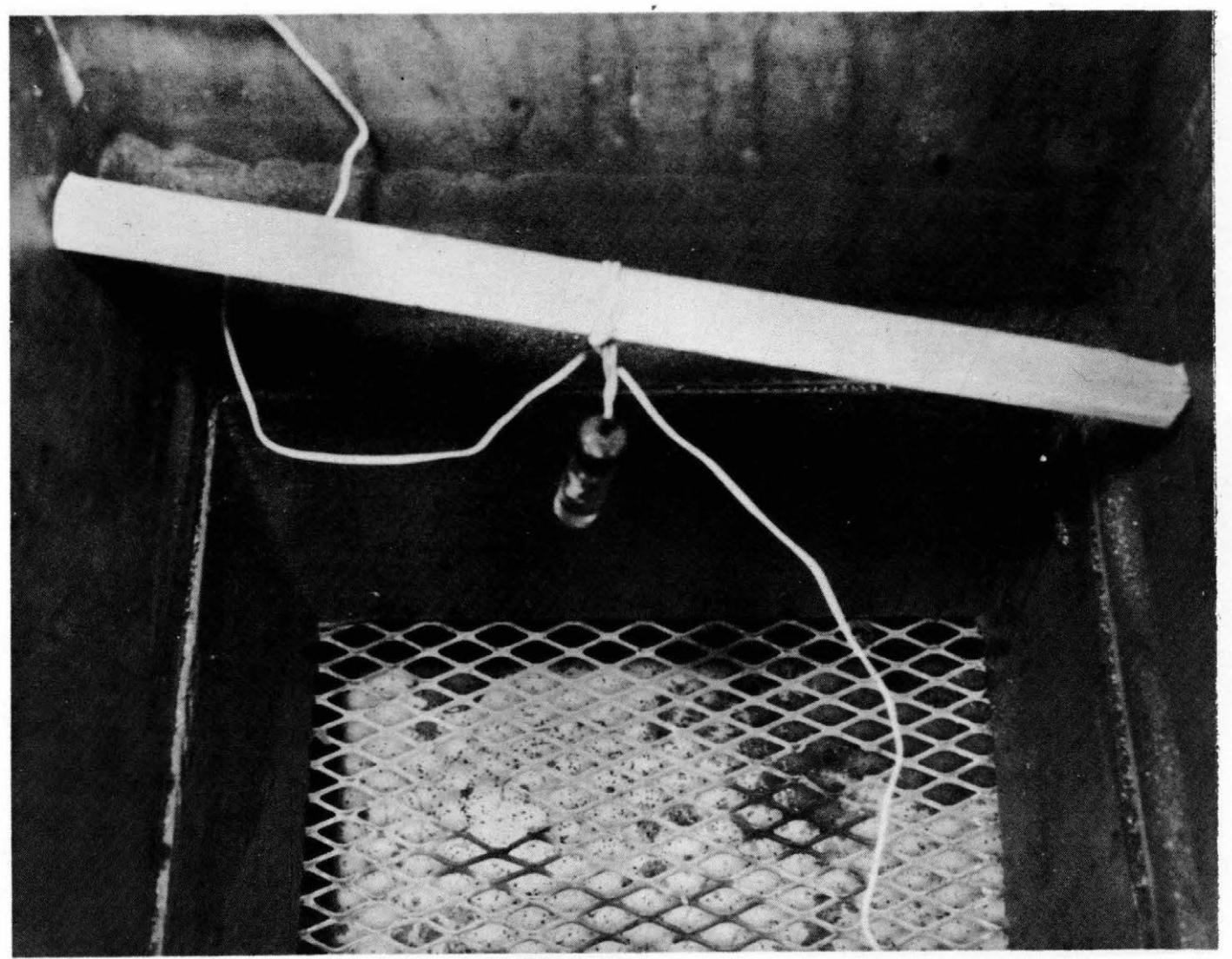

a. Open grillwork at bottom of test cabinet for supporting samples.

Figure 5. Inside test cabinet.

The bottoms of the test cabinets consist of open grillwork (Fig. 5a) to allow the coldroom temperature to be applied to the bottoms of the soil specimens being tested while the tops of the specimens are being subjected to the cabinet temperatures. During freezing tests cabinet temperatures are gradually adjusted in small daily decrements to produce a rate of $32^{\circ} \mathrm{F}$ frost penetration (at the rate of approximately $1 / 4 \mathrm{in./day)}$ into the samples, simulating natural field conditions. Specimens are placed on filter paper over saturated porous disks in individual freezing cells to which de-aired water is continually supplied during the test unless test conditions call for a closed system. These cells rest on grillwork (Fig. 5b). The space between the samples is insulated with granulated cork (Fig. 4). The inside of the test cabinet is cooled by passing the refrigerant through single embossed plates inside a 14-in.-wide zinc-coated copper cooling plate fitted to three sides of the cabinet, beginning 13 in. from the bottom and continuing to the top. A thermopane window occupies the fourth (front) side. Facilities for furnishing de-aired water to any specimen and for maintaining a definite water level within the specimens in a test cabinet are provided by a constant water level device which is adjustable over the height of the sample (Fig. 4). 


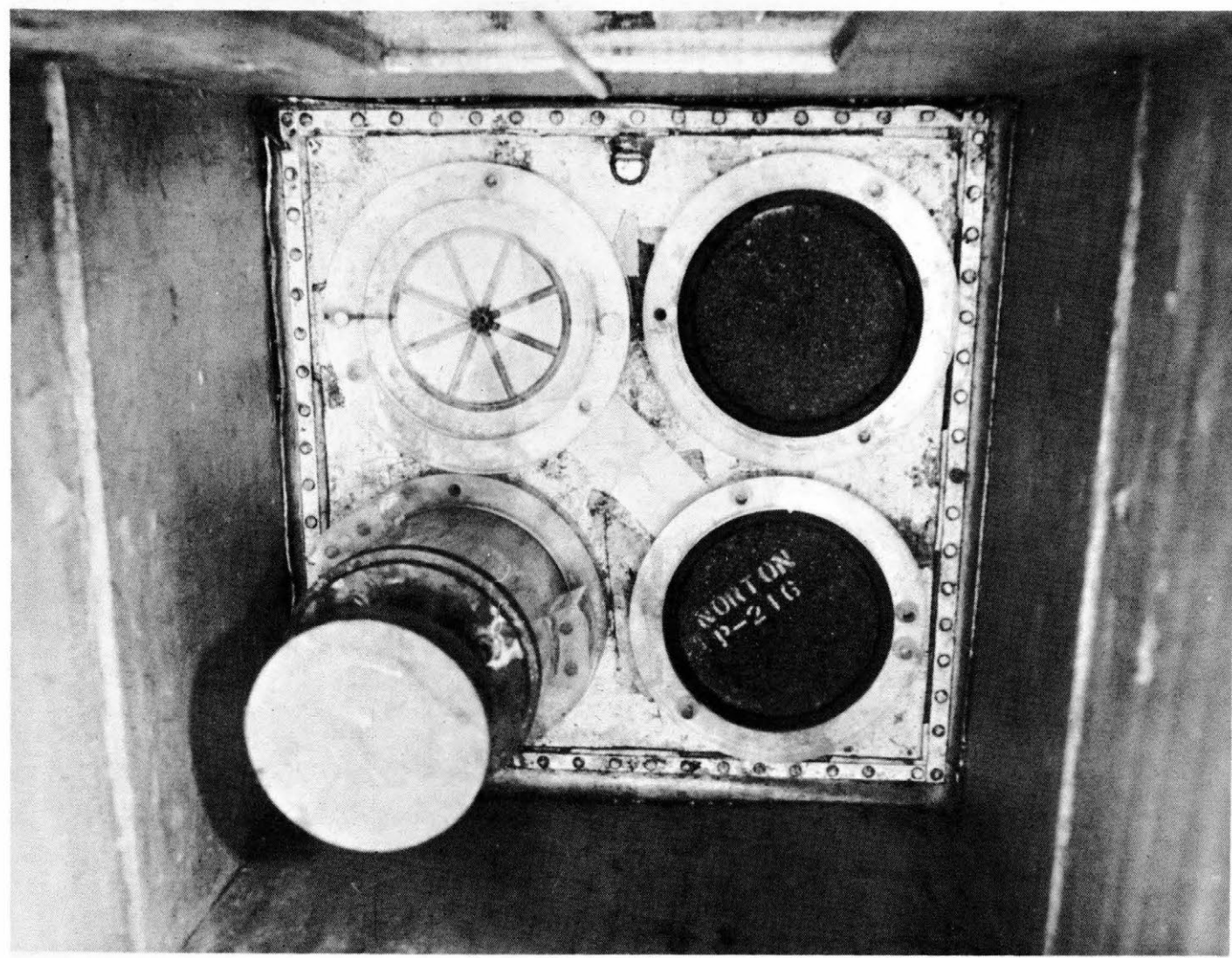

b. Bottom of test cabinet, showing grillwork, with four sample receptacles. One is empty, two have porous stone inserted, and a fourth is complete with soil specimen and surcharge weights.

Figure 5 (Cont'd).

\section{Saturating Equipment}

The coldroom contains separate facilities for saturating soil specimens to be tested. Several specimens in freezing cells may be saturated at one time. Specimens are first connected to a vacuum manifold which de-airs the tops and bottoms of the specimens. De-aired water is then supplied from a second manifold which is connected to the bottoms of the specimens. The de-aired water is controlled by means of suitable valves. A specimen undergoing saturation is shown in Figure 6 .

\section{Temperature Measuring Equipment}

The temperatures in soil specimens are measured by means of copper-constantan thermocouples that can be placed at any desired point in the specimens. The thermal electromotive force produced by the thermocouples is measured by electrical instruments consisting of a standard cell, a sensitive galvanometer, and a Leeds and Northrup type $\mathrm{K}-2$ potentiometer, located immediately outside the coldroom. Temperatures can be read accurately to $0.05^{\circ} \mathrm{F}$. A toggle switchbox enables any one of 100 thermocouples to be placed rapidly in the measuring circuit. This equipment (Fig. 7 ) is 


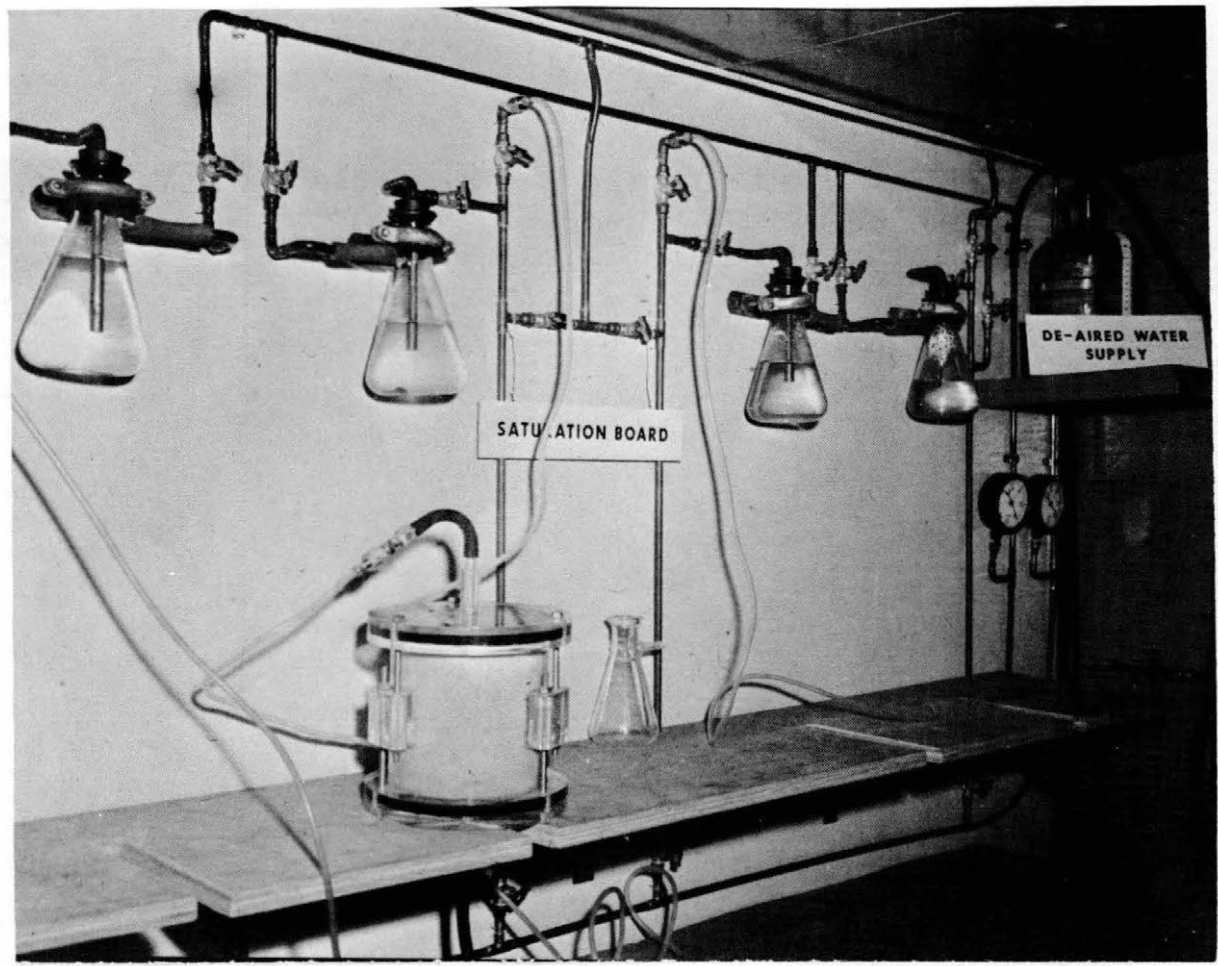

Figure 6. Soil specimen undergoing saturation in freezing cell.

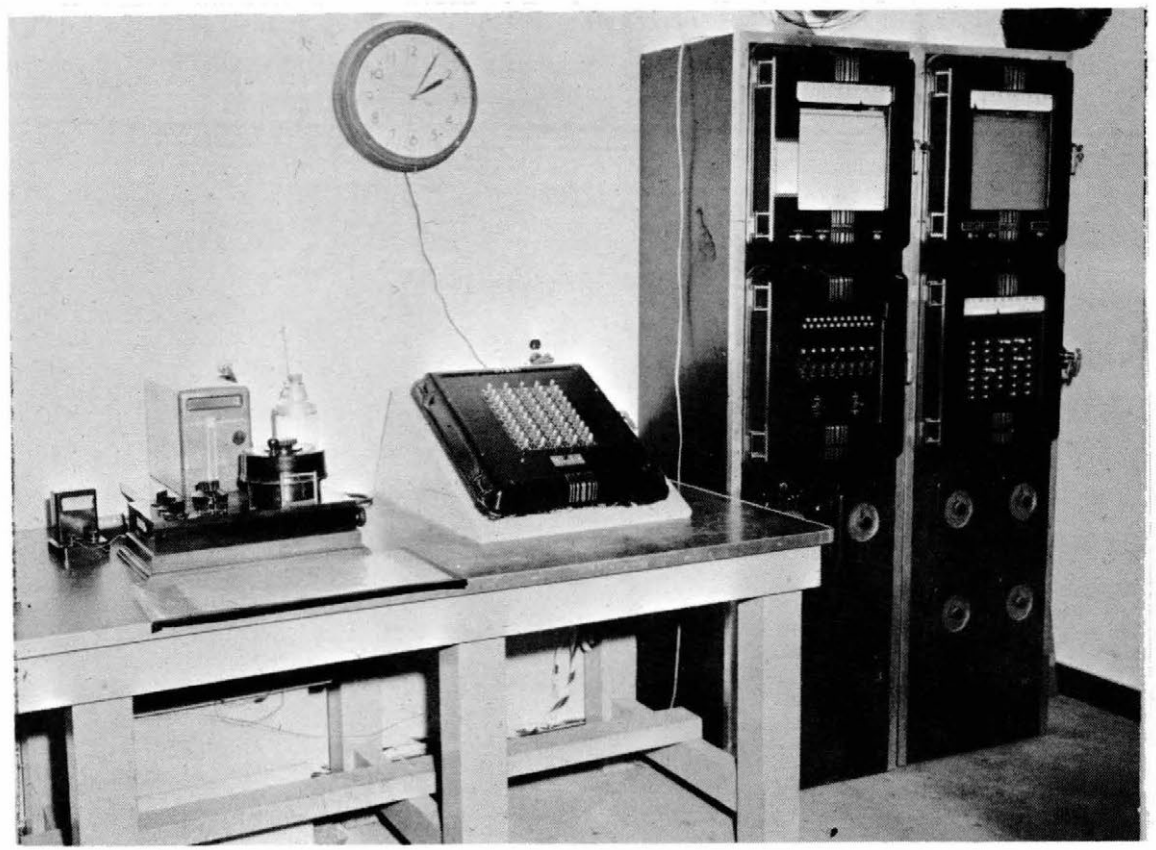

Figure 7. Temperature-measuring equipment for use with thermocouples. 


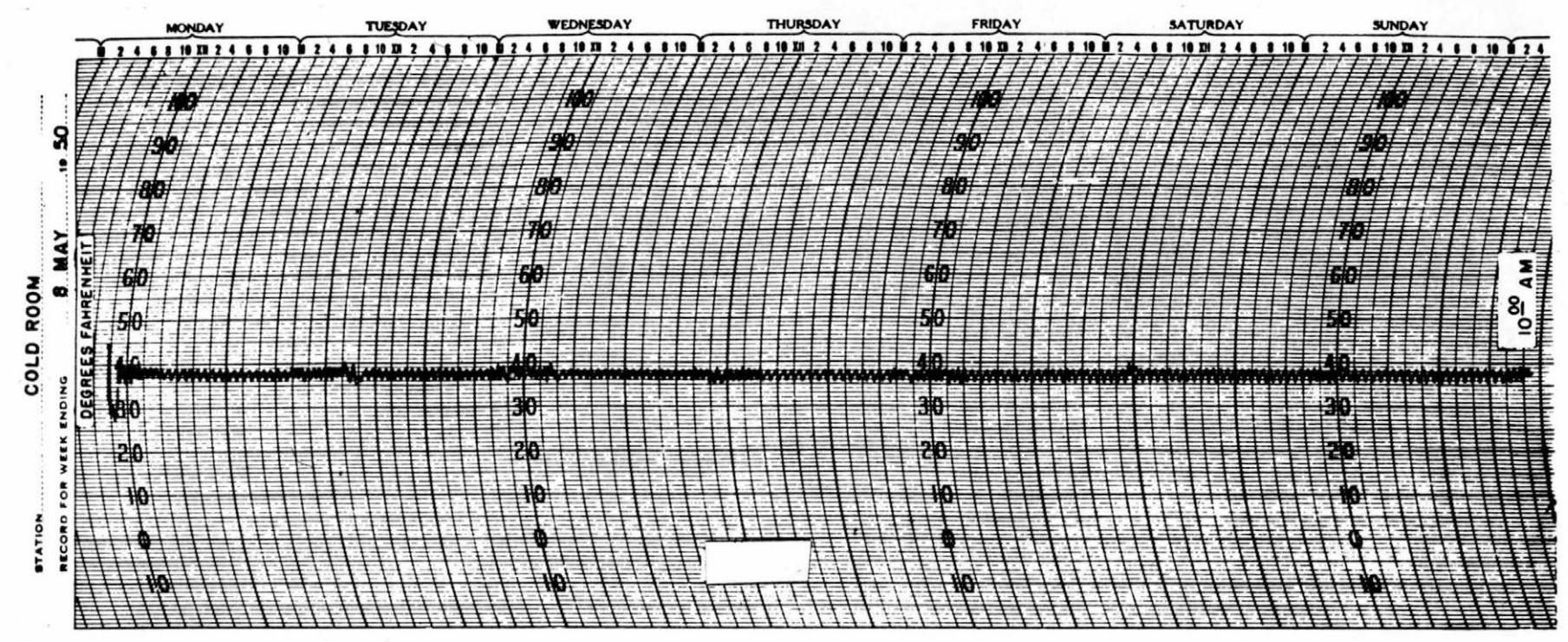

Figure 8. Typical record of coldroom temperature control.

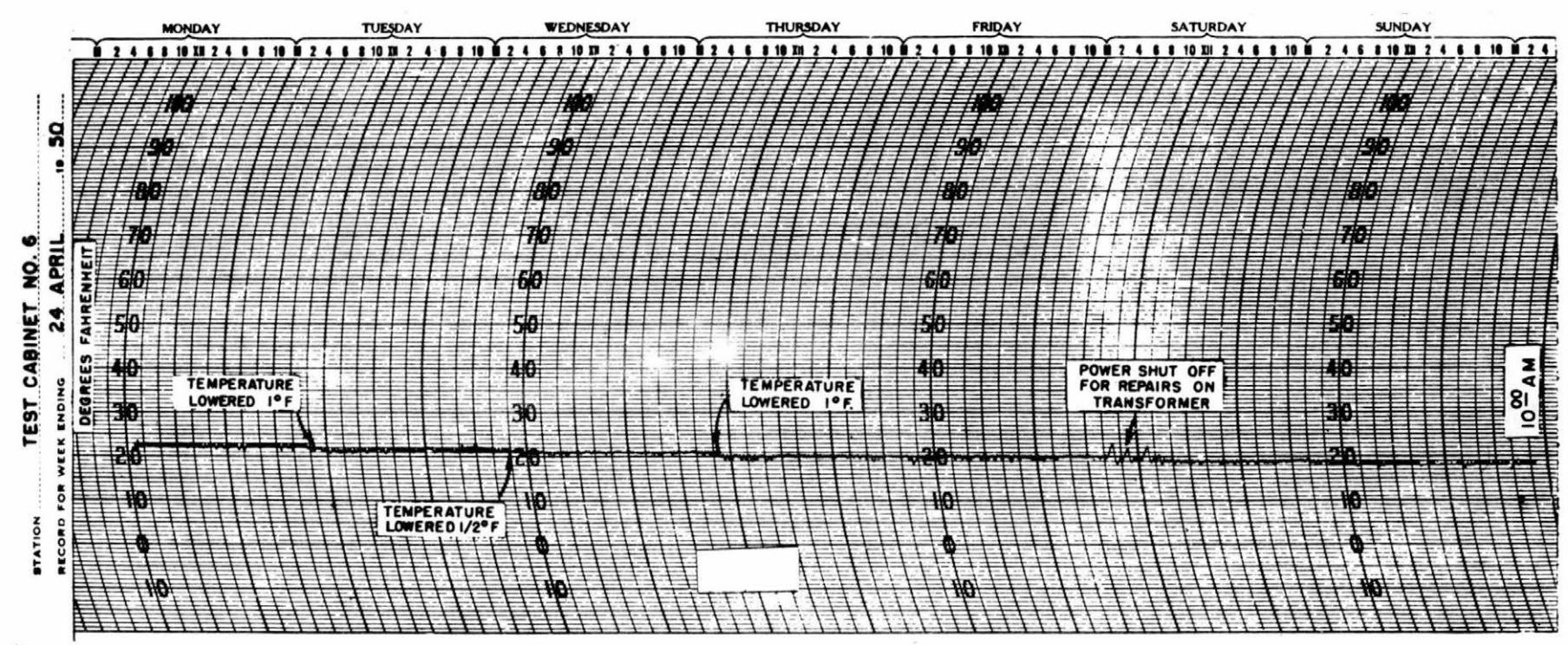

Figure 9. Typical record of freezing cabinet temperature control. 
conveniently located in an instrument room outside the coldroom wall. Each test cabinet is equipped with a glass thermometer that can be read through the thermopane window. However, a close check of each cabinet temperature is maintained by means of a thermocouple inserted into a glycerinfilled, rubber-stoppered, glass vial, 1 in. in diameter and $3 \mathrm{in.} \mathrm{long,} \mathrm{suspended} \mathrm{near} \mathrm{the} \mathrm{specimens.}$ The glycerin dampens out the temperature fluctuations occurring in the test cabinet during a normal operating cycle of the compressor, thus permitting an average temperature to be read and recorded. Recording thermographs are sometimes utilized in the freezing cabinet and the coldroom to obtain continuous records of temperatures. Typical weekly records of coldroom and test cabinet temperatures obtained with thermographs are shown in Figures 8 and 9.

\section{SPECIMEN PREPARATION AND TEST PROCEDURES}

\section{Preparation of Specimens}

Soil specimens for standard frost susceptibility tests are molded in a slightly tapered 6-in.-high steel molding cylinder with a removable base, as shown in Figure 10. The inside diameter is 5.45 inches at the lower end and 5.70 inches at the upper end. The inner surface of the steel cylinder is first lubricated with silicone grease and then lightly coated with paraffin to facilitate ejection of the specimen after molding. Undisturbed field chunk specimens of cohesive soils are prepared for laboratory freezing by trimming them to a uniform diameter and height, both approximately 6 in. The trimmed, undisturbed specimens are placed in vertical-walled containers instead of the insidetapered cylinders.

Two methods are used to mold specimens to desired dry unit weight. Relatively cohesionless, coarse-grained soils, such as sand and sandy gravel, are sometimes prepared by an adaptation of the Providence Vibrated Density Method. ${ }^{8}$ In this method, a predetermined weight of oven-dry soil is placed in the tapered steel cylinder and a load of approximately $1000 \mathrm{lb}$ is applied by a spring loaded piston at the top. The soil within the steel cylinder is then compacted to the required volume by vibrating the cylinder with hammer blows on the side. The soil is then wetted in the cylinder to mobilize internal cohesion before it is removed from the cylinder. Fine-grained soils, such as uniformly very fine sands, silts, glacial tills, and clays, are compacted by tamping them in layers, using the American Association of State Highway Officials (AASHO) test procedure T180-57, ${ }^{2}$ either method "A" or method "D," except that three 2-in. layers are used when stone sizes up to 2 in. in diameter are present in the natural sample. When larger stones are present, they are replaced with an equivalent percentage by weight of smaller stones graded from $1 / 4$ in. to the maximum size utilized in preparing specimens.

Cohesionless soils when prepared by the AASHO procedure are molded at low moisture content; this improves the apparent cohesion and thereby aids specimen handling after molding. For field construction design purposes, cohesive soils are molded at the optimum moisture content and to the desired dry unit weight, using AASHO test procedure T180-57, either method "A" or method "D," depending upon the expected field requirements. For evaluation of the frost potential of materials under existing pavements, subgrade soils obtained from beneath the pavements are tested either in an undisturbed condition or are recompacted in the laboratory to approximate field density (dry unit weight) and moisture conditions.

The molded specimen is removed from the tapered steel molding cylinder by piston pressure applied to the bottom of the specimen and is then fitted snugly into a tapered Lucite freezing cell lined with 1.5-in.-wide, 0.007-in.-thick cellulose acetate strips (or with Teflon). The acetate strips 


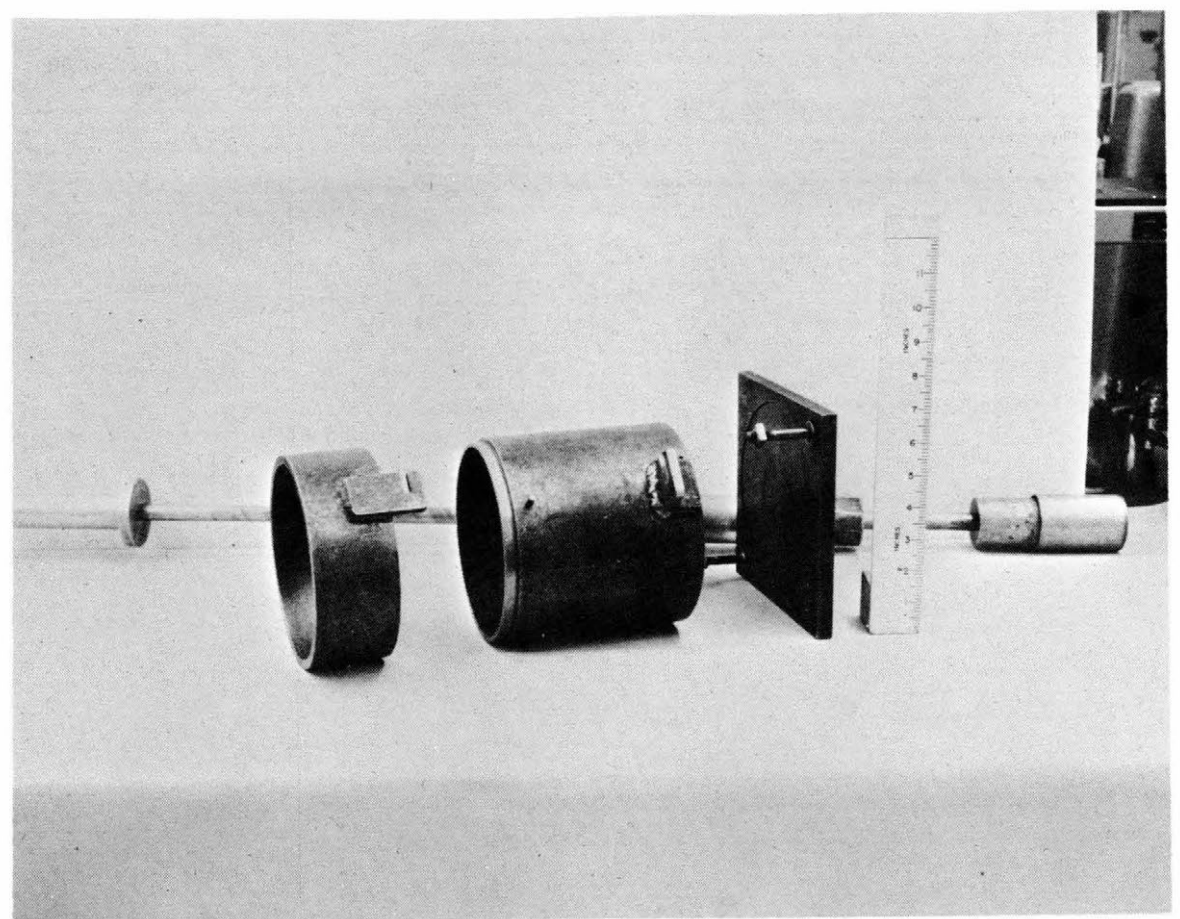

Figure 10. Tapered steel molding cylinder.

are coated on each side with silicone grease and are placed within the cell overlapping each other vertically in a telescopic manner. This is to minimize friction between the specimen and the cell when heave takes place during freezing. Specimens cut from undisturbed field samples are not tapered because of the difficulty of obtaining a uniformly tapered specimen manually. These specimens are free-fitted into straight-walled cylinders of Lucite or waxed, laminated, heavy cardboard, lined with lubricated acetate strips.

\section{Saturation of Specimens}

Prior to freezing, all specimens to be test-frozen in the open system are saturated. Saturation is done in the coldroom at a temperature of approximately $38^{\circ} \mathrm{F}$. Both ends of the Lucite cell containing the soil specimen are covered with filter papers and $3 / 8$-in.-thick flat porous disks and are capped with snug-fitting shallow Lucite caps with nipples extending out from the center for connection of tubing. The air is evacuated from the top of the specimen and the specimen is saturated simultaneously from the bottom with de-aired water (Fig. 6).

\section{Thermocouples in Specimens}

In one of the group of specimens in a cabinet, copper-constantan thermocouples are inserted through the Lucite cell wall at 1-in. intervals along the length of the specimen so that the thermocouple junction is just in contact with the soil except at the top and bottom surfaces where thermocouples are placed closer to the center of the specimen (Fig. 1). The remaining specimens in the group are equipped with thermocouples only on their top and bottom surfaces except for one specimen which is also equipped with thermocouples at 2-in. intervals along its length. The thermocouples placed in the specimen at 1-in. intervals provide an accurate record of the temperature gradient and of the day-by-day advance of freezing temperature into the specimen; the thermocouples in the other specimens provide a double check on the start and completion of the freezing test period. 


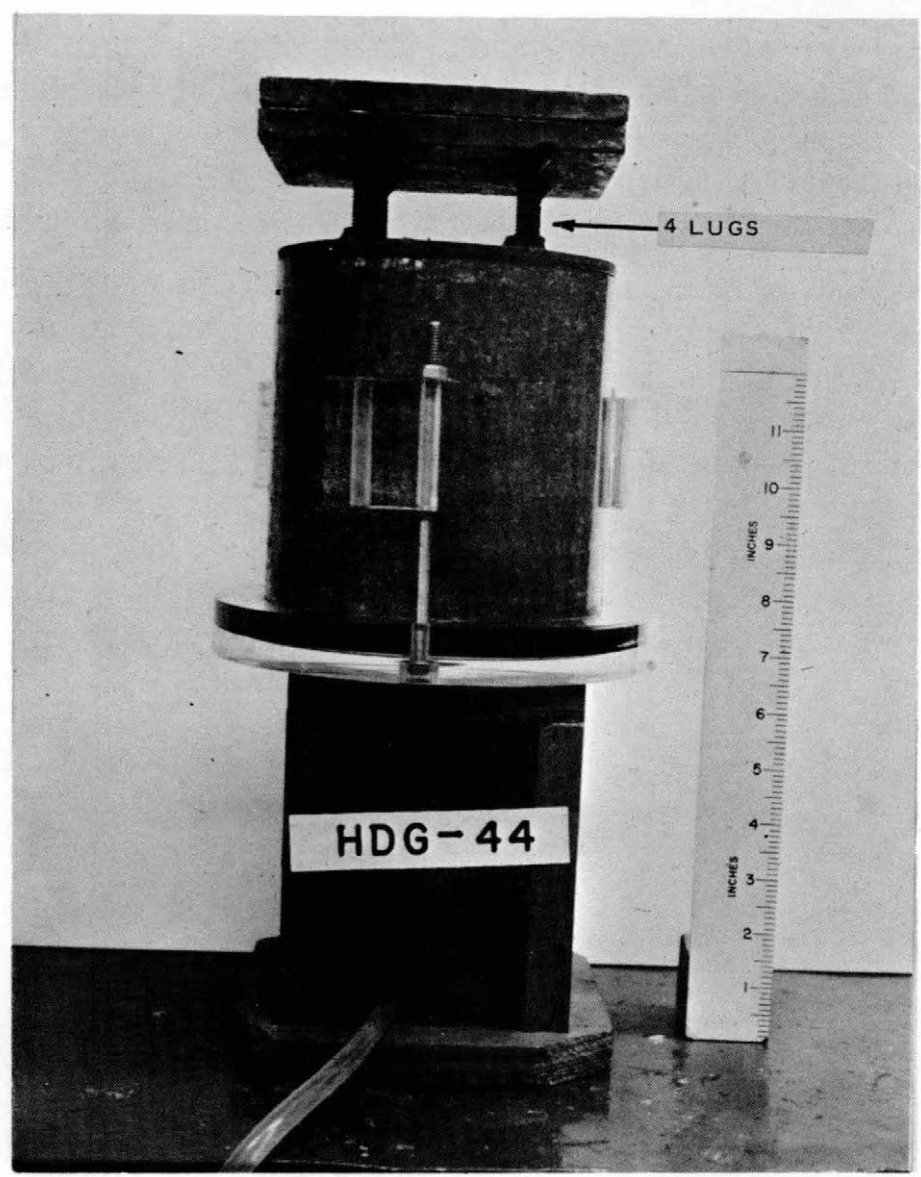

Figure 11. Soil specimen in freezing cell with 0.5 -psi surcharge load.

The thermocouples are inserted through narrow holes drilled through the side of the freezing cell. The entrance points are sealed with a mastic or other suitable sealant. The thermal emf produced by the thermocouples is measured by electrical instruments described in section Description af freezing cabinets and equipment (p. 5). Temperatures are recorded to $0.1^{\circ} \mathrm{F}$.

\section{Load During Freezing}

Unless otherwise stated, all specimens are frozen under a load pressure of $0.5 \mathrm{psi}$ to simulate a minimum field situation consisting of a 6 -in. combined thickness of pavement and base. A steel base plate (1/4-in. thick) is placed on top of the specimen and firmly seated to provide a uniform contact. Four lugs are attached to the base plate to raise the load weights $1 \frac{1}{2}-$ in. so that the air may circulate freely over the base plate (Fig. 11).

\section{Freezing Test Procedure}

Prior to freezing, the specimens are tempered for 18 to 24 hours at $38^{\circ} \mathrm{F}$ in freezing cabinets. A de-aired water supply is connected to the bottom of each specimen or tray through a nipple provided for that purpose. The nipple protrudes through the bottom sheet metal pan and grillwork into the open air space beneath the freezing cabinet which is held at approximately $38^{\circ} \mathrm{F}$, the coldroom 
temperature. The free water level is first raised to the top of the specimen overnight and then lowered, adjusted and maintained at a height of about $1 / 4$ in. above the bottom of the specimen. The top Lucite caps, porous stones and filter papers are removed. The space around the specimens is filled loosely with granulated cork or pellets of polystyrene (Fig. 4) leaving the top surface of the specimens exposed to the cabinet air temperature until freezing is initiated.

Initial freezing of the specimens is obtained by rapidly lowering the air temperature in the freezing cabinet to about $20^{\circ} \mathrm{F}$ or lower, or until the soil surface temperature is lowered to about $30^{\circ} \mathrm{F}$. To ensure crystallization of the soil water, the soil surface is also thinly seeded with fine pulverized ice. When crystallization occurs, the air temperature of the cabinet is raised to $28-30^{\circ} \mathrm{F}$. At this time, thin 6-in.-diam steel base plate and weight(s) (pretempered to about $28^{\circ} \mathrm{F}$ ) are placed on each specimen to provide the 0.5 -psi-load pressure. An alternate method is to place a steel or copper plate with several $3 / 16$-in. perforations over the specimen at the start. Seeding is done by placing pulverized ice into several of the perforations when the soil temperature has been reduced to about $30^{\circ} \mathrm{F}$. The specimens are then gradually frozen from top to bottom by decreasing the cabinet air temperature each day, in decrements, to obtain a rate of penetration of the $32^{\circ} \mathrm{F}$ isotherm of about $1 / 4$ to $1 / 2$-in./day. A slow circulation of air in the cabinet is maintained with the small fan attached to the cover. Cabinet and specimen temperatures and heave measurements are usually recorded twice daily, but at least once as a minimum.

\section{Test Procedure at Completion of Freezing}

Upon completion of freezing, which usually takes 15-20 days, the specimens are removed from the cabinet and freezing cells and are weighed and measured (Fig. 12a). The force required to remove the soil specimen from the freezing cell is noted to assess whether excessive friction may have been present during the test. If the friction value is high, i.e. equivalent to $>2$-psi vertical pressure, a judgment can later be made whether to accept or reject the test results, depending upon the uniformity of the measured heave rate of the specimen. The specimens are split longitudinally into two half sections. One half section (Fig. 12b) is retained for photographing and visual examination of the size and distribution of ice lenses, and the other half section is cut up into 1-in.-thick horizontal segments for determination of water content distribution after freezing.

\section{PRESENTATION OF DATA}

The results of the tests and other information are usually presented as shown in Appendix A. A typical grain size distribution of the material(s) tested is determined after freeze-testing by combined sieve and hydrometer analysis, as shown in Figure 13, and the material is classified according to the Unified Soil Classification System. ${ }^{13}$ The physical properties of the materials, the specimen preparation data and results of freezing tests are given in Tables I and II. The detailed heave data, including the penetration of the $32^{\circ} \mathrm{F}$ temperature plotted versus time, are presented as shown in Figure 14. The water content distribution in a specimen after freezing is presented as shown in Figure 15.

\section{RESULTS}

Under the author's supervision, scores of natural soils from many locations in the United States, as well as Canada, Greenland, Antarctica, Africa and Asia, have been subjected to the type of freezing test described above and have been classified by the author. The results of all tests made on natural soils, using procedures and test conditions described in this report, are summarized in Appendix A. 


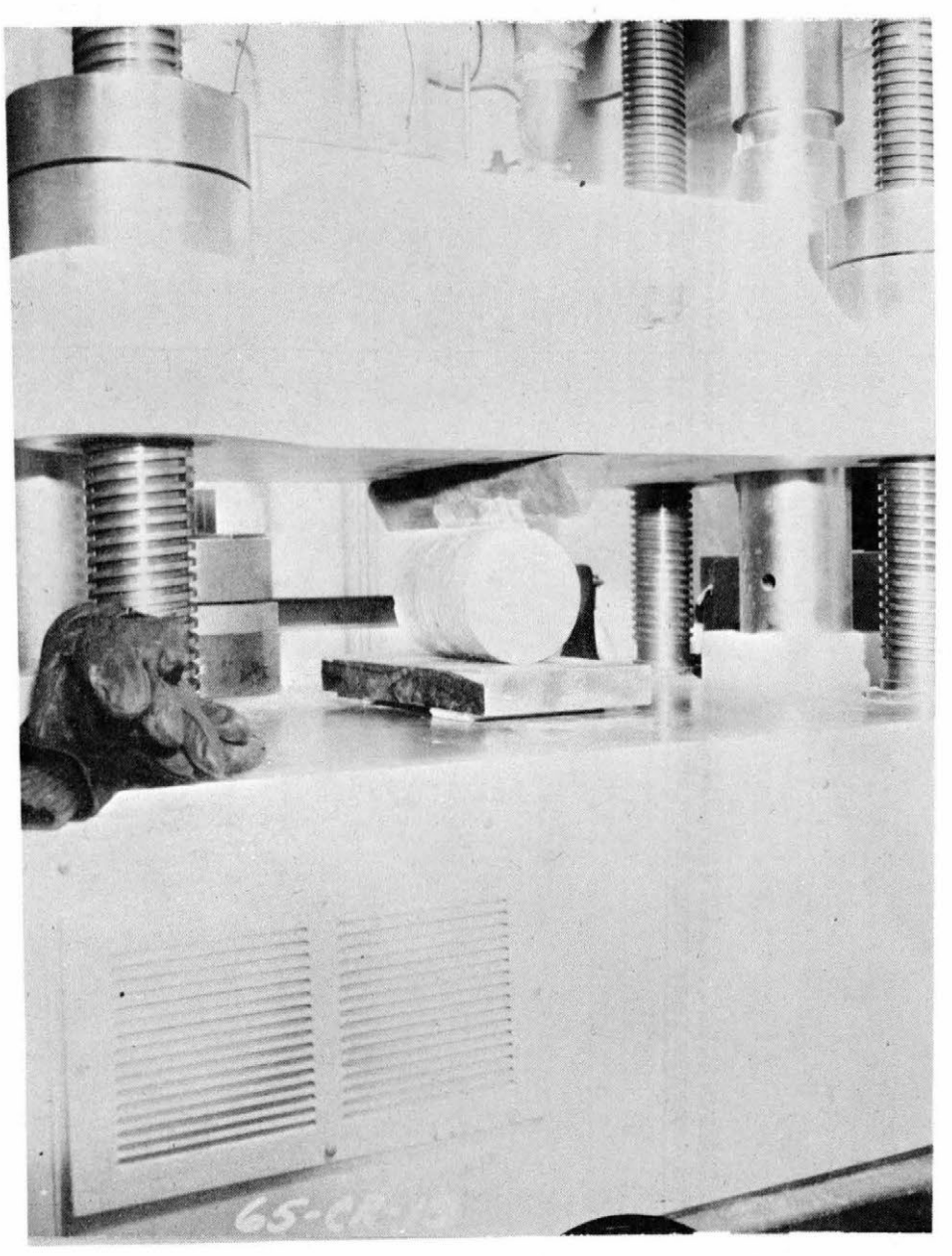

a. Specimen of frozen soil ready for splitting.

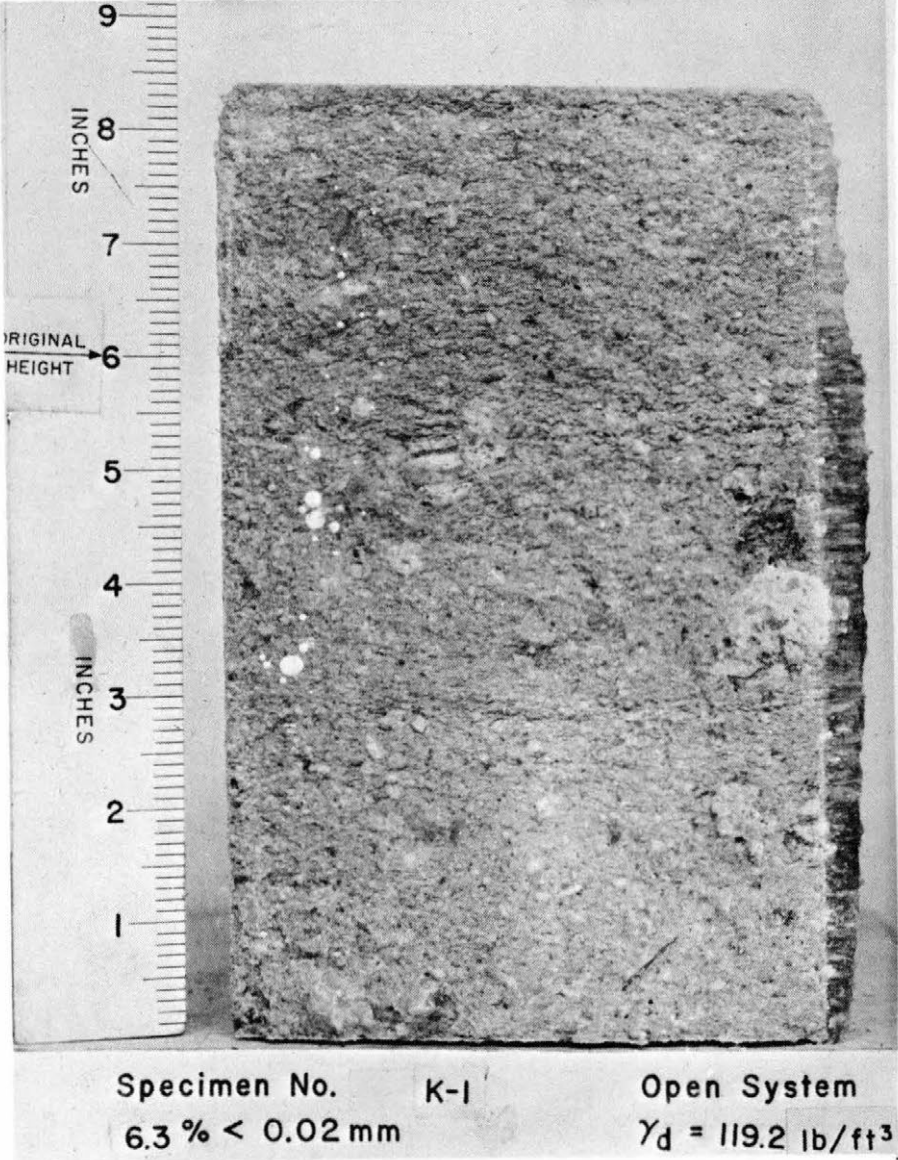

b. Frozen soil specimen after splitting. 


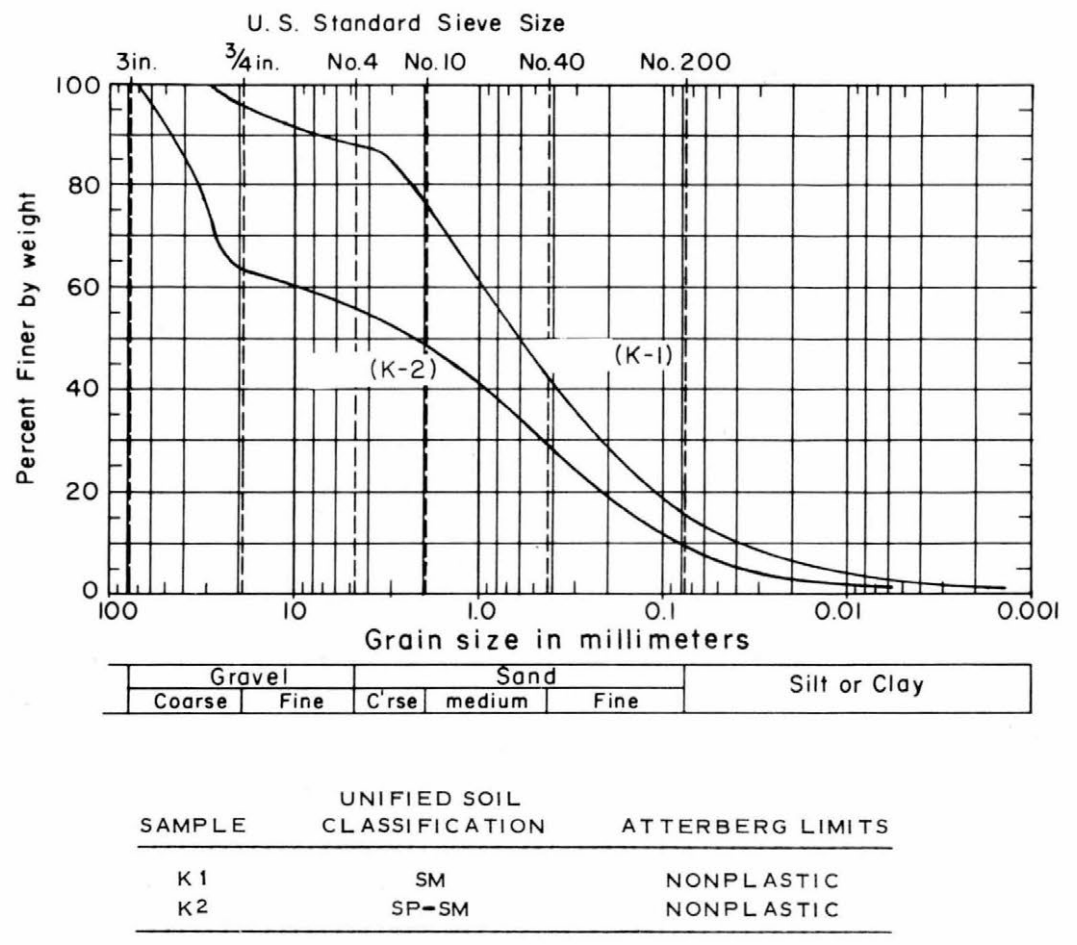

Figure 13. Typical soil gradation curves.

Table I. Typical physical properties of the samples received.

\begin{tabular}{|c|c|c|c|c|c|c|c|c|}
\hline \multirow{2}{*}{$\begin{array}{l}\text { Specimen } \\
\text { no. }\end{array}$} & \multirow{2}{*}{$\begin{array}{l}\text { Percent finer } \\
\text { than } 0.02 \mathrm{~mm} \\
\end{array}$} & \multirow{2}{*}{$\frac{\text { Specific }}{>\# 4}$} & \multirow{2}{*}{$\frac{\text { gravity* }}{<\# 4}$} & \multirow{2}{*}{$\begin{array}{c}\text { Atterberg } \\
\text { limits }\end{array}$} & \multirow{2}{*}{$\begin{array}{c}\text { Max dry } \\
\text { unit weight } \\
\left(1 b / \mathrm{ft}^{3}\right) \\
\end{array}$} & \multirow{2}{*}{$\begin{array}{l}\text { Void } \\
\text { ratio }\end{array}$} & \multicolumn{2}{|c|}{$\begin{array}{c}\text { Unified soil } \\
\text { classification }\end{array}$} \\
\hline & & & & & & & Description & Symbol \\
\hline K 1 & 6.5 & 2.46 & 2.60 & N.P.† & 120.0 & 0.352 & $\begin{array}{l}\text { Slightly gravelly } \\
\text { silty sand }\end{array}$ & SM \\
\hline $\mathrm{K} 2$ & 3.5 & 2.60 & 2.62 & N.P. & 127.0 & 0.240 & $\begin{array}{l}\text { Poorly graded } \\
\text { gravelly silty } \\
\text { sand }\end{array}$ & SP-SM \\
\hline
\end{tabular}

*According to U.S. standard sieve size.

$\dagger$ Nonplastic

Table II. Typical summary of specimen freezing test data.

\begin{tabular}{|c|c|c|c|c|c|c|c|c|c|c|}
\hline \multirow[b]{2}{*}{$\begin{array}{c}\text { Specimen } \\
\text { no. } \\
\end{array}$} & \multirow{2}{*}{$\begin{array}{c}\% \text { Finer } \\
\text { than } \\
0.02 \mathrm{~mm}\end{array}$} & \multirow{2}{*}{$\begin{array}{c}\text { Specimen } \\
\text { dry unit wt } \\
\text { before } \\
\text { fre€zing } \\
\left(\mathrm{lb} / \mathrm{ft}^{3}\right) \\
\end{array}$} & \multirow{2}{*}{$\begin{array}{c}\text { Initial } \\
\text { degree of } \\
\text { saturation } \\
\text { before } \\
\text { freezing } \\
(\%) \\
\end{array}$} & \multicolumn{2}{|c|}{$\begin{array}{c}A_{v g} \\
\text { Water content (\%) }\end{array}$} & \multirow{2}{*}{$\begin{array}{c}\text { Initial } \\
\text { height } \\
\text { before } \\
\text { freezing } \\
\text { (in.) }\end{array}$} & \multirow{2}{*}{$\begin{array}{c}\text { Final } \\
\text { height } \\
\text { after } \\
\text { freezing } \\
\text { (in.) }\end{array}$} & \multirow{2}{*}{$\begin{array}{c}\text { Heave* } \\
(\%)\end{array}$} & \multirow{2}{*}{$\begin{array}{c}\text { Average } \\
\text { rate of } \\
\text { heave } \\
\text { (mm/day) } \\
R \\
\end{array}$} & \multirow{2}{*}{$\begin{array}{c}\text { Max } \\
\text { rate } \\
\text { of } \\
\text { heave }\end{array}$} \\
\hline & & & & $\begin{array}{c}\text { Before } \\
\text { freezing }\end{array}$ & $\begin{array}{c}\text { After } \\
\text { freezing }\end{array}$ & & & & & \\
\hline $\mathrm{K} \cdot 1$ & 6.5 & 119.2 & 96 & 12.5 & 27.2 & 6.0 & 8.37 & 39.5 & 5.5 & 7.5 \\
\hline $\mathrm{K} \cdot 2 \mathrm{~A}$ & 3.5 & 128.3 & 96 & 9.6 & 13.0 & 6.0 & 6.97 & 16.2 & 2.2 & 3.5 \\
\hline $\mathrm{K}-2 \mathrm{~B}$ & 3.5 & 124.5 & 99 & 11.9 & 17.3 & 6.0 & 7.25 & 20.8 & 3.8 & 5.0 \\
\hline Неау & \multicolumn{3}{|c|}{ amount of heave } & & & & & & . & \\
\hline
\end{tabular}



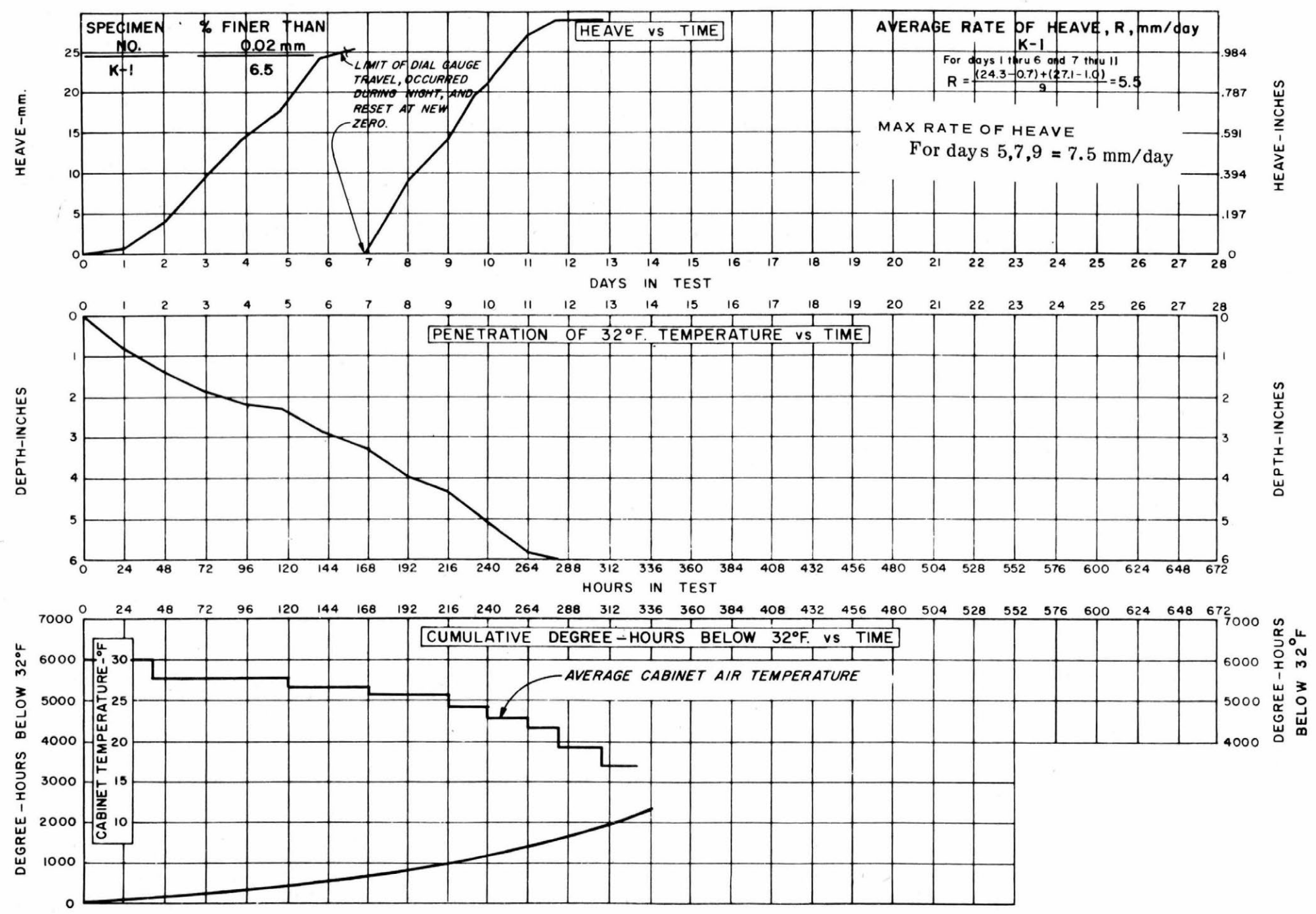

Figure 14. Typical temperature and heave data for specimen K-1 - Open-system test. 


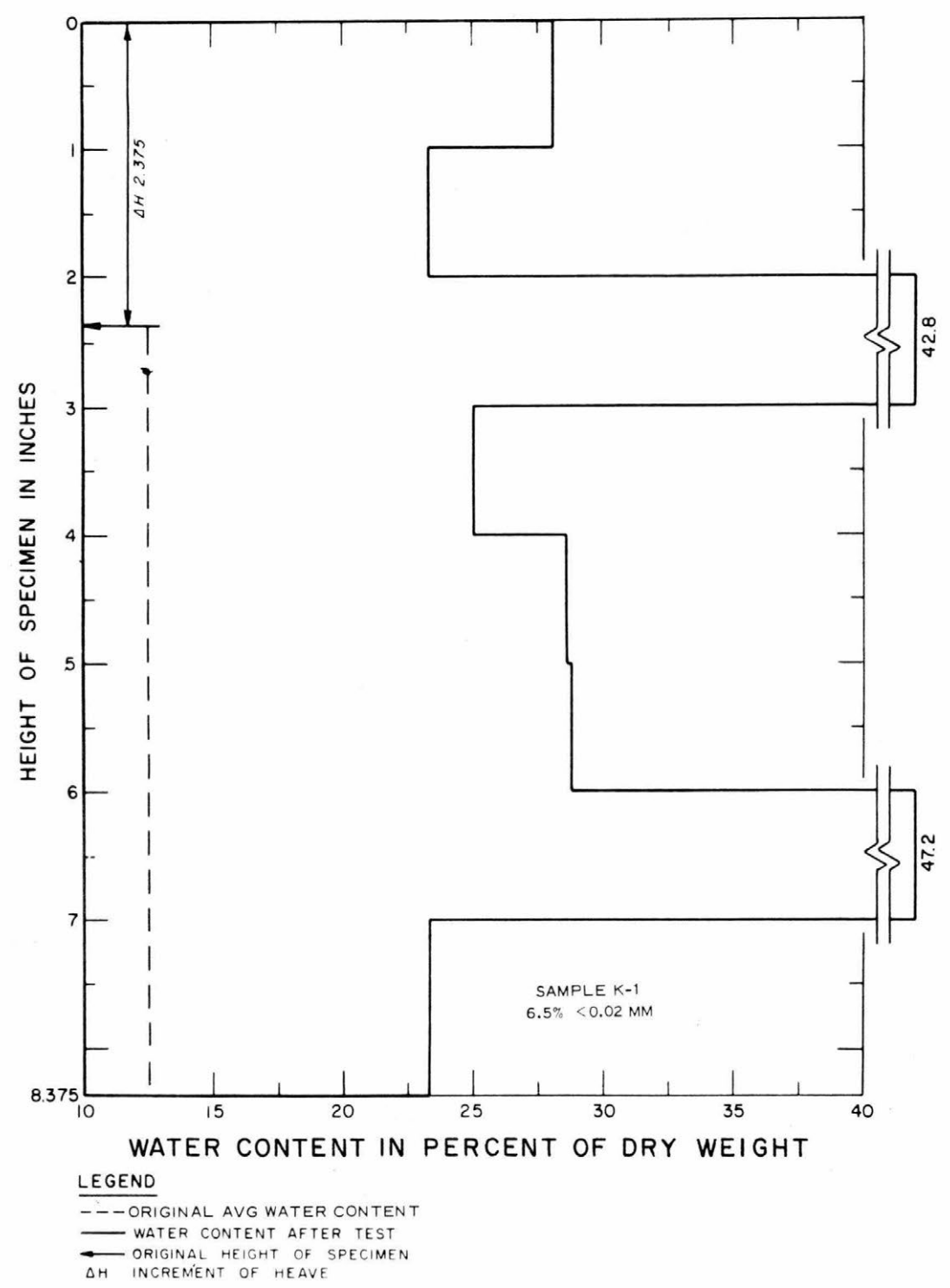

Figure 15. Typical water content vs specimen height.

\section{RECOMMENDATIONS}

It is recommended that experimentation be continued to improve methods and procedures to eliminate friction in freezing tests and to reduce testing time. Because of the wide scatter of some of the test data, it is also recommended that all tests be made in duplicate for increased confidence in the reliability of results.

\section{LITERATURE CITED}

1. American Association of State Highway Officials (AASHO) (1958) Standard method of test for the moisture-density relations of soils using a 5.5-1b rammer and a 12-in. drop. AASHO Designation T99-57. Also American Society for Testing Materials Designation D698-70. 


\section{LITERATURE CITED (Cont'd)}

2. AASHO (1958) Standard method of test for the moisture-density relations of soils using a 10-lb rammer and an 18-in. drop. AASHO Designation T180-57.

3. Beskow, G. (1935) Soil freezing and frost heaving with special application to roads and railroads. The Swedish Geological Society, 26 th Year Book, no. 3, ser. C, no. 275, p. 145. Translated by J.O. Osterberg, The Technological Institute, Northwe stern University, 1947.

4. Casagrande, A. (1932) Discussion of frost heaving. Highway Research Board (HRB) Proceedings, vol. II, part I, p. 168-172.

5. Highway Research Board Committee on Frost Heave and Frost Action in Soils (1955) Frost and permafrost definitions. HRB Bulletin 111, p. 107-110.

6. Hoekstra, P., E. Chamberlain and T. Frate (1965) Frost heaving pressures. HRB Record 101 , p. 28-34.

7. Kaplar, C.W. (1971) Experiments to simplify frost susceptibility testing of soils. U.S. Army Cold Regions Research and Engineering Laboratory (USA CRREL) Technical Report 223 (AD 719237).

8. Lane, Kenneth S. (1948) Providence vibrated density test. Proc. 2nd Int. Conf. of Soil Mech. and Found. Eng., Rotterdam, vol. 4, p. 243-247.

9. Taber, S. (1930) The mechanics of frost heaving. Journal of Geology, vol. 38, no. 4, p. 303-317, May-June.

10. Townsend, D.L. and T.I. Csathy (1963) Soil type in relation to frost action. Ontario Department of Highways, Ontario Joint Highway Research Program Report no. 15 , C.E. Report no. 29.

11. U.S. Army Arctic Construction and Frost Effects Laboratory (USA ACFEL) (1949) Data report of frost investigations, 1943-1949. Frost Effects Laboratory, Corps of Engineers, NED, 3 vol.: vol. I, Summary Report, Special studies, including appendices A-F; vol. II, Field investigations, New England Division; vol. III, Field investigations, Great Lakes Division, Missouri River Division (compiled by C.W. Kaplar).

12. USA ACFEL (1950) Report on cold room and equi pment for frost investigations. Frost Effects Laboratory, Corps of Engineers, ACFEL Bulletin no. 1 (prepared by C.W. Kaplar).

13. U.S. Army Engineer Waterways Experiment Station (1960) The Unified Soil Classification System. Technical Memorandum no. 3-357, vol. 1.

14. Winn, H.F. and P.C. Rutledge (1940) Frost action in highway bases and subgrades. Purdue University Engineering Bulletin, vol. 24, no. 2 (Research ser. no. 73, Highway Research Bulletin no. 4), 104 p. 


\section{APPENDIX A. SUMMARY OF RESULTS OF FREEZING TESTS ON NATURAL SOILS}

\section{INTRODUCTION}

During a number of years when the Frost Effects Laboratory, New England Division, Corps of Engineers, was assigned by the then Airfields and Pavements Branch of the Engineering Division of OCE (now Advanced Technology Branch, Civil Engineering Section, Directorate of Military Construction, OCE) as the official agency where frost susceptibility tests should be run at the request of field offices, scores of soils were submitted for freezing tests. Generally, these were base course materials proposed for use on roads or airfield pavements. Whereas most soils came from construction projects within various parts of the United States, some came from Canada, Greenland, Antarctica, Africa and Asia. In addition to these soils from construction projects, many other fine-grained soils obtained for special studies at ACFEL were tested and are included in the tables in this appendix.

\section{Presentation of Test Data and Results}

Table AI contains test data of soil specimens grouped according to the Unified Soil Classification System. The position of soils within each group is according to the increasing percentage finer by weight than the $0.02-\mathrm{mm}$ size present in the soil. Other data include the physical properties of the material, the results of freezing tests, and the relative frost susceptibility classification as described in page 2 of the main report. Table AI contains the test results on 1) soils that met the test specification of having a dry unit weight of $95 \%$ or greater than that obtained by the appropriate compactive procedure used or specified, and 2) soils that had an initial moisture content before freezing equal to or greater than $85 \%$ of full saturation. The test results listed in Table AI (average rate of heave versus percentage by weight of grains finer than $0.02-\mathrm{mm}$ size) are plotted on Figures A1a-d, in envelopes according to soil type. Table AII contains data grouped similarly in every respect to those in Table AI except that they do not meet the compaction criterion of $95 \%$ or greater and the initial degree of saturation. Table AIII contains heave rate data on specimens tested under a lower load pressure than specimens in Tables AI and AII. Data from Tables AII and AIII have not been plotted in Figures A1a-d. Figures A1 and A2 will be included in the Department of the Army manual on frost design.*

\section{Discussion}

Two heave rates have been computed for each specimen presented in the above tables: an average heave rate and a maximum heave rate, in millimeters per day. This was done to measure the maximum degree of variability, if any, occurring during each test. The degree of variability is expressed as a heave rate variability index. The reason(s) for high variability is not known. It may be reflective of several variables either in some portion of the specimen or in the test controls, such as specimen inhomogeneity (density, layer discontinuities, or other internal influencing factors), cylinder friction, rate of heat extraction, and interruption of water supply (internal and external). A

\footnotetext{
*Department of the Army (1965) Pavement design for frost conditions. Technical Manual TM 5-818-2, July, Currently under revision.
} 
large variability index could be indicative of dominance of several counter forces during tests. Such a test result might be assigned a smaller degree of confidence than one whose test variability index is low.

Recent experimentation at USA CRREL ${ }^{7}$ indicates that some variable degree of friction may exist between the specimen and its container during freezing and heaving. Freezing tests of specimens performed in horizontally segmented (multi-ring) cells usually showed higher heave rate than those of counterpart specimens in inside-tapered solid-walled cells. The inside-tapered cells were a great improvement over straight-walled soil cells. The types of containers used in these tests are indicated in the last column of Tables AI-AIII.

More recent investigations at USA CRREL ${ }^{7}$ to study the development of simplifying and shortening the time interval for the frost susceptibility test revealed that soil specimens in cylinders made of segmented rings 1 in. high usually gave considerably higher heave rates than their counterparts in inside-tapered solid-walled cylinders, especially at the higher rates of frost penetration. Studies to simplify and reduce time for frost susceptibility testing are still in the development and evaluation stage. When sufficient data are available from segmented ring cylinders, it may be possible to correlate these data with the maximum heave rate results in this report.

For each specimen listed in Tables AI-AIII a detailed temperature and heave plot vs time for the complete period of freezing is available in the Laboratory data files. A plot of moisture content distribution with depth after freezing for each inch of specimen height is also available. These plots are similar to those presented in Figures 14 and 15 in the main report. The tabular data presented in this appendix give only the overall initial and final average water content, the percentage of heave, and the rates of heave computed in the manner detailed in the notes contained in the tables.

Figure A2 presents a summary grouping of the individual envelopes shown in Figures A1a-c. It can be seen that there are no distinct, neat groupings, nor is there a unique heave rate for any given percentage of $0.02 \mathrm{~mm}$ in the gradation. The groupings overlap considerably, and it should be noted that the Unified Soil Classification System was not developed for frost classification but is used here because of its wide acceptance in soils engineering. 

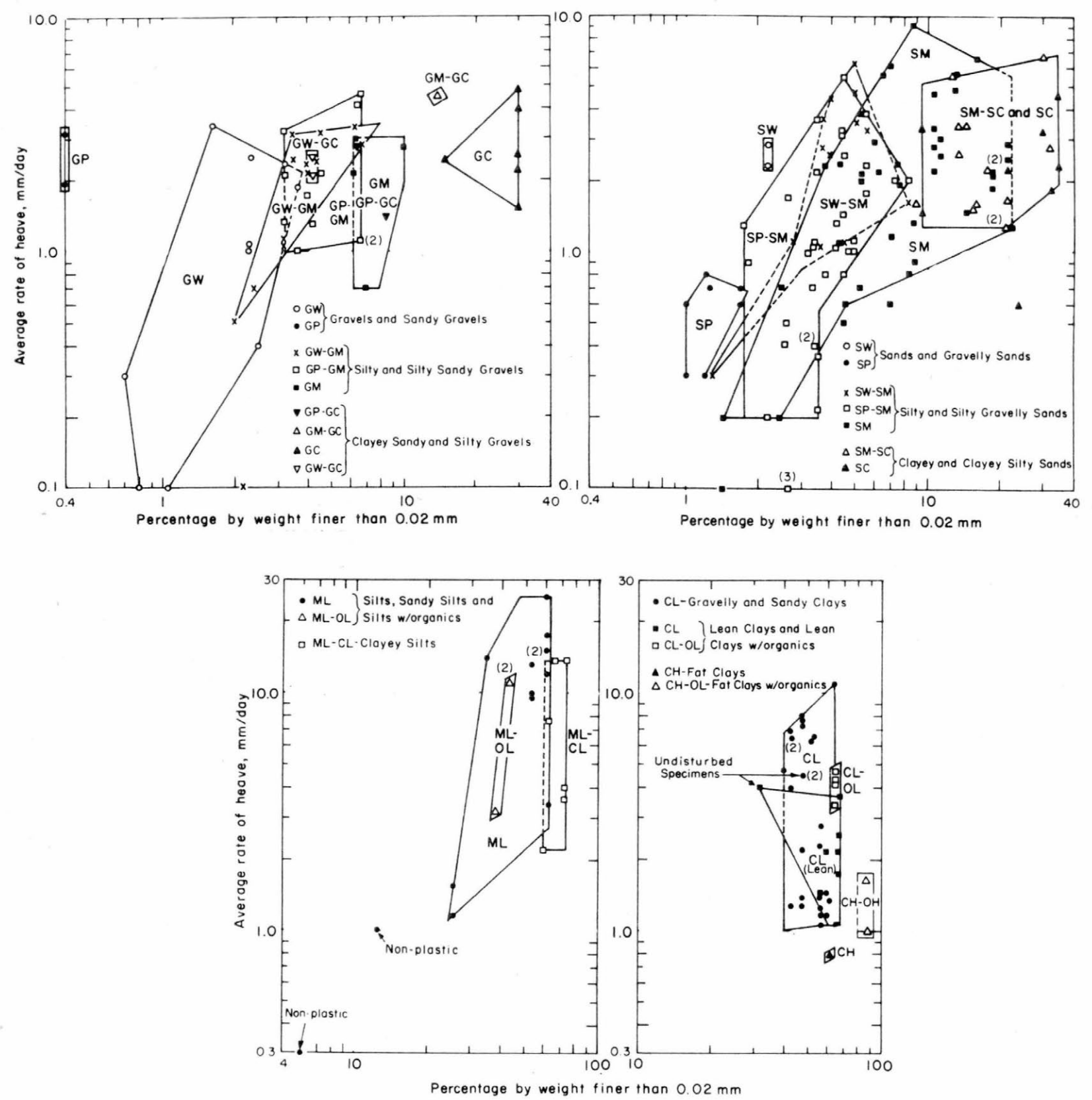

Figure A1. Test results according to soil type. a. Gravels. b. Sands. c. Silts. d. Clays. (2) Indicates two samples of same soil. 


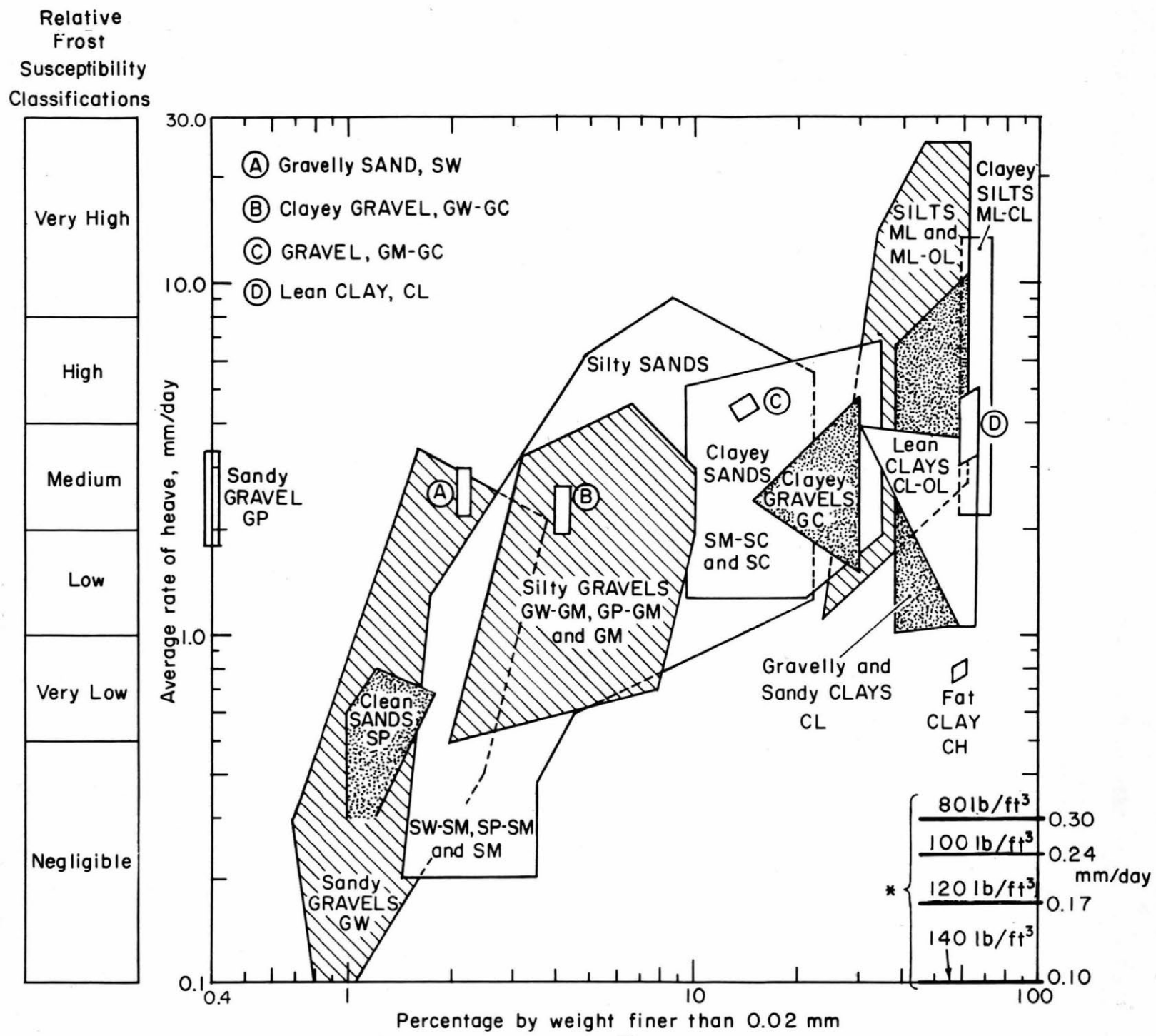

Figure A2. Summary of average rate of heave vs percentage finer than 0.02-mm size for natural soil gradations. 
Table AI. Summary of frost susceptibility tests on natural soils ${ }^{(1)}$ - open svstem nominal load pressure 0.5 psi.

\begin{tabular}{|c|c|c|c|c|c|c|c|c|c|c|c|c|c|c|c|c|c|c|c|c|c|c|c|c|c|c|c|c|c|}
\hline \multirow{3}{*}{$\begin{array}{l}\text { Specimen } \\
\text { Number }\end{array}$} & \multirow{3}{*}{ Moterial Source } & \multicolumn{10}{|c|}{ SOIL GRADATION DATA (As Frozen) } & \multicolumn{5}{|c|}{ PHYSICAL PROPERTIES OF BASIC SOIL } & \multicolumn{5}{|c|}{ SPECIMEN DATA (AS Molded) } & \multicolumn{7}{|c|}{ FREEZING TEST DATA } & \multirow{3}{*}{$\begin{array}{l}\text { Typo } \\
\text { of } \\
\text { Cyl. } \\
\text { (12) }\end{array}$} \\
\hline & & \multirow{2}{*}{$\begin{array}{l}\text { Unified } \\
\text { Soil } \\
\text { Clossifi- } \\
\text { cotion } \\
\text { Symbol } \\
\text { (2) }\end{array}$} & \multirow{2}{*}{$\begin{array}{c}\text { Maxi- } \\
\text { mum } \\
\text { Size } \\
\\
\text { in. }\end{array}$} & \multicolumn{6}{|c|}{ Percent finer, mm } & \multicolumn{2}{|c|}{$\begin{array}{c}\text { Coefficients } \\
\text { (3) }\end{array}$} & \multicolumn{2}{|c|}{$\begin{array}{l}\text { Atterberg } \\
\text { Limits (4) }\end{array}$} & \multirow{2}{*}{$\begin{array}{l}\text { Specific } \\
\text { Grovity }\end{array}$} & \multicolumn{2}{|c|}{$\begin{array}{c}\text { Compaction Dato } \\
\text { (5) }\end{array}$} & \multirow{2}{*}{$\begin{array}{c}\text { Dry } \\
\text { Unit } \\
\text { Weight } \\
\text { pof }\end{array}$} & \multirow{2}{*}{$\begin{array}{c}\text { Degree } \\
\text { of } \\
\text { Compac- } \\
\text { tion } \\
\% \\
\%\end{array}$} & \multirow{2}{*}{$\begin{array}{l}\text { Void } \\
\text { Ratio }\end{array}$} & \multirow{2}{*}{$\begin{array}{l}\text { G, of } \\
\text { stort } \\
\text { of } \\
\text { Test } \\
(6) \\
\%\end{array}$} & \multirow{2}{*}{\begin{tabular}{|c|} 
Perme- \\
obilify \\
$k$ \\
$(7)$ \\
$\mathrm{cm} / \mathrm{sec}$ \\
$\times 10^{-4}$
\end{tabular}} & \multicolumn{2}{|c|}{$\begin{array}{c}\text { Avg. } \\
\text { worter } \\
\text { Content }\end{array}$} & \multirow{2}{*}{\begin{tabular}{|c|} 
\\
Totol \\
Heavo \\
(8) \\
$\%$ \\
\end{tabular}} & \multicolumn{2}{|c|}{\begin{tabular}{|c|}
$\begin{array}{l}\text { Rate of } \\
\text { Heove } \\
\text { mm/day (9) }\end{array}$ \\
\end{tabular}} & \multirow{2}{*}{$\begin{array}{l}\text { Heave } \\
\text { Hate } \\
\text { Var. } \\
\text { Index } \\
(10)\end{array}$} & \multirow{2}{*}{\begin{tabular}{|c|} 
Frost \\
Susc \\
Closs \\
(III)
\end{tabular}} & \\
\hline & & & & 4.76 & 0.42 & 0.074 & 0.02 & 0.01 & 0.005 & $5 c_{u}$ & $c_{c}$ & LL & PI & & $\left|\begin{array}{c}\text { Maximum } \\
\text { Ory Unit } \\
\text { Weight } \\
\text { pcf }\end{array}\right| \begin{array}{c}0 \\
\text { pct }\end{array}$ & $\begin{array}{c}\text { Optimum } \\
\text { Moisture } \\
\text { Content } \\
\% \\
\end{array}$ & & & & & & \begin{tabular}{|c|} 
Before \\
Test \\
$\%$
\end{tabular} & \begin{tabular}{|l} 
After \\
Tost \\
$\%$
\end{tabular} & & Avg. & Max. & & & \\
\hline $\begin{array}{l}\mathrm{BPR}-5 \\
\mathrm{KA}-4 \\
\mathrm{FC}-4 \\
\mathrm{PBJ}-6 \\
\mathrm{DFB}-2\end{array}$ & $\begin{array}{l}\text { B.P.R. Alaska } \\
\text { Keflavlk } \\
\text { Fairehild } \\
\text { Project Blue Joy } \\
\text { Dow Fleld }\end{array}$ & GW & $\begin{array}{l}1 \\
3 / 4 \\
12 \\
3 / 4 \\
3 / 4\end{array}$ & $\begin{array}{l}40 \\
49 \\
30 \\
38 \\
49\end{array}$ & $\begin{array}{l}5.0 \\
10 \\
6.0 \\
28 \\
12\end{array}$ & $\begin{array}{l}1.5 \\
3.0 \\
2.9 \\
4.0 \\
4.7\end{array}$ & $\begin{array}{l}0.7 \\
0.8 \\
1.1 \\
1.7 \\
2.4\end{array}$ & $\begin{array}{l}0.4 \\
0.8 \\
0.7 \\
1.3 \\
1.7\end{array}$ & $\begin{array}{l}0.2 \\
0.5 \\
0.4 \\
0.9 \\
0.9\end{array}$ & \begin{tabular}{|l|}
114 \\
17 \\
8.2 \\
53 \\
20
\end{tabular} & $\begin{array}{l}1.0 \\
1.4 \\
1.7 \\
2.4 \\
1.1\end{array}$ & $\frac{\mathrm{GRAV} \mathrm{V}_{\mathrm{i}}}{\mathrm{a}}$ & ELS AN & $\begin{array}{l}2.77 \\
2.81 \\
2.96 \\
2.72 \\
2.72\end{array}$ & $\begin{array}{l}126.7 \text { (b) } \\
112.0 \text { (b) } \\
128.8 \text { (b) } \\
1148.2 \text { (b) } \\
11.2 .9 \text { (b) }\end{array}$ & $\begin{array}{l}\overline{-} \\
\overline{-} \\
z\end{array}$ & $\begin{array}{l}124 \\
109 \\
126 \\
1140 \\
138\end{array}$ & $\begin{array}{l}98 \\
98 \\
98 \\
95 \\
97\end{array}$ & $\begin{array}{l}0.395 \\
0.589 \\
0.462 \\
0.212 \\
0.231\end{array}$ & $\begin{array}{r}90 \\
100 \\
100 \\
100 \\
100\end{array}$ & $\begin{array}{l}\bar{z} \\
\bar{z}\end{array}$ & $\begin{array}{r}13.4 \\
21.3 \\
11.7 \\
7.8 \\
8.5\end{array}$ & $\begin{array}{r}9.8 \\
17.7 \\
10.7 \\
28.4 \\
24.8\end{array}$ & $\begin{array}{r}4.1 \\
5.9 \\
1.3 \\
51.8 \\
52.5\end{array}$ & $\begin{array}{l}0.3 \\
0.1 \\
0.1 \\
3.4 \\
2.6\end{array}$ & \begin{tabular}{l|}
0.8 \\
0.3 \\
0.3 \\
5.8 \\
4.3
\end{tabular} & $\begin{array}{l}2.67 \\
3.00 \\
3.00 \\
1.70 \\
1.65\end{array}$ & $\mid$\begin{tabular}{l|}
$N-\sqrt{L}$ \\
$N$ \\
$N$ \\
$M-H$ \\
$M-H$
\end{tabular} & $\begin{array}{l}\text { SC } \\
\text { SC } \\
\text { TL } \\
\text { SC } \\
\text { SC }\end{array}$ \\
\hline $\begin{array}{l}\mathrm{DFB} \propto 3 \\
\mathrm{DFB}-4 \\
\mathrm{HN}-1 \\
\mathrm{HN}-2 \\
\mathrm{LSG}-7 \\
\mathrm{LSG}-36\end{array}$ & $\begin{array}{l}\text { Dow Field } \\
\text { Dow Field } \\
\text { Hancock } \\
\text { Hancock } \\
\text { Loring } \\
\text { Loring }\end{array}$ & & $\begin{array}{l}3 / 4 \\
3 / 4 \\
3 / 4 \\
3 / 4 \\
3 / 4 \\
2\end{array}$ & $\begin{array}{l}42 \\
42 \\
35 \\
35 \\
39 \\
40\end{array}$ & \begin{tabular}{|c|}
13 \\
13 \\
7.0 \\
7.0 \\
71 \\
8.0
\end{tabular} & $\begin{array}{l}4.9 \\
4.9 \\
4.8 \\
4.8 \\
4.9 \\
4.6\end{array}$ & $\begin{array}{l}2.4 \\
2.4 \\
2.6 \\
2.6 \\
3.2 \\
3.7\end{array}$ & \begin{tabular}{c|}
- \\
1.5 \\
1.5 \\
2.6 \\
3.3
\end{tabular} & $\begin{array}{c}- \\
1.0 \\
1.0 \\
2.0 \\
2.7\end{array}$ & $\begin{array}{c}33 \\
33 \\
8.2 \\
8.2 \\
24 \\
17\end{array}$ & \begin{tabular}{|l|}
2.4 \\
2.4 \\
1.8 \\
1.8 \\
1.4 \\
1.0
\end{tabular} & $\begin{array}{l}18 \\
18\end{array}$ & $\begin{array}{l}3.0 \\
3.0\end{array}$ & $\begin{array}{l}2.73 \\
2.73 \\
2.76 \\
2.76 \\
2.71 \\
2.71\end{array}$ & $\begin{array}{l}137.6 \text { (b) } \\
137.6 \text { (b) } \\
136.0 \text { (b) } \\
136.0 \text { (b) } \\
143.8 \text { (d) } \\
139.3 \text { (b) }\end{array}$ & $\begin{array}{l}= \\
= \\
=\end{array}$ & $\begin{array}{l}131 \\
131 \\
130 \\
132 \\
137 \\
135\end{array}$ & $\begin{array}{l}95 \\
95 \\
96 \\
97 \\
95 \\
97\end{array}$ & \begin{tabular}{|l|}
0.296 \\
0.300 \\
0.322 \\
0.309 \\
0.237 \\
0.255
\end{tabular} & $\begin{array}{r}95 \\
99 \\
100 \\
100 \\
100 \\
100\end{array}$ & $\begin{array}{l}\overline{-} \\
\bar{z} \\
\bar{z} .05\end{array}$ & $\begin{array}{r}10.3 \\
10.9 \\
11.6 \\
21.2 \\
8.6 \\
9.4\end{array}$ & $\begin{array}{l}13.6 \\
14.8 \\
11.8 \\
12.3 \\
12.8 \\
17.7\end{array}$ & $\begin{array}{l}13.8 \\
15.7 \\
12.8 \\
12.8 \\
18.3 \\
24.6\end{array}$ & $\begin{array}{l}1.0 \\
1.1 \\
0.7 \\
0.4 \\
2.3 \\
1.9\end{array}$ & $\begin{array}{l}1.6 \\
1.8 \\
1.3 \\
1.5 \\
3.2 \\
3.2\end{array}$ & $\begin{array}{l}1.60 \\
1.64 \\
1.86 \\
3.75 \\
1.39 \\
1.68\end{array}$ & \begin{tabular}{|l|}
$L$ \\
$L$ \\
$V L-L$ \\
$N-L$ \\
$M$ \\
$L-M$
\end{tabular} & $\begin{array}{l}\mathrm{SC} \\
\mathrm{SC} \\
\mathrm{SC} \\
\mathrm{SC} \\
\mathrm{SC} \\
\mathrm{SC}\end{array}$ \\
\hline $\begin{array}{l}\text { PBJ-11 } \\
\text { PEJ-12 }\end{array}$ & $\begin{array}{l}\text { Project Blue Jay } \\
\text { Project BIve Jay }\end{array}$ & GP & $\begin{array}{r}3 / 4 \\
3 / 4 \\
3 / 4\end{array}$ & $\begin{array}{l}46 \\
46\end{array}$ & $\begin{array}{l}17 \\
17\end{array}$ & $\begin{array}{l}1.4 \\
1.4\end{array}$ & $\begin{array}{l}0.4 \\
0.4\end{array}$ & $\begin{array}{l}0.3 \\
0.3 \\
\end{array}$ & $\begin{array}{l}0.2 \\
0.2\end{array}$ & $\begin{array}{l}57 \\
57 \\
\end{array}$ & $\begin{array}{l}0.4 \\
0.4 \\
\end{array}$ & & & $\begin{array}{l}2.74 \\
2.74 \\
\end{array}$ & $\begin{array}{ll}148.2 & (b) \\
\mu_{1} 8.2 & \text { (b) }\end{array}$ & $=$ & $\frac{114}{1140}$ & $\begin{array}{l}97 \\
95\end{array}$ & $\begin{array}{l}0.188 \\
0.218\end{array}$ & $\begin{array}{r}100 \\
91 \\
\end{array}$ & $\begin{array}{l}0.111 \\
0.21 \\
\end{array}$ & $\begin{array}{l}6.9 \\
7.3 \\
\end{array}$ & $\begin{array}{l}12.4 \\
25.5 \\
\end{array}$ & $\begin{array}{l}16.0 \\
43.0 \\
\end{array}$ & $\begin{array}{l}1.9 \\
3.1\end{array}$ & $\begin{array}{l}3.3 \\
5.7 \\
\end{array}$ & 1.74 & $\underset{M}{L}-M$ & $\begin{array}{l}\mathrm{sc} \\
\mathrm{sc}\end{array}$ \\
\hline $\begin{array}{l}\text { CDB-1 } \\
\text { KA-8 } \\
\text { KA-9 } \\
\text { TAFB-1 } \\
\text { TAFB-3 }\end{array}$ & $\begin{array}{l}\text { Cape Dyer } \\
\text { Keflavik } \\
\text { Keflavik } \\
\text { Thule } \\
\text { Thule }\end{array}$ & GW-GM & $\begin{array}{l}2 / 4 \\
3 / 4 \\
3 / 4 \\
3 / 4 \\
3 / 4\end{array}$ & $\begin{array}{l}42 \\
42 \\
42 \\
42 \\
44\end{array}$ & $\begin{array}{l}\frac{19}{34} \\
\frac{14}{4} \\
18 \\
18\end{array}$ & $\begin{array}{l}5.7 \\
5.3 \\
5.3 \\
7.0 \\
7.0\end{array}$ & $\begin{array}{l}2.0 \\
2.1 \\
2.1 \\
2.5 \\
2.9\end{array}$ & $\begin{array}{l}1.3 \\
1.2 \\
1.2 \\
1.9 \\
2.1\end{array}$ & $\begin{array}{l}1.0 \\
0.7 \\
0.7 \\
1.3 \\
1.5\end{array}$ & $\begin{array}{l}87 \\
38 \\
38 \\
59 \\
57\end{array}$ & $\begin{array}{l}1.1 \\
2.2 \\
2.2 \\
1.7 \\
2.0\end{array}$ & $\begin{array}{ll} & \\
17.8 \\
17.8\end{array}$ & $\begin{array}{l}2.4 \\
2.4 \\
\end{array}$ & \begin{tabular}{|l|} 
SANDY GRA \\
2.67 \\
2.77 \\
2.77 \\
2.76 \\
2.77
\end{tabular} & $\begin{array}{ll}-140.8 & \text { (b) } \\
123.0 & (\mathrm{~b}) \\
123.0 & \text { (b) } \\
\mu_{4} 3.6 & \text { (b) } \\
243.6 & \text { (b) }\end{array}$ & $\begin{array}{l}z \\
z\end{array}$ & & $\begin{array}{l}99 \\
97 \\
98 \\
98 \\
98\end{array}$ & $\begin{array}{l}0.200 \\
0.446 \\
0.435 \\
0.228 \\
0.230\end{array}$ & $\begin{array}{r}100 \\
91 \\
85 \\
100 \\
100\end{array}$ & $\begin{array}{l}\bar{z} \\
\bar{z}\end{array}$ & $\begin{array}{r}7.5 \\
11.6 \\
13.3 \\
8.3 \\
8.4\end{array}$ & $\begin{array}{l}10.8 \\
15.0 \\
14.8 \\
13.0 \\
16.2\end{array}$ & $\begin{array}{r}9.8 \\
1.3 \\
2.1 \\
13.5 \\
21.4\end{array}$ & $\begin{array}{l}0.5 \\
0.1 \\
0.1 \\
0.7 \\
1.2\end{array}$ & $\begin{array}{l}1.0 \\
0.2 \\
0.3 \\
1.5 \\
2.5\end{array}$ & $\begin{array}{l}2.00 \\
2.00 \\
3.00 \\
2.14 \\
2.08\end{array}$ & \begin{tabular}{|l}
$\nabla L$ \\
$N$ \\
$N$ \\
$N$ \\
$L-M$ \\
$L-M$
\end{tabular} & $\begin{array}{l}\mathrm{T} \\
\mathrm{SC} \\
\mathrm{SC} \\
\mathrm{SC} \\
\mathrm{SC}\end{array}$ \\
\hline $\begin{array}{l}\text { DFSB-2 } \\
\text { DFSB-3 } \\
\text { SA-1 } \\
\text { SA-5 } \\
\text { LSG-8 }\end{array}$ & $\begin{array}{l}\text { Dow Field } \\
\text { Dow Field } \\
\text { Stewart } \\
\text { Stewart } \\
\text { Loring }\end{array}$ & & $\begin{array}{c}3 / 4 \\
3 / 4 \\
2 \\
2 \\
3 / 4\end{array}$ & $\begin{array}{l}49 \\
49 \\
53 \\
53 \\
51\end{array}$ & $\begin{array}{l}17 \\
17 \\
20 \\
20 \\
12\end{array}$ & $\begin{array}{l}8.0 \\
8.0 \\
7.4 \\
7.4 \\
5.5\end{array}$ & $\begin{array}{l}3.2 \\
3.2 \\
3.5 \\
3.5 \\
4.0\end{array}$ & $\begin{array}{c}- \\
- \\
2.5 \\
2.5 \\
3.3\end{array}$ & $\begin{array}{l}- \\
- \\
1.3 \\
1.3 \\
2.3\end{array}$ & $\left\{\begin{array}{l}57 \\
57 \\
48 \\
48 \\
22\end{array}\right.$ & $\begin{array}{l}2.1 \\
2.1 \\
1.0 \\
1.0 \\
1.3\end{array}$ & & & $\begin{array}{l}2.73 \\
2.73 \\
2.69 \\
2.69 \\
2.71\end{array}$ & 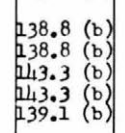 & $\begin{array}{l}\bar{z} \\
\bar{z}\end{array}$ & $\begin{array}{l}134 \\
132 \\
139 \\
11,1 \\
137\end{array}$ & $\begin{array}{l}96 \\
95 \\
97 \\
98 \\
98\end{array}$ & $\begin{array}{l}0.274 \\
0.288 \\
0.231 \\
0.222 \\
0.237\end{array}$ & $\begin{array}{r}100 \\
99 \\
100 \\
100 \\
98\end{array}$ & $\begin{array}{l}4.6 \\
6.2 \\
2.3 \\
2.0 \\
1.1\end{array}$ & $\begin{array}{r}10.0 \\
10.4 \\
8.4 \\
8.1 \\
8.4\end{array}$ & $\begin{array}{l}16.8 \\
15.9 \\
13.7 \\
19.1 \\
13.2\end{array}$ & $\begin{array}{l}20.5 \\
18.4 \\
16.2 \\
29.6 \\
14.6\end{array}$ & $\begin{array}{l}1.1 \\
1.2 \\
3.1 \\
2.5 \\
2.1\end{array}$ & \begin{tabular}{|l|}
1.4 \\
1.6 \\
3.7 \\
4.0 \\
2.7
\end{tabular} & $\begin{array}{l}1.27 \\
1.33 \\
1.19 \\
1.00 \\
1.28\end{array}$ & $\begin{array}{l}\mathrm{L} \\
\mathrm{L} \\
\mathrm{L} \\
\mathrm{M} \\
\mathrm{M} \\
\mathrm{M}\end{array}$ & $\begin{array}{l}\text { SC } \\
\text { SC } \\
T \\
T \\
\text { SC }\end{array}$ \\
\hline $\begin{array}{l}\text { AFG-11 } \\
\text { BPG-1 } \\
\text { PI-1 } \\
\text { LSG-37 } \\
\text { LSG } 141 \\
\text { LSG-1A }\end{array}$ & $\begin{array}{l}\text { Afghantstan } \\
\text { Bowley Pit } \\
\text { Preaque Isle } \\
\text { Loring } \\
\text { Loring } \\
\text { Loring }\end{array}$ & & $\begin{array}{c}1 \\
3 \\
3 / 4 \\
1 \\
1 \\
3 / 4\end{array}$ & $\begin{array}{l}52 \\
47 \\
44 \\
48 \\
50 \\
47\end{array}$ & $\begin{array}{l}24 \\
23 \\
23 \\
94 \\
9.0 \\
22 \\
17\end{array}$ & $\begin{array}{l}9.2 \\
7.5 \\
7.0 \\
5.6 \\
8.0 \\
9.5\end{array}$ & $\begin{array}{l}4.0 \\
4.3 \\
4.5 \\
4.6 \\
6.3 \\
6.8\end{array}$ & $\begin{array}{l}3.0 \\
3.2 \\
3.1 \\
4.1 \\
5.4 \\
-\end{array}$ & $\begin{array}{l}2.2 \\
1.8 \\
2.5 \\
3.1 \\
4.0 \\
-\end{array}$ & $\begin{array}{l}47 \\
32 \\
16 \\
26 \\
-\end{array}$ & $\begin{array}{l}- \\
2.2 \\
1.3 \\
1.0 \\
1.9 \\
-\end{array}$ & $\begin{array}{l}16.8 \\
24 \\
17\end{array}$ & $\begin{array}{l}4.7 \\
6 \\
17\end{array}$ & $\begin{array}{l}2.73 \\
2.69 \\
2.74 \\
2.71 \\
2.71 \\
2.71\end{array}$ & $\begin{array}{c}143.2 \text { (b) } \\
138.0 \text { (b) } \\
1312.9 \text { (b) } \\
138.4 \text { (b) } \\
--\end{array}$ & $\begin{array}{l}\bar{z} \\
\bar{z} \\
z\end{array}$ & $\begin{array}{l}1112 \\
132 \\
1140 \\
134 \\
134 \\
137\end{array}$ & $\begin{array}{r}9 \overline{96} \\
98 \\
97 \\
>95 \\
-\end{array}$ & $\begin{array}{l}0.202 \\
0.267 \\
0.220 \\
0.259 \\
0.263 \\
0.250\end{array}$ & $\begin{array}{r}99 \\
100 \\
100 \\
100 \\
96 \\
100\end{array}$ & $\begin{array}{c}= \\
\overline{-} \\
=\end{array}$ & $\begin{array}{l}7.4 \\
9.4 \\
8.1 \\
9.6 \\
9.3 \\
9.1\end{array}$ & $\begin{array}{l}23 . \\
16.8 \\
21.1 \\
19.1 \\
30.0\end{array}$ & $\begin{array}{l}25.0 \\
38.1 \\
22.6 \\
34.3 \\
32.8 \\
61.1\end{array}$ & $\begin{array}{r}2.3 \\
2.5 \\
2.0 \\
3.1 \\
3.4 \\
2.9\end{array}$ & $\begin{array}{l}3.7 \\
3.5 \\
2.2 \\
5.0 \\
4.3 \\
4.5\end{array}$ & $\begin{array}{l}1.60 \\
1.40 \\
1.10 \\
1.61 \\
1.26 \\
1.55\end{array}$ & $\begin{array}{l}M \\
M \\
M \\
M-H \\
M-H \\
M-H \\
M-B\end{array}$ & $\begin{array}{l}\mathrm{T} \\
\mathrm{T} \\
\mathrm{SC} \\
\mathrm{SC} \\
\mathrm{SC} \\
\mathrm{SC}\end{array}$ \\
\hline $\begin{array}{l}\text { CDB-2 } \\
\text { SA-3 } \\
\text { SA-7 } \\
\text { MP-3 } \\
\text { PBJ-13 }\end{array}$ & $\begin{array}{l}\text { Cape Dyer } \\
\text { Stewart } \\
\text { Stewart } \\
\text { Marble Point } \\
\text { Project Blue Jay }\end{array}$ & GP-GM & $\begin{array}{c}2 \\
2 \\
2 \\
2 \\
3 / 4\end{array}$ & $\begin{array}{l}47 \\
51 \\
51 \\
56 \\
54\end{array}$ & $\begin{array}{l}23 \\
12 \\
12 \\
32 \\
32\end{array}$ & $\begin{array}{l}9.1 \\
5.8 \\
5.8 \\
11 \\
10\end{array}$ & $\begin{array}{l}3.2 \\
3.3 \\
3.3 \\
3.7 \\
4.0\end{array}$ & $\begin{array}{l}2.1 \\
2.5 \\
2.5 \\
3.0 \\
2.2\end{array}$ & $\begin{array}{l}1.5 \\
1.8 \\
1.8 \\
2.0 \\
1.5\end{array}$ & \begin{tabular}{|l}
120 \\
23 \\
23 \\
101 \\
81
\end{tabular} & $\begin{array}{l}0.6 \\
0.8 \\
0.8 \\
0.3 \\
0.4\end{array}$ & & & $\begin{array}{l}2.69 \\
2.70 \\
2.70 \\
2.74 \\
2.73\end{array}$ & 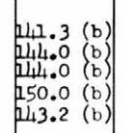 & $\begin{array}{l}\bar{z} \\
\bar{z}\end{array}$ & $\begin{array}{l}136 \\
\frac{1,11}{1141} \\
\frac{1142}{1143}\end{array}$ & $\begin{array}{r}96 \\
98 \\
98 \\
95 \\
100\end{array}$ & $\mid \begin{array}{l}0.233 \\
0.218 \\
0.221 \\
0.199 \\
0.194\end{array}$ & $\begin{array}{r}97 \\
100 \\
100 \\
99 \\
100\end{array}$ & $\begin{array}{l}- \\
1.9 \\
\overline{2.0} \\
0.14\end{array}$ & $\begin{array}{l}8.4 \\
7.9 \\
8.0 \\
7.2 \\
7.1\end{array}$ & $\begin{array}{r}15.9 \\
15.4 \\
19.1 \\
9.3 \\
15.2\end{array}$ & \begin{tabular}{r|}
23.0 \\
21.3 \\
30.5 \\
79.9 \\
19.6
\end{tabular} & \begin{tabular}{|l|}
1.4 \\
3.3 \\
2.2 \\
1.0 \\
1.8
\end{tabular} & \begin{tabular}{|l|}
2.7 \\
4.0 \\
3.2 \\
2.0 \\
2.3
\end{tabular} & $\begin{array}{l}1.92 \\
1.21 \\
1.45 \\
2.00 \\
1.28\end{array}$ & $\begin{array}{l}L-M \\
M \\
M \\
L \\
L-M\end{array}$ & $\begin{array}{l}\mathrm{T} \\
\mathrm{T} \\
\mathrm{T} \\
\mathrm{T} \\
\mathrm{SC}\end{array}$ \\
\hline $\begin{array}{l}\mathrm{AFG}-3 \\
\mathrm{AFG}-4 \\
\mathrm{MP}-2 \\
\mathrm{MP}-6 \\
\mathrm{AFC}-6\end{array}$ & $\begin{array}{l}\text { Afghantstan } \\
\text { Af hehansistan } \\
\text { Marble Point } \\
\text { Marble Point } \\
\text { Afghantstan }\end{array}$ & & $\begin{array}{r}1 \\
1 \\
2 \\
2 \\
1 \frac{1}{2} \\
\end{array}$ & $\begin{array}{l}57 \\
49 \\
45 \\
45 \\
55\end{array}$ & $\begin{array}{l}24 \\
18 \\
25 \\
25 \\
20 \\
\end{array}$ & $\begin{array}{l}10 \\
9.8 \\
11 \\
111 \\
11 \\
\end{array}$ & $\begin{array}{l}4.5 \\
6.5 \\
6.8 \\
6.8 \\
6.9 \\
\end{array}$ & $\begin{array}{l}3.3 \\
6.0 \\
6.0 \\
4.7 \\
\end{array}$ & $\begin{array}{l}2.4 \\
4.0 \\
4.0 \\
3.4\end{array}$ & \begin{tabular}{|l}
75 \\
71 \\
258 \\
258 \\
125 \\
\end{tabular} & $\begin{array}{l}1.5 \\
1.7 \\
0.7 \\
0.7 \\
3.1 \\
\end{array}$ & & & $\begin{array}{l}2.70 \\
2.72 \\
2.72 \\
2.72 \\
2.71 \\
\end{array}$ & $\begin{array}{c}146.1 \\
146.1 \\
139.3 \\
139.3 \\
139 \\
-\end{array}$ & $\begin{array}{l}z \\
\vdots \\
z\end{array}$ & $\begin{array}{l}1142 \\
1144 \\
135 \\
135 \\
144 \\
\end{array}$ & $\begin{array}{r}97 \\
98 \\
97 \\
97 \\
>95 \\
\end{array}$ & $\begin{array}{l}0.184 \\
0.175 \\
0.262 \\
0.260 \\
0.171 \\
\end{array}$ & $\begin{array}{r}96 \\
95 \\
100 \\
99 \\
99 \\
\end{array}$ & $\begin{array}{l}z \\
z \\
-\end{array}$ & $\begin{array}{l}6.8 \\
6.3 \\
9.6 \\
9.5 \\
9.2 \\
\end{array}$ & $\begin{array}{l}114.4 \\
12.4 \\
12.0 \\
14.2 \\
19.1 \\
\end{array}$ & $\begin{array}{l}18.0 \\
19.3 \\
7.0 \\
14.6 \\
24.7 \\
\end{array}$ & $\begin{array}{l}2.1 \\
4.2 \\
1.2 \\
1.2 \\
4.6 \\
\end{array}$ & \begin{tabular}{|l|}
2.4 \\
4.4 \\
2.3 \\
2.3 \\
7.6 \\
\end{tabular} & $\begin{array}{l}1.14 \\
1.04 \\
1.92 \\
1.92 \\
1.65 \\
\end{array}$ & \begin{tabular}{|l}
$\mathrm{M}$ \\
$\mathrm{H}$ \\
$\mathrm{L}-\mathrm{M}$ \\
$\mathrm{L} \mathrm{M}$ \\
$\mathrm{H}-\mathrm{M}$ \\
\end{tabular} & $\begin{array}{l}\mathrm{T} \\
\mathrm{T} \\
\mathrm{T} \\
\mathrm{T} \\
\mathrm{T} \\
\end{array}$ \\
\hline $\begin{array}{l}\text { AFG-2 } \\
\text { CBG-1 } \\
\text { BM-7 } \\
\text { BPR-4 }\end{array}$ & $\begin{array}{l}\text { Afghanistan } \\
\text { Cold Brook Pit } \\
\text { Bll Mountain Till } \\
\text { B.P.R. Alaska }\end{array}$ & GM & $\begin{array}{r}\frac{1}{2} \\
2 \frac{1}{2} \\
2 \\
1\end{array}$ & $\begin{array}{l}48 \\
55 \\
91 \\
58\end{array}$ & $\begin{array}{l}21 \\
28 \\
35 \\
38\end{array}$ & $\begin{array}{l}13 \\
15 \\
18 \\
27\end{array}$ & \begin{tabular}{|l|}
6.3 \\
6.3 \\
7.0 \\
10
\end{tabular} & $\begin{array}{l}4.4 \\
4.1 \\
5.0\end{array}$ & $\begin{array}{l}3.0 \\
2.0 \\
- \\
2.2\end{array}$ & $\begin{array}{l}193 \\
167 \\
250 \\
270\end{array}$ & $\begin{array}{l}3.6 \\
0.9 \\
0.3 \\
0.1\end{array}$ & & & $\begin{array}{l}2.72 \\
2.72 \\
2.81 \\
2.72\end{array}$ & $\begin{array}{l}144.3 \text { (b) } \\
144.7 \text { (b) }\end{array}$ & $\overline{-}$ & $\begin{array}{l}142 \\
139 \\
1145 \\
127\end{array}$ & $\begin{array}{l}98 \\
96 \\
96\end{array}$ & $\mid \begin{array}{l}0.191 \\
0.218 \\
0.210 \\
0.338\end{array}$ & $\begin{array}{r}96 \\
96 \\
100 \\
92\end{array}$ & $\begin{array}{l}\bar{z} \\
\overline{-}\end{array}$ & $\begin{array}{r}6.8 \\
7.6 \\
7.5 \\
11.4\end{array}$ & $\begin{array}{c}11.2 \\
20.9 \\
8.7 \\
26.9\end{array}$ & $\begin{array}{l}40.0 \\
10.7 \\
38.7\end{array}$ & $\begin{array}{l}2.2 \\
3.0 \\
0.7 \\
2.8\end{array}$ & \begin{tabular}{|l|}
3.0 \\
4.8 \\
2.2 \\
4.5
\end{tabular} & $\begin{array}{l}1.36 \\
1.60 \\
3.14 \\
1.60\end{array}$ & $\mid \begin{array}{l}M \\
M-H \\
V L-M \\
M-H\end{array}$ & $\begin{array}{l}\mathrm{T} \\
\mathrm{T} \\
\mathrm{T} \\
\mathrm{SC}\end{array}$ \\
\hline
\end{tabular}


Table AI (Cont'd). Summary of frost susceptibility tests on natural soils ${ }^{(1)}$ - open-system nominal load pressure 0.5 psi.

\begin{tabular}{|c|c|c|c|c|c|c|c|c|c|c|c|c|c|c|c|c|c|c|c|c|c|c|c|c|c|c|c|c|c|}
\hline \multirow{3}{*}{$\begin{array}{l}\text { Specimen } \\
\text { Number }\end{array}$} & \multirow{3}{*}{ Material Source } & \multicolumn{10}{|c|}{ SOIL GRADATION DATA (As Frozen) } & \multicolumn{5}{|c|}{ PHYSICAL PROPERTIES OF BASIC SOIL } & \multicolumn{5}{|c|}{ SPECIMEN DATA (As Molded) } & \multicolumn{7}{|c|}{ FREEZING TEST DATA } & \multirow{3}{*}{$\begin{array}{l}\text { Typ } \\
\text { of } \\
\text { Cyl } \\
\text { (12) }\end{array}$} \\
\hline & & \multirow{2}{*}{$\begin{array}{l}\text { Unified } \\
\text { Soli } \\
\text { clossifi- } \\
\text { cation } \\
\text { Symbol } \\
\text { (2) }\end{array}$} & \multirow{2}{*}{$\begin{array}{c}\substack{\text { Maxi- } \\
\text { mum } \\
\text { Size }} \\
\text { in. }\end{array}$} & \multicolumn{6}{|c|}{ Percent finer, mm } & \multicolumn{2}{|c|}{$\begin{array}{c}\text { Coetficients } \\
(3)\end{array}$} & \multicolumn{2}{|c|}{$=\begin{array}{l}\text { Atterbers } \\
\text { Limits (4) }\end{array}$} & \multirow{2}{*}{$\begin{array}{l}\text { Specific } \\
\text { Grovity }\end{array}$} & \multicolumn{2}{|c|}{$\begin{array}{c}\text { Compaction Dato } \\
(5)\end{array}$} & \multirow{2}{*}{$\begin{array}{c}\text { Dry } \\
\text { Unit } \\
\text { Woight } \\
\text { pct }\end{array}$} & \multirow{2}{*}{$\begin{array}{c}\text { Degree } \\
\text { of } \\
\text { Compoc- } \\
\text { tion } \\
\\
\%\end{array}$} & \multirow{2}{*}{\begin{tabular}{|} 
Void \\
Ratio \\
$\%$
\end{tabular}} & \multirow{2}{*}{$\begin{array}{l}\text { G, at } \\
\text { Stort } \\
\text { of } \\
\text { Tost } \\
(6) \\
\%\end{array}$} & \multirow{2}{*}{$\begin{array}{c}\text { Perme- } \\
\text { obilify } \\
k \\
(7) \\
\mathrm{cm} / \mathrm{sec} \\
\times 10^{-4}\end{array}$} & \multicolumn{2}{|c|}{$\begin{array}{l}\text { Avg. } \\
\text { Wottor } \\
\text { Content }\end{array}$} & \multirow{2}{*}{\begin{tabular}{|c|} 
\\
Totol \\
Heave \\
(8) \\
$\mathbf{6}$
\end{tabular}} & \multicolumn{2}{|c|}{$\begin{array}{l}\text { Rate of } \\
\text { Heove } \\
\text { mm/doy (9) }\end{array}$} & \multirow{2}{*}{\begin{tabular}{|l|} 
Heave \\
Rate \\
Vor \\
Index \\
(10)
\end{tabular}} & \multirow{2}{*}{$\begin{array}{l}\text { Erosi } \\
\text { Susc } \\
\text { Cusa } \\
\text { CliI) } \\
\text { (II) }\end{array}$} & \\
\hline & & & & 4.76 & 0.42 & 0.074 & 0.02 & 0.01 & 0.005 & $c_{u}$ & $c_{c}$ & LL & PI & & $\mid \begin{array}{c}\text { Moximum } \\
\text { Dry Unif } \\
\text { Weight } \\
\text { pcf }\end{array}$ & $\begin{array}{c}\text { Optimum } \\
\text { Moisture } \\
\text { Content } \\
\%\end{array}$ & & & & & & $\mid \begin{array}{c}\text { Before } \\
\text { Test } \\
\%\end{array}$ & $\begin{array}{c}\text { After } \\
\text { Tost } \\
\%\end{array}$ & & Avo. & Max. & & & \\
\hline & & & & & & & & & & & & CL & LAYEY & SANDY & RAVELS & & & & & & & & & & & & & & \\
\hline $\begin{array}{l}\text { WDG-1 } \\
\text { WDG-2 }\end{array}$ & $\begin{array}{l}\text { Washington, D.c. } \\
\text { Washington, D.c. }\end{array}$ & $G W-G C$ & $\frac{1 \frac{7}{2}}{1 \frac{2}{2}}$ & $\begin{array}{l}37 \\
37\end{array}$ & $\begin{array}{l}16 \\
16\end{array}$ & $\begin{array}{l}6.4 \\
6.4\end{array}$ & $\begin{array}{l}4.2 \\
4.2\end{array}$ & : & $=$ & $\begin{array}{l}57 \\
57\end{array}$ & $\begin{array}{l}2.5 \\
2.5\end{array}$ & $\begin{array}{r}26 \\
=86\end{array}$ & 9 & $\begin{array}{l}2.65 \\
2.65\end{array}$ & $\left|\begin{array}{ll}133.9 & \text { (d) } \\
133.9 & \text { (a) }\end{array}\right|$ & $\begin{array}{l}4.7 \\
4.7\end{array}$ & $\begin{array}{l}135 \\
136\end{array}$ & $\begin{array}{l}101 \\
101\end{array}$ & $\begin{array}{l}0.220 \\
0.218\end{array}$ & $\begin{array}{l}97 \\
96\end{array}$ & $=$ & $\begin{array}{l}8.0 \\
7.7\end{array}$ & $\begin{array}{l}12.7 \\
12.0\end{array}$ & $\begin{array}{l}15.6 \\
15.5\end{array}$ & $\begin{array}{l}2.1 \\
2.6\end{array}$ & $\begin{array}{l}3.0 \\
3.3\end{array}$ & $\begin{array}{l}1.42 \\
1.26\end{array}$ & $\begin{array}{l}M \\
M\end{array}$ & $\begin{array}{l}\mathrm{T} \\
\mathrm{T}\end{array}$ \\
\hline $\begin{array}{l}\text { PI-2 } \\
\text { PI-4 }\end{array}$ & $\begin{array}{l}\text { Presque Isle } \\
\text { Presque Isle }\end{array}$ & GP-GC & $\begin{array}{l}3 / 4 \\
3 / 4\end{array}$ & $\begin{array}{l}37 \\
33 \\
\end{array}$ & $\begin{array}{l}14 \\
15 \\
\end{array}$ & $\frac{11}{12}$ & $\begin{array}{l}6.6 \\
8.7 \\
\end{array}$ & $\begin{array}{l}5.0 \\
6.9 \\
\end{array}$ & 3.2 & $\begin{array}{l}145 \\
315\end{array}$ & $\begin{array}{l}16 \\
32\end{array}$ & $\begin{array}{r}24.9 \\
22.3 \\
\end{array}$ & $\begin{array}{l}8.7 \\
8.1 \\
\end{array}$ & $\begin{array}{l}2.72 \\
2.75 \\
\end{array}$ & 136.8 (b) & - & $\begin{array}{l}134 \\
131\end{array}$ & $\begin{array}{l}98 \\
96 \\
\end{array}$ & $\begin{array}{l}0.265 \\
0.250 \\
\end{array}$ & $\begin{array}{l}97 \\
98 \\
\end{array}$ & - & $\begin{array}{l}9.7 \\
8.8 \\
\end{array}$ & $\begin{array}{l}22.3 \\
16: 2\end{array}$ & $\begin{array}{l}42.5 \\
19.9 \\
\end{array}$ & $\begin{array}{l}2.9 \\
1.5\end{array}$ & $\begin{array}{l}3.7 \\
2.0\end{array}$ & $\begin{array}{l}1.28 \\
1.33 \\
\end{array}$ & $\underset{L}{M}$ & $\begin{array}{l}\mathrm{SC} \\
\mathrm{SC}\end{array}$ \\
\hline CL-1 & Clinton County & $G M-G C$ & $1 \frac{1}{2}$ & 54 & 30 & 20 & 15 & 9.0 & 5.0 & 485 & 1.9 & $248 \mathrm{CL}^{2}$ & 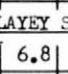 & $\begin{array}{l}\text { SILIY GR } \\
2.74\end{array}$ & RAVEIS & 9.0 & 129 & 99 & 0.320 & 100 & 0.1 & 11.7 & 30.3 & 65.6 & 4.6 & 5.7 & 1.24 & H & sc \\
\hline $\begin{array}{l}\text { GF-1 } \\
\text { LST-18 } \\
\text { LST-19 } \\
\text { LST-20 } \\
\text { LST-21 } \\
\text { LST-34 }\end{array}$ & $\begin{array}{l}\text { Great Falls } \\
\text { Lorine } \\
\text { Loring } \\
\text { Loring } \\
\text { Loring } \\
\text { Loring }\end{array}$ & GC & $\begin{array}{l}1 \frac{2}{2} \\
3 / 4 \\
3 / 4 \\
3 / 4 \\
3 / 4 \\
3 / 4\end{array}$ & $\begin{array}{l}48 \\
68 \\
68 \\
68 \\
68 \\
68\end{array}$ & $\begin{array}{l}36 \\
52 \\
52 \\
52 \\
52 \\
52\end{array}$ & $\begin{array}{l}22 \\
41 \\
41 \\
41 \\
41 \\
41\end{array}$ & $\begin{array}{l}17 \\
30 \\
30 \\
30 \\
30 \\
30\end{array}$ & $\begin{array}{l}15 \\
25 \\
25 \\
25 \\
25 \\
25\end{array}$ & $\begin{array}{l}12 \\
18 \\
18 \\
18 \\
18 \\
18\end{array}$ & $\begin{array}{l}4000 \\
945 \\
945 \\
945 \\
9445 \\
945\end{array}$ & $\begin{array}{l}1.2 \\
0.1 \\
0.1 \\
0.1 \\
0.1 \\
0.1\end{array}$ & $\left|\begin{array}{l}42.6 \\
22.1 \\
22.1 \\
22.1 \\
22.1 \\
22.1\end{array}\right|$ & $\begin{array}{r}\text { CLAY: } \\
24.6 \\
7.8 \\
7.8 \\
7.8 \\
7.8 \\
7.8\end{array}$ & $\begin{array}{l}\text { YEY GRAVE } \\
\begin{array}{|l}2.66 \\
2.73 \\
2.73 \\
2.73 \\
2.73 \\
2.73\end{array}\end{array}$ & 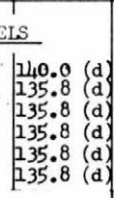 & $\begin{array}{l}5.6 \\
7.5 \\
7.5 \\
7.5 \\
7.5 \\
7.5\end{array}$ & $\begin{array}{l}133 \\
129 \\
132 \\
136 \\
134 \\
132\end{array}$ & $\begin{array}{r}95 \\
95 \\
97 \\
100 \\
99 \\
97\end{array}$ & $\mid \begin{array}{l}0.252 \\
0.320 \\
0.290 \\
0.250 \\
0.270 \\
0.290\end{array}$ & $\begin{array}{l}100 \\
100 \\
100 \\
100 \\
100 \\
95\end{array}$ & $\begin{array}{c}.00003 \\
= \\
= \\
= \\
-\end{array}$ & $\begin{array}{r}9.5 \\
11.5 \\
10.3 \\
9.0 \\
9.7 \\
10.0\end{array}$ & $\begin{array}{l}21.0 \\
34.8 \\
19.0 \\
17.6 \\
24.3 \\
32.0\end{array}$ & $\begin{array}{l}28.0 \\
84.4 \\
30.2 \\
28.8 \\
42.5 \\
81.9\end{array}$ & $\begin{array}{l}2.4 \\
4.0 \\
2.3 \\
1.5 \\
2.6 \\
4.9\end{array}$ & $\begin{array}{r}5.0 \\
6.8 \\
3.7 \\
2.7 \\
4.0 \\
13.2\end{array}$ & $\begin{array}{l}2.08 \\
1.70 \\
1.50 \\
1.80 \\
1.54 \\
2.69\end{array}$ & \begin{tabular}{|l|}
$M-H I$ \\
$H$ \\
$M$ \\
LMM \\
$M$ \\
$H-V H$
\end{tabular} & \begin{tabular}{|l} 
SC \\
SC \\
$S C$ \\
SC \\
SC \\
SC
\end{tabular} \\
\hline $\begin{array}{l}\text { PAF-3 } \\
\text { PAF-4 } \\
\text { PAF-7 } \\
\text { PC-1 } \\
\text { PC-3 } \\
\text { PAF-5 } \\
\text { PAF-6 }\end{array}$ & $\begin{array}{l}\text { Plattsburg } \\
\text { Plattsburg } \\
\text { Plattsburg } \\
\text { Fairchild } \\
\text { Fairchild. } \\
\text { Plattsburg } \\
\text { Plattsburg }\end{array}$ & $\mathrm{SP}$ & $\begin{array}{l}1 \frac{1}{2} \\
1 \frac{1}{2} \\
1 \\
2 \\
2 \\
1 \frac{1}{2} \\
1 \frac{\frac{1}{2}}{2}\end{array}$ & $\begin{array}{l}59 \\
59 \\
72 \\
85 \\
70 \\
72 \\
72\end{array}$ & \begin{tabular}{|c|}
20 \\
20 \\
7.0 \\
8.6 \\
6.9 \\
36 \\
36 \\
\end{tabular} & \begin{tabular}{|l|}
2.1 \\
2.1 \\
3.0 \\
3.6 \\
3.4 \\
4.5 \\
4.5 \\
\end{tabular} & $\begin{array}{l}1.0 \\
1.0 \\
1.3 \\
1.3 \\
1.4 \\
1.8 \\
1.8 \\
\end{array}$ & \begin{tabular}{|l|}
0.8 \\
0.8 \\
0.9 \\
1.2 \\
1.3 \\
1.4 \\
1.4 \\
\end{tabular} & $\begin{array}{c}0.5 \\
0.5 \\
0.5 \\
- \\
1.0 \\
1.0 \\
\end{array}$ & \begin{tabular}{c|c}
24 & \\
24 & \\
5.3 & \\
3.4 & \\
4.7 & \\
5.1 & \\
5.1 &
\end{tabular} & $\begin{array}{l}0.3 \\
0.3 \\
2.0 \\
0.2 \\
1.3 \\
0.7 \\
0.7\end{array}$ & & & $\begin{array}{l}2.67 \\
2.67 \\
3.20 \\
2.74 \\
2.74 \\
2.67 \\
2.67\end{array}$ & $\begin{array}{l}132.8(\mathrm{~b}) \\
132.8(\mathrm{~b}) \\
139.1(\mathrm{~b}) \\
119.2(\mathrm{~b}) \\
132.1(\mathrm{~b}) \\
125.2(\mathrm{~b}) \\
125.2(\mathrm{~b})\end{array}$ & $\begin{array}{l}- \\
\vdots \\
\vdots \\
-\end{array}$ & $\begin{array}{l}130 \\
130 \\
139 \\
116 \\
125 \\
124 \\
125 \\
\end{array}$ & $\begin{array}{r}98 \\
98 \\
200 \\
98 \\
95 \\
99 \\
100 \\
\end{array}$ & $\begin{array}{l}0.281 \\
0.283 \\
0.440 \\
0.469 \\
0.368 \\
0.338 \\
0.329 \\
\end{array}$ & $\begin{array}{r}100 \\
100 \\
86 \\
100 \\
100 \\
95 \\
90 \\
\end{array}$ & $\begin{array}{l}- \\
\vdots \\
- \\
-\end{array}$ & $\begin{array}{l}10.5 \\
10.6 \\
11.7 \\
17.0 \\
13.4 \\
12.0 \\
12.3\end{array}$ & \begin{tabular}{|l|}
11.2 \\
12.8 \\
11.7 \\
19.0 \\
15.4 \\
12.0 \\
13.9
\end{tabular} & \begin{tabular}{|r|}
6.0 \\
9.6 \\
7.5 \\
10.4 \\
10.8 \\
5.3 \\
9.8
\end{tabular} & \begin{tabular}{|l|}
0.6 \\
0.3 \\
0.3 \\
0.8 \\
0.7 \\
0.6 \\
0.7
\end{tabular} & $\begin{array}{l}0.7 \\
0.4 \\
0.4 \\
1.6 \\
1.1 \\
0.8 \\
0.9\end{array}$ & $\begin{array}{l}1.16 \\
1.33 \\
1.33 \\
2.00 \\
1.57 \\
1.33 \\
1.28\end{array}$ & $\left\{\begin{array}{l}\text { VL } \\
N \\
N \\
\text { VL-L } \\
\text { VL-L. } \\
\text { VL } \\
\text { VL }\end{array}\right.$ & \begin{tabular}{|l}
$\mathrm{SC}$ \\
$\mathrm{SC}$ \\
$\mathrm{SC}$ \\
$\mathrm{SC}$ \\
$\mathrm{T}$ \\
$\mathrm{T}$ \\
$\mathrm{SC}$ \\
$\mathrm{SC}$
\end{tabular} \\
\hline
\end{tabular}


Table AI (Cont'd).

\begin{tabular}{|c|c|c|c|c|c|c|c|c|c|c|c|c|c|c|c|c|c|c|c|c|c|c|c|c|c|c|c|c|c|}
\hline \multirow{3}{*}{$\begin{array}{l}\text { Specimen } \\
\text { Number }\end{array}$} & \multirow{3}{*}{ Moterial Source } & \multicolumn{10}{|c|}{ SOIL GRADATION DATA (As Frozen) } & \multicolumn{5}{|c|}{ PHYSIEAL PROPERTIES OF BASIC SOIL } & \multicolumn{5}{|c|}{ SPECIMEN DATA (AS Molded) } & \multicolumn{7}{|c|}{ FREEZING TEST DATA } & \multirow{3}{*}{$\begin{array}{l}\text { Type } \\
\text { of } \\
\text { Cyl. } \\
\text { (12) }\end{array}$} \\
\hline & & \multirow{2}{*}{$\begin{array}{l}\text { Unified } \\
\text { Soil } \\
\text { Clossifi- } \\
\text { cotion } \\
\text { Symbol } \\
\text { (2) }\end{array}$} & \multirow{2}{*}{$\begin{array}{l}\text { Maxi- } \\
\text { mum } \\
\text { Size } \\
\text { in. }\end{array}$} & \multicolumn{6}{|c|}{ Percent finer, mm } & \multicolumn{2}{|c|}{$\begin{array}{c}\text { Coefficients } \\
(3)\end{array}$} & \multicolumn{2}{|c|}{$\begin{array}{l}\text { Atterberg } \\
\text { Limits (4) }\end{array}$} & \multirow{2}{*}{$\begin{array}{l}\text { Specific } \\
\text { Grovity } \\
-\end{array}$} & \multicolumn{2}{|c|}{$\begin{array}{c}\text { Compoction Dato } \\
(5)\end{array}$} & \multirow{2}{*}{$\begin{array}{c}\text { Dry } \\
\text { Unit } \\
\text { Weight } \\
\text { pcf }\end{array}$} & \multirow{2}{*}{$\begin{array}{c}\text { Degree } \\
\text { of } \\
\text { compoc- } \\
\text { tion } \\
\%\end{array}$} & \multirow{2}{*}{$\begin{array}{c}\text { Void } \\
\text { Ratio } \\
\%\end{array}$} & \multirow{2}{*}{$\begin{array}{l}\text { G, at } \\
\text { stort } \\
\text { of } \\
\text { Tost } \\
(6) \\
\%\end{array}$} & \multirow{2}{*}{$\begin{array}{c}\text { Perme- } \\
\text { obility } \\
k \\
(7) \\
\mathrm{cm} / \mathrm{sec} \\
\times 10^{-4}\end{array}$} & \multicolumn{2}{|c|}{$\begin{array}{l}\text { Avg. } \\
\text { Woffor } \\
\text { Content }\end{array}$} & \multirow{2}{*}{$\begin{array}{c}\text { Total } \\
\text { Heave } \\
(8) \\
\%\end{array}$} & \multicolumn{2}{|c|}{\begin{tabular}{|l|}
$\begin{array}{l}\text { Rote of of } \\
\text { Heove } \\
\text { mm/doy (9) }\end{array}$
\end{tabular}} & \multirow{2}{*}{$\begin{array}{l}\text { Heave } \\
\text { Heave } \\
\text { Rate } \\
\text { Vor. } \\
\text { Index } \\
(10)\end{array}$} & \multirow{2}{*}{$\begin{array}{l}\text { Erost } \\
\text { Fusc. } \\
\text { Cusc } \\
\text { (II) } \\
\text { (II) }\end{array}$} & \\
\hline & & & & 4.76 & 0.42 & 0.074 & 0.02 & 0.01 & 0.005 & $c_{u}$ & $\mathrm{c}_{\mathrm{c}}$ & LL & PI & & $\left|\begin{array}{c}\text { Moximum } \\
\text { Ory Unit } \\
\text { weight } \\
\text { pct }\end{array}\right|$ & $\begin{array}{c}\text { Optimum } \\
\text { moisture } \\
\text { Content } \\
\%\end{array}$ & & & & & & \begin{tabular}{|c|} 
Before \\
Test \\
$\%$
\end{tabular} & $\mid \begin{array}{c}\text { After } \\
\text { Tost } \\
\%\end{array}$ & & Avg. & Mox. & & & \\
\hline $\begin{array}{l}\text { KA }-5 \\
\text { BPR-2 } \\
\text { SPK-1 } \\
\text { MIN-1 } \\
\text { SA -2 }\end{array}$ & $\begin{array}{l}\text { Keflavik } \\
\text { B. P. R., Alaska } \\
\text { Spdkne } \\
\text { Minnesota } \\
\text { Stewart }\end{array}$ & SW-SM & $\begin{array}{l}3 / 4 \\
1 / 4 \\
1 \frac{1}{2} \\
2 \\
2\end{array}$ & $\begin{array}{l}57 \\
68 \\
68 \\
69 \\
58\end{array}$ & $\begin{array}{l}16 \\
12 \\
11 \\
20 \\
26\end{array}$ & $\begin{array}{l}5.0 \\
5.6 \\
7.0 \\
9.6 \\
9.1\end{array}$ & $\begin{array}{l}1.4 \\
2.9 \\
3.5 \\
3.8 \\
4.0\end{array}$ & $\mid$\begin{tabular}{c|}
- \\
2.3 \\
2.3 \\
- \\
2.9
\end{tabular} & $\begin{array}{l}- \\
1.8 \\
1.2 \\
- \\
1.8\end{array}$ & $\mid$\begin{tabular}{l|}
27 \\
10 \\
$6 \cdot 7$ \\
28 \\
31
\end{tabular} & $\begin{array}{l}1.1 \\
1.0 \\
1.4 \\
1.8 \\
1.1\end{array}$ & SII & $\mid$ & \begin{tabular}{|l} 
PA VELLY S \\
2.81 \\
2.75 \\
2.80 \\
2.73 \\
2.70
\end{tabular} & 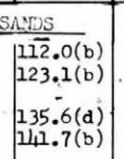 & $\begin{array}{c}- \\
\vdots \\
6.5 \\
-\end{array}$ & $\begin{array}{l}111 \\
117 \\
128 \\
135 \\
139\end{array}$ & $\begin{array}{r}99 \\
95 \\
975 \\
295 \\
106 \\
98\end{array}$ & $\begin{array}{l}0.532 \\
0.467 \\
0.365 \\
0.258 \\
0.214\end{array}$ & $\begin{array}{r}100 \\
93 \\
100 \\
97 \\
100\end{array}$ & $\begin{array}{c}- \\
- \\
- \\
3.5\end{array}$ & $\begin{array}{l}19.5 \\
15.8 \\
13.0 \\
9.4 \\
8.5\end{array}$ & \begin{tabular}{|l|}
19.3 \\
21.0 \\
15.8 \\
22.0 \\
18.2
\end{tabular} & \begin{tabular}{|r|}
2.0 \\
15.7 \\
13.6 \\
37.0 \\
27.9
\end{tabular} & \begin{tabular}{|l|}
0.3 \\
1.2 \\
1.1 \\
2.8 \\
4.4
\end{tabular} & $\begin{array}{l}0.5 \\
1.8 \\
2.0 \\
4.3 \\
5.0\end{array}$ & $\begin{array}{l}1.65 \\
1.50 \\
1.82 \\
1.54 \\
1.35\end{array}$ & \begin{tabular}{|l} 
R \\
$L$ \\
$L$ \\
$Y-H$ \\
$Y H$
\end{tabular} & $\begin{array}{l}\mathrm{sc} \\
\mathrm{sc} \\
\mathrm{sc} \\
m \\
m\end{array}$ \\
\hline $\begin{array}{l}\text { SA }-6 \\
\text { MIT }-4 \\
\text { HDG }-6 \\
\text { HDC }-12 \\
\text { HDG }-13\end{array}$ & $\begin{array}{l}\text { Stewart } \\
\text { M. I. T. } \\
\text { Hutchinson's P1t } \\
\text { Hutchinson's pit } \\
\text { Hutchinson's Pit }\end{array}$ & & $\begin{array}{l}2 \\
1 \frac{1}{2} \\
1 \\
1 \\
1\end{array}$ & \begin{tabular}{|l|}
68 \\
70 \\
57 \\
57 \\
57 \\
57
\end{tabular} & $\begin{array}{l}26 \\
29 \\
20 \\
20 \\
20\end{array}$ & $\begin{array}{l}9.1 \\
9.7 \\
8.7 \\
8.7 \\
8.7\end{array}$ & $\begin{array}{l}4.0 \\
4.4 \\
5.0 \\
5.0 \\
5.0\end{array}$ & $\begin{array}{l}2.9 \\
3.2 \\
3.5 \\
3.5 \\
3.5\end{array}$ & $\begin{array}{l}1.8 \\
2.5 \\
2.0 \\
2.0 \\
2.0\end{array}$ & $\begin{array}{l}31 \\
24 \\
43 \\
43 \\
43\end{array}$ & $\begin{array}{l}1.1 \\
1.2 \\
1.1 \\
1.1 \\
1.1\end{array}$ & $19 \cdot 3$ & 4.3 & $\begin{array}{l}2.70 \\
2.70 \\
2.75 \\
2.75 \\
2.75\end{array}$ & $\begin{array}{l}1111.7(\mathrm{~b}) \\
137.9(\mathrm{~b}) \\
113.3(\mathrm{c}) \\
113.3(\mathrm{c}) \\
113.3(\mathrm{c})\end{array}$ & $\begin{array}{c}- \\
5.3 \\
5.3 \\
5.3\end{array}$ & $\begin{array}{l}138 \\
131 \\
144 \\
141 \\
138\end{array}$ & $\begin{array}{r}98 \\
95 \\
101 \\
98 \\
96\end{array}$ & $\begin{array}{l}0.224 \\
0.285 \\
0.179 \\
0.221 \\
0.242\end{array}$ & $\begin{array}{r}100 \\
97 \\
99 \\
87 \\
99\end{array}$ & $\begin{array}{l}4.0 \\
- \\
0.02 \\
0.13 \\
0.29\end{array}$ & $\begin{array}{r}8.5 \\
10.2 \\
6.7 \\
7.0 \\
8.7\end{array}$ & \begin{tabular}{|l|}
20.4 \\
20.7 \\
24.7 \\
37.0 \\
23.3
\end{tabular} & \begin{tabular}{|l|}
32.2 \\
21.9 \\
49.7 \\
81.3 \\
43.8
\end{tabular} & \begin{tabular}{|l|}
2.7 \\
1.2 \\
6.1 \\
4.8 \\
3.5
\end{tabular} & $\begin{array}{l}4.3 \\
2.0 \\
7.7 \\
5.8 \\
4.8\end{array}$ & $\begin{array}{l}1.3 \\
1.66 \\
1.26 \\
1.20 \\
1.37\end{array}$ & $\begin{array}{l}\because-H \\
\mathrm{~L} \\
\vdots \\
H \\
\mathrm{H} \\
\mathrm{Y}-\mathrm{H}\end{array}$ & ì \\
\hline $\begin{array}{l}\text { LSG }-38 \\
\text { RC-1 } \\
\text { AFG }-7 \\
\text { GR }-4 \\
\text { FC }-2\end{array}$ & $\begin{array}{l}\text { Loring } \\
\text { Rapid C1ty } \\
\text { Afghanistan } \\
\text { Greenland } \\
\text { Fairchild }\end{array}$ & SP-SM & $\begin{array}{l}\frac{1}{2} \\
1 \frac{1}{2} \\
2 \\
3 / 4 \\
2\end{array}$ & \begin{tabular}{|l|}
62 \\
57 \\
58 \\
58 \\
60 \\
84 \\
\end{tabular} & $\begin{array}{l}13 \\
30 \\
23 \\
39 \\
2.1\end{array}$ & $\begin{array}{l}7.2 \\
22 \\
8.2 \\
9.7 \\
5.3\end{array}$ & $\begin{array}{l}5.7 \\
8.7 \\
3.7 \\
1.8 \\
1.9\end{array}$ & $\begin{array}{l}5.0 \\
7.1 \\
2.3 \\
0.8 \\
1.7\end{array}$ & $\begin{array}{c}4.0 \\
5.8 \\
1.8 \\
- \\
-\end{array}$ & \begin{tabular}{c|}
15 \\
283 \\
48 \\
62 \\
4.0
\end{tabular} & $\begin{array}{l}1.3 \\
1.1 \\
1.2 \\
0.2 \\
1.6\end{array}$ & 19.0 & 2.0 & $\begin{array}{l}2.71 \\
2.75 \\
2.71 \\
2.73 \\
2.75\end{array}$ & $\mid \begin{array}{c}139.1(b) \\
- \\
146.7(b) \\
138.0(d) \\
123.5(b)\end{array}$ & $\begin{array}{c}- \\
- \\
5.3 \\
-\end{array}$ & $\begin{array}{l}135 \\
137 \\
147 \\
137 \\
121\end{array}$ & $\begin{array}{r}97 \\
>95 \\
>100 \\
199 \\
98\end{array}$ & $\begin{array}{l}0.256 \\
0.253 \\
0.150 \\
0.246 \\
0.421\end{array}$ & $\begin{array}{r}98 \\
98 \\
100 \\
100 \\
100\end{array}$ & $\begin{array}{l}- \\
0.048 \\
-.48 \\
-\end{array}$ & $\begin{array}{r}9.2 \\
8.9 \\
5.4 \\
9.0 \\
15.3\end{array}$ & \begin{tabular}{|l|}
24.7 \\
13.9 \\
16.4 \\
16.9 \\
17.9
\end{tabular} & \begin{tabular}{|l|}
37.4 \\
16.4 \\
31.3 \\
20.4 \\
10.8
\end{tabular} & \begin{tabular}{|l|}
3.3 \\
1.7 \\
3.7 \\
1.3 \\
0.9
\end{tabular} & \begin{tabular}{l|}
4.7 \\
2.5 \\
5.3 \\
1.8 \\
1.5
\end{tabular} & $\begin{array}{l}1.42 \\
1.47 \\
1.43 \\
1.38 \\
1.66\end{array}$ & $\mid$\begin{tabular}{l|}
$M-H$ \\
$L-M$ \\
$Y-H$ \\
$L$ \\
VL-L \\
\end{tabular} & $\begin{array}{l}\mathrm{SC} \\
\mathrm{SC} \\
- \\
- \\
?\end{array}$ \\
\hline $\begin{array}{l}\text { MIN-4 } \\
\text { VF-6 } \\
\text { DU-1 } \\
\text { DU-2 } \\
\text { DU-3 }\end{array}$ & $\begin{array}{l}\text { Minnesota } \\
\text { Vo: } k \text { Field } \\
\text { Indiana } \\
\text { Indiana } \\
\text { Indiana }\end{array}$ & & $\begin{array}{l}- \\
- \\
- \\
-\end{array}$ & $\begin{array}{l}100 \\
100 \\
100 \\
100 \\
100\end{array}$ & $\begin{array}{l}71 \\
86 \\
100 \\
100 \\
100\end{array}$ & $\begin{array}{l}8.8 \\
5.0 \\
5.3 \\
6.3 \\
6.3\end{array}$ & $\begin{array}{l}2.2 \\
2.6 \\
2.6 \\
2.6 \\
2.6\end{array}$ & $\begin{array}{l}1.3 \\
2.4 \\
2.2 \\
2.2 \\
2.2\end{array}$ & $\begin{array}{l}- \\
1.8 \\
1.7 \\
1.7 \\
1.7\end{array}$ & \begin{tabular}{l|l}
4.3 \\
2.0 \\
1.9 \\
1.9 \\
1.9
\end{tabular} & $\begin{array}{l}1.5 \\
0.9 \\
1.0 \\
1.0 \\
1.0\end{array}$ & & & $\begin{array}{l}2.70 \\
2.65 \\
2.65 \\
2.05 \\
2.65\end{array}$ & $\begin{array}{l}114.4(\mathrm{~b}) \\
115.6(\mathrm{~b}) \\
107.1(\mathrm{~b}) \\
107.1(\mathrm{~b}) \\
107.1(\mathrm{~b})\end{array}$ & $\begin{array}{l}- \\
- \\
- \\
-\end{array}$ & $\begin{array}{l}114 \\
115 \\
109 \\
105 \\
109\end{array}$ & $\begin{array}{r}100 \\
100 \\
102 \\
98 \\
102\end{array}$ & $\begin{array}{l}0.473 \\
0.450 \\
0.516 \\
0.575 \\
0.514\end{array}$ & $\begin{array}{l}100 \\
100 \\
100 \\
100 \\
100\end{array}$ & $\begin{array}{c}- \\
- \\
26.0 \\
38.0 \\
26.0\end{array}$ & $\begin{array}{l}16: 8 \\
12.3 \\
19.3 \\
21.8 \\
19.4\end{array}$ & $\begin{array}{l}16.0 \\
16.3 \\
16.7 \\
19.8 \\
20.5\end{array}$ & $\begin{array}{l}2.4 \\
2.8 \\
0.7 \\
1.3 \\
2.3\end{array}$ & \begin{tabular}{l|}
0.2 \\
0.1 \\
0.1 \\
0.1 \\
0.4
\end{tabular} & $\begin{array}{l}0.5 \\
0.5 \\
0.5 \\
0.5 \\
0.5\end{array}$ & $\begin{array}{l}2.50 \\
5.00 \\
.00 \\
.00 \\
1.25\end{array}$ & 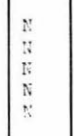 & $\begin{array}{l}\vdots \\
\text { sc } \\
\text { sc } \\
\text { sc }\end{array}$ \\
\hline $\begin{array}{l}\text { MI-1 } \\
\text { DFB-1 } \\
\text { SLF-1 } \\
\text { SLF-2 } \\
\text { SCA-1 }\end{array}$ & $\begin{array}{l}\text { Minot } \\
\text { Dow Field } \\
\text { Selfridge } \\
\text { Selfridge } \\
\text { Schenectady }\end{array}$ & & $\begin{array}{l}1 \frac{1}{2} \\
3 / 4 \\
1 / 2 \\
1 \frac{2}{2} \\
3 / 4\end{array}$ & $\begin{array}{l}73 \\
66 \\
74 \\
77 \\
99\end{array}$ & $\begin{array}{l}11 \\
18 \\
25 \\
27 \\
84\end{array}$ & $\begin{array}{l}5.2 \\
6.0 \\
5.9 \\
7.1 \\
10\end{array}$ & $\begin{array}{l}2.7 \\
2.8 \\
3.2 \\
3.3 \\
3.3\end{array}$ & $\begin{array}{l}2.2 \\
1.7 \\
2.7 \\
3.0 \\
3.0\end{array}$ & $\begin{array}{l}1.6 \\
1.0 \\
1.8 \\
2.6 \\
2.0\end{array}$ & \begin{tabular}{|c|}
8.1 \\
15 \\
15 \\
13 \\
3.4
\end{tabular} & $\begin{array}{l}0.9 \\
0.9 \\
0.6 \\
0.7 \\
1.8\end{array}$ & & & $\begin{array}{l}2.73 \\
2.72 \\
2.70 \\
2.70 \\
2.58\end{array}$ & $\begin{array}{l}130.5(\mathrm{~b}) \\
137.6(\mathrm{~b}) \\
126.8(\mathrm{~b}) \\
126.8(\mathrm{~b}) \\
113.0(\mathrm{~b})\end{array}$ & $\begin{array}{l}\vdots \\
\vdots \\
-\end{array}$ & $\begin{array}{l}129 \\
133 \\
127 \\
127 \\
113\end{array}$ & $\begin{array}{r}99 \\
97 \\
100 \\
100 \\
100\end{array}$ & $\begin{array}{l}0.316 \\
0.278 \\
0.329 \\
0.329 \\
0.484\end{array}$ & $\begin{array}{r}100 \\
100 \\
100 \\
100 \\
98\end{array}$ & $\begin{array}{c}- \\
0.2 \\
= \\
-\end{array}$ & $\begin{array}{l}11.5 \\
10.7 \\
12.2 \\
12.2 \\
17.7\end{array}$ & \begin{tabular}{l|}
14.3 \\
21.8 \\
19.9 \\
20.7 \\
25.5
\end{tabular} & $\begin{array}{r}8.8 \\
27.6 \\
18.3 \\
18.0 \\
16.5\end{array}$ & $\begin{array}{l}0.5 \\
1.8 \\
1.0 \\
1.2 \\
1.1\end{array}$ & $\begin{array}{l}1.0 \\
3.3 \\
1.7 \\
2.2 \\
2.2\end{array}$ & $\begin{array}{l}2.00 \\
2.83 \\
1.70 \\
1.83 \\
2.00\end{array}$ & $\begin{array}{l}\text { VL } \\
\text { L-M } \\
\text { L } \\
\text { L-M } \\
\text { L-M }\end{array}$ & $\begin{array}{l}\mathrm{SC} \\
\mathrm{sC}\end{array}$ \\
\hline $\begin{array}{l}\text { SCA-2 } \\
\text { KIS-6 } \\
\text { KIS-2 } \\
\text { KIS-3 } \\
\text { HDG -1 }\end{array}$ & $\begin{array}{l}\text { Schenectady } \\
\text { Kinross } \\
\text { Kinross } \\
\text { Kinross } \\
\text { Hutchinson's P1t }\end{array}$ & & $\begin{array}{l}3 / 4 \\
1 \frac{1}{2} \\
- \\
- \\
2\end{array}$ & $\begin{array}{r}97 \\
98 \\
100 \\
100 \\
56\end{array}$ & $\begin{array}{l}84 \\
80 \\
82 \\
82 \\
17\end{array}$ & $\begin{array}{l}10 \\
8.8 \\
9.0 \\
9.0 \\
6.0\end{array}$ & $\begin{array}{l}3.3 \\
3.3 \\
3.4 \\
3.4 \\
3.4 \\
3.5\end{array}$ & $\begin{array}{l}3.0 \\
2.0 \\
2.0 \\
2.0 \\
2.4\end{array}$ & $\begin{array}{l}2.0 \\
0.9 \\
0.9 \\
0.9 \\
-\end{array}$ & $\mid$\begin{tabular}{r|}
3.4 \\
2.8 \\
2.8 \\
2.8 \\
28
\end{tabular} & $\begin{array}{l}1.8 \\
1.4 \\
1.4 \\
1.4 \\
0.7\end{array}$ & & & $\begin{array}{l}2.68 \\
2.62 \\
2.62 \\
2.63 \\
2.74\end{array}$ & $\begin{array}{l}113.0(\mathrm{~b}) \\
109.0(\mathrm{~b}) \\
109.0(\mathrm{~b}) \\
109.0(\mathrm{~b}) \\
141.0(\mathrm{~b})\end{array}$ & $\begin{array}{l}\vdots \\
\vdots\end{array}$ & $\begin{array}{l}112 \\
108 \\
106 \\
105 \\
140\end{array}$ & $\begin{array}{l}99 \\
99 \\
98 \\
97 \\
99\end{array}$ & $\begin{array}{l}0.487 \\
0.518 \\
0.542 \\
0.552 \\
0.222\end{array}$ & $\begin{array}{l}100 \\
100 \\
100 \\
100 \\
100\end{array}$ & $\begin{array}{c}- \\
- \\
- \\
0.1\end{array}$ & $\begin{array}{l}18.2 \\
19.8 \\
20.6 \\
20.4 \\
18.1\end{array}$ & \begin{tabular}{|l|}
26.2 \\
21.5 \\
20.9 \\
21.1 \\
18.2
\end{tabular} & $\begin{array}{r}17.5 \\
6.2 \\
3.3 \\
4.0 \\
28.1 \\
\end{array}$ & $\begin{array}{l}1.0 \\
0.7 \\
0.4 \\
0.4 \\
3.7\end{array}$ & $\begin{array}{l}2.0 \\
1.2 \\
0.7 \\
0.8 \\
5.5\end{array}$ & $\begin{array}{l}2.00 \\
1.71 \\
1.75 \\
2.00 \\
1.48\end{array}$ & $\left|\begin{array}{l}\mathrm{L} \\
\mathrm{VL}-\mathrm{L} \\
\mathrm{N}-\mathrm{VL} \\
\mathrm{N}-\mathrm{VL} \\
\mathrm{H}\end{array}\right|$ & $\mid \begin{array}{l}\text { sc: } \\
+ \\
-\end{array}$ \\
\hline $\begin{array}{l}K-2 A \\
K-2 B \\
\text { LIN-2 } \\
\text { SPK-3 } \\
\text { SPK-4 }\end{array}$ & $\begin{array}{l}\text { Korea } \\
\text { Korea } \\
\text { Lincoln } \\
\text { Spokane } \\
\text { Spokane }\end{array}$ & & $\begin{array}{l}2 \frac{1}{4} \\
2 \frac{1}{2} \\
1 \\
3,4 \\
3 / 4\end{array}$ & $\begin{array}{l}58 \\
56 \\
56 \\
79 \\
79\end{array}$ & $\begin{array}{l}28 \\
28 \\
22 \\
13 \\
13\end{array}$ & \begin{tabular}{|l|}
9.4 \\
9.4 \\
6.5 \\
8.1 \\
8.1 \\
\end{tabular} & $\begin{array}{l}3.5 \\
3.5 \\
3.9 \\
4.1 \\
4.1\end{array}$ & \begin{tabular}{l|}
2.1 \\
2.1 \\
2.7 \\
2.7 \\
2.7
\end{tabular} & $\begin{array}{l}1.4 \\
1.4 \\
2.0 \\
1.5 \\
1.5\end{array}$ & \begin{tabular}{|c|}
211 \\
111 \\
17 \\
6.4 \\
6.4
\end{tabular} & $\begin{array}{l}0.3 \\
0.3 \\
0.9 \\
3.2 \\
3.2\end{array}$ & & & $\begin{array}{l}2.61 \\
2.61 \\
2.65 \\
2.80 \\
2.80\end{array}$ & $\begin{array}{c}127.0(\mathrm{~b}) \\
127.0(\mathrm{~b}) \\
134.0(\mathrm{~d}) \\
-\end{array}$ & $\begin{array}{l}- \\
\vdots \\
-\end{array}$ & $\begin{array}{l}128 \\
124 \\
134 \\
128 \\
128\end{array}$ & $\begin{array}{r}100 \\
98 \\
100 \\
<95 \\
<95\end{array}$ & $\begin{array}{l}0.268 \\
0.310 \\
0.238 \\
0.361 \\
0.351\end{array}$ & $\begin{array}{r}96 \\
99 \\
100 \\
100 \\
90\end{array}$ & $\begin{array}{l}- \\
\vdots \\
-\end{array}$ & $\begin{array}{r}9.6 \\
11.9 \\
9.0 \\
12.6 \\
11.3\end{array}$ & $\begin{array}{l}13.0 \\
17.3 \\
13.7 \\
17.7 \\
18.5\end{array}$ & \begin{tabular}{|l|}
45.2 \\
20.8 \\
14.0 \\
13.5 \\
16.3
\end{tabular} & $\begin{array}{l}2.2 \\
3.8 \\
0.8 \\
1.1 \\
1.4\end{array}$ & $\begin{array}{l}3.5 \\
5.0 \\
1.4 \\
1.4 \\
2.3\end{array}$ & $\begin{array}{l}1.59 \\
1.32 \\
1.75 \\
1.27 \\
1.64\end{array}$ & $\begin{array}{l}\text { M } \\
\text { M-H } \\
\text { VL-L } \\
\text { L } \\
\text { L-M }\end{array}$ & $\begin{array}{l}3 \\
\mathrm{SC} \\
\mathrm{SC} \\
\mathrm{SC}\end{array}$ \\
\hline $\begin{array}{l}\text { KIS - } \\
\text { KIS -4 } \\
\text { KIS-5 } \\
\text { PBJ-3 } \\
\text { PBJ-4 }\end{array}$ & $\begin{array}{l}\text { Kinross } \\
\text { Kinross } \\
\text { Kinross } \\
\text { Proj. Blue Jay } \\
\text { Proj. Blue Jay }\end{array}$ & & $\begin{array}{l}3 / 4 \\
3 / 4 \\
1 \frac{1}{2} \\
3 / 4 \\
3 / 4\end{array}$ & $\begin{array}{l}92 \\
92 \\
92 \\
71 \\
71\end{array}$ & $\begin{array}{l}67 \\
67 \\
67 \\
46 \\
46\end{array}$ & $\begin{array}{c}9.0 \\
9.0 \\
9.0 \\
10 \\
10\end{array}$ & $\begin{array}{l}4.5 \\
4.5 \\
4.5 \\
4.5 \\
4.5\end{array}$ & $\begin{array}{l}2.9 \\
2.9 \\
2.9 \\
4.0 \\
4.0\end{array}$ & \begin{tabular}{|l|}
1.8 \\
1.8 \\
1.8 \\
1.8 \\
1.8
\end{tabular} & \begin{tabular}{|l|}
4.2 \\
4.2 \\
4.2 \\
20 \\
20
\end{tabular} & $\begin{array}{l}1.2 \\
1.2 \\
1.2 \\
0.3 \\
0.3\end{array}$ & & & $\begin{array}{l}2.65 \\
2.65 \\
2.65 \\
2.70 \\
2.70\end{array}$ & $\begin{array}{l}120.4 \text { (b) } \\
220.4 \text { (b) } \\
220.4 \text { (b) } \\
1142.6 \text { (b) } \\
1142.6 \text { (b) }\end{array}$ & $\begin{array}{l}- \\
\vdots \\
-\end{array}$ & $\begin{array}{l}115 \\
119 \\
120 \\
138 \\
137\end{array}$ & $\begin{array}{r}95 \\
98 \\
100 \\
97 \\
96\end{array}$ & $\begin{array}{l}0.438 \\
0.396 \\
0.367 \\
0.215 \\
0.230\end{array}$ & $\begin{array}{r}100 \\
100 \\
99 \\
100 \\
100\end{array}$ & $\begin{array}{l}\vdots \\
\vdots\end{array}$ & \begin{tabular}{|r|}
16.5 \\
14.9 \\
13.9 \\
8.0 \\
8.5
\end{tabular} & \begin{tabular}{|l|}
19.3 \\
31.1 \\
32.9 \\
25.8 \\
37.8
\end{tabular} & $\begin{array}{r}8.2 \\
36.5 \\
44.4 \\
29.0 \\
69.4\end{array}$ & \begin{tabular}{|l|}
0.8 \\
2.7 \\
5.4 \\
3.1 \\
3.2
\end{tabular} & $\begin{array}{l}1.7 \\
3.7 \\
7.8 \\
4.5 \\
5.8\end{array}$ & $\begin{array}{l}2.12 \\
1.37 \\
1.44 \\
1.45 \\
1.81\end{array}$ & \begin{tabular}{|l|} 
VL-L \\
$M$ \\
$\mathrm{H}$ \\
$\mathrm{MH}$ \\
$\mathrm{MHE}$ \\
\end{tabular} & $\begin{array}{l}\mathrm{T} \\
\mathrm{T} \\
\mathrm{T} \\
\mathrm{SC} \\
\mathrm{SC}\end{array}$ \\
\hline $\begin{array}{l}\text { T-3 } \\
\text { LIN-3 } \\
\text { LIN-1 } \\
\text { LIN-4 } \\
\text { CDB-3 }\end{array}$ & $\begin{array}{l}\text { Tobyhanna } \\
\text { Lincoln } \\
\text { Lincoln } \\
\text { Lincoln } \\
\text { Cape Dyer }\end{array}$ & & $\begin{array}{l}1_{2}^{\frac{1}{2}} \\
1 \\
1 \\
1 \\
2\end{array}$ & $\begin{array}{l}59 \\
80 \\
63 \\
71 \\
61\end{array}$ & $\begin{array}{l}39 \\
24 \\
30 \\
27 \\
29\end{array}$ & $\begin{array}{l}8.5 \\
6.5 \\
7.0 \\
7.8 \\
9.7\end{array}$ & $\begin{array}{l}4.5 \\
4.9 \\
5.0 \\
5.0 \\
5.1\end{array}$ & $\begin{array}{l}2.5 \\
3.8 \\
3.0 \\
4.0 \\
4.2\end{array}$ & \begin{tabular}{|l|}
1.6 \\
3.0 \\
2.0 \\
3.2 \\
3.1 \\
3.1 \\
1
\end{tabular} & $\begin{array}{l}6.0 \\
15 \\
28 \\
16 \\
52\end{array}$ & $\begin{array}{l}0.2 \\
0.8 \\
0.4 \\
0.6 \\
0.7\end{array}$ & & & $\begin{array}{l}2.72 \\
2.65 \\
2.65 \\
2.65 \\
2.68\end{array}$ & $\begin{array}{l}140.4 \text { (b) } \\
133.1 \text { (d) } \\
133.1 \text { (d) } \\
133.1 \text { (d) } \\
134.8 \text { (b) }\end{array}$ & $\begin{array}{l}- \\
- \\
-\end{array}$ & $\begin{array}{l}134 \\
135 \\
137 \\
132 \\
130\end{array}$ & $\begin{array}{r}96 \\
101 \\
103 \\
99 \\
97\end{array}$ & $\begin{array}{l}0.280 \\
0.228 \\
0.212 \\
0.250 \\
0.289\end{array}$ & $\begin{array}{r}100 \\
98 \\
100 \\
98 \\
94\end{array}$ & $\begin{array}{l}: \\
\vdots \\
-\end{array}$ & $\begin{array}{r}9.9 \\
8.6 \\
8.0 \\
9.3 \\
10.1\end{array}$ & $\begin{array}{l}20.2 \\
13.5 \\
12.7 \\
15.5 \\
24.1\end{array}$ & $\begin{array}{l}21.8 \\
14.9 \\
15.8 \\
19.6 \\
37.3\end{array}$ & \begin{tabular}{|l|}
1.5 \\
1.0 \\
1.0 \\
1.2 \\
2.1
\end{tabular} & \begin{tabular}{|l|}
2.3 \\
1.4 \\
1.4 \\
1.7 \\
3.3
\end{tabular} & $\begin{array}{l}1.53 \\
1.40 \\
1.40 \\
1.42 \\
1.57\end{array}$ & $\begin{array}{l}L-M \\
L \\
L \\
L \\
M\end{array}$ & $\begin{array}{l}\text { SC } \\
\text { SC } \\
\text { SC } \\
\text { SC }\end{array}$ \\
\hline $\begin{array}{l}\text { AFG }-1 \\
\text { WVS-3 } \\
\text { VF-7 } \\
\text { LSG }-15 \\
\text { LSG }-39\end{array}$ & $\begin{array}{l}\text { Afghanistan } \\
\text { West V1rginia } \\
\text { Volk Field } \\
\text { loring } \\
\text { Loring }\end{array}$ & & $\begin{array}{l}1 \\
1 \frac{1}{2} \\
2 \\
1\end{array}$ & $\begin{array}{l}71 \\
57 \\
94 \\
65 \\
88\end{array}$ & $\begin{array}{l}32 \\
33 \\
83 \\
14 \\
17\end{array}$ & $\begin{array}{l}11.9 \\
10 \\
10 \\
8.5 \\
10 \\
\end{array}$ & $\begin{array}{l}5.5 \\
5.6 \\
5.6 \\
7.2 \\
8.2 \\
\end{array}$ & $\begin{array}{l}3.9 \\
4.5 \\
5.0 \\
6.2 \\
7.2 \\
\end{array}$ & \begin{tabular}{|c|c|}
2.8 \\
- \\
3.6 \\
4.6 \\
5.8
\end{tabular} & $\mid$\begin{tabular}{c|}
81 \\
3.0 \\
260 \\
29 \\
27 \\
\end{tabular} & $\begin{array}{l}0.3 \\
0.3 \\
1.5 \\
34 \\
14.9 \\
\end{array}$ & 24 & 6 & $\begin{array}{l}2.73 \\
2.70 \\
2.62 \\
2.71 \\
2.72\end{array}$ & $\begin{array}{l}-143.2 \text { (b) } \\
129.1 \text { (b) } \\
221.6 \text { (b) } \\
139.1 \text { (b) } \\
130.1 \text { (b) }\end{array}$ & $\begin{array}{l}- \\
\vdots \\
-\end{array}$ & $\begin{array}{l}141 \\
125 \\
120 \\
135 \\
135\end{array}$ & $\begin{array}{l}- \\
97 \\
97 \\
97 \\
97 \\
\end{array}$ & $\begin{array}{l}0.205 \\
0.349 \\
0.364 \\
0.254 \\
0.259 \\
\end{array}$ & $\begin{array}{r}96 \\
87 \\
100 \\
93 \\
99 \\
\end{array}$ & $\begin{array}{c}- \\
2.3 \\
-\end{array}$ & $\begin{array}{r}7.2 \\
11.3 \\
13.9 \\
8.5 \\
8.5 \\
\end{array}$ & \begin{tabular}{|l|}
19.8 \\
23.4 \\
31.2 \\
14.6 \\
31.6
\end{tabular} & $\begin{array}{l}35.5 \\
27.6 \\
39.2 \\
17.4 \\
59.8 \\
\end{array}$ & \begin{tabular}{|l|}
3.9 \\
1.8 \\
2.3 \\
2.0 \\
2.0 \\
\end{tabular} & \begin{tabular}{|l|}
7.1 \\
3.3 \\
3.2 \\
3.8 \\
4.2 \\
\end{tabular} & $\begin{array}{l}1.82 \\
1.83 \\
1.39 \\
1.90 \\
2.10 \\
\end{array}$ & $\mid$\begin{tabular}{l}
$M H$ \\
$L-M$ \\
$M$ \\
\hdashline \\
$M I I$ \\
\end{tabular} & $\begin{array}{l}T \\
T \\
T \\
\text { SC } \\
\text { EC } \\
\end{array}$ \\
\hline
\end{tabular}


Table AI (Cont'd). Summary of frost susceptibility tests on natural soils ${ }^{(1)}$ - open-system nominal load pressure 0.5 psi.

\begin{tabular}{|c|c|c|c|c|c|c|c|c|c|c|c|c|c|c|c|c|c|c|c|c|c|c|c|c|c|c|c|c|c|}
\hline \multirow{3}{*}{$\mid \begin{array}{c}\text { Specimon } \\
\text { Number }\end{array}$} & \multirow{3}{*}{ Material Source } & \multicolumn{10}{|c|}{ SOIL GRADATION DATA (AS Frozen) } & \multicolumn{5}{|c|}{ PHYSICAL PROPERTIES OF BASIC SOIL } & \multicolumn{5}{|c|}{ SPECIMEN DATA (AS MOIded) } & \multicolumn{7}{|c|}{ FREEZING TEST DATA } & \multirow{3}{*}{$\begin{array}{l}\text { st } \\
\text { s. } \\
\text { sis }\end{array}$} \\
\hline & & \multirow{2}{*}{$\begin{array}{l}\text { Unifitiod } \\
\text { choii } \\
\text { clossifi- } \\
\text { cotion } \\
\text { Symbol } \\
\text { (2) }\end{array}$} & \multirow[b]{2}{*}{\begin{tabular}{|c|} 
Maxi- \\
mum \\
Size \\
in.
\end{tabular}} & \multicolumn{6}{|c|}{ Percent finer, $\mathrm{mm}$} & \multicolumn{2}{|c|}{$\begin{array}{c}\text { Coefficient } \\
\text { (3) }\end{array}$} & \multicolumn{2}{|c|}{$\begin{array}{l}\text { Arterbero } \\
\text { Limits (4) }\end{array}$} & \multirow{2}{*}{$\begin{array}{l}\text { Specific } \\
\text { Grovity }\end{array}$} & \multicolumn{2}{|c|}{\begin{tabular}{|c|} 
Compoction Dato \\
$(5)$
\end{tabular}} & \multirow{2}{*}{$\begin{array}{c}\text { Dry } \\
\text { unnit } \\
\text { Woight } \\
\text { pct }\end{array}$} & \multirow{2}{*}{$\begin{array}{c}\text { Dogree } \\
\text { of } \\
\text { compoc- } \\
\text { tion } \\
\boldsymbol{x} \\
\end{array}$} & \multirow{2}{*}{\begin{tabular}{|c|} 
Void \\
Ratio \\
$\%$ \\
\end{tabular}} & \multirow{2}{*}{$\begin{array}{l}\text { G, ot } \\
\text { storf } \\
\text { of } \\
\text { Tost } \\
(6) \\
\%\end{array}$} & \multirow{2}{*}{\begin{tabular}{|c|} 
Porme- \\
obilify \\
$k$ \\
$(7)$ \\
$\mathrm{cm} / \mathrm{sec}$ \\
$\times 10^{-4}$
\end{tabular}} & \multicolumn{2}{|c|}{$\begin{array}{c}\text { Avg. } \\
\text { worter } \\
\text { contont } \\
\end{array}$} & \multirow{2}{*}{$\begin{array}{c}\text { Totol } \\
\text { Heove } \\
\text { (8) } \\
\% \\
\%\end{array}$} & & & & & \\
\hline & & & & 4.76 & 0.42 & 0.074 & 0.02 & 0.01 & 0.005 & 5 . $c_{u}$ & $c_{c}$ & LL & PI & & $\mid \begin{array}{c}\text { Moximum } \\
\text { Orive nuf } \\
\text { weight } \\
\text { pct }\end{array}$ & $\left\{\begin{array}{c}\text { Ootimum } \\
\text { Moisture } \\
\text { content } \\
\%\end{array}\right.$ & & & & & & \begin{tabular}{|l} 
Before \\
Test \\
$\%$
\end{tabular} & $\begin{array}{l}\text { Afror } \\
\text { Tost } \\
\%\end{array}$ & & Avg. & Max. & Index & & \\
\hline & & SM & & & & & & & & & & & & ILTY SAITR. & & & & & & & & & & & & & & & \\
\hline 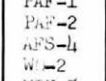 & $\begin{array}{l}\text { Patitso } \\
\text { Patesta } \\
\text { Alasta } \\
\text { lestove }\end{array}$ & $S M$ & $\bar{z}$ & $\begin{array}{l}\mid \begin{array}{l}100 \\
100 \\
100 \\
100\end{array} \\
100\end{array}$ & $\begin{array}{l}95 \\
95 \\
100 \\
86\end{array}$ & $\begin{array}{l}28 \\
28 \\
33 \\
20\end{array}$ & $\begin{array}{l}1.5 \\
2.5 \\
2.5 \\
2.5\end{array}$ & $\mid \begin{array}{l}1 \\
1.2 \\
-2 \\
-\end{array}$ & $\begin{array}{l}0.9 \\
0.9 \\
-\end{array}$ & $\begin{array}{l}2.5 \\
2.5 \\
1.6 \\
1.1\end{array}$ & $\begin{array}{l}0.9 \\
0.9 \\
1.0\end{array}$ & & & $\begin{array}{l}2.68 \\
2.68 \\
2.79 \\
2.69\end{array}$ & 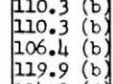 & 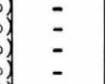 & $\begin{array}{l}107 \\
109 \\
108 \\
115\end{array}$ & $\begin{array}{l}97 \\
99 \\
101 \\
96\end{array}$ & 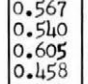 & \begin{tabular}{|l}
85 \\
96 \\
200 \\
99
\end{tabular} & $\overline{-}$ & $\begin{array}{l}118.6 \\
19.2 \\
21.7 \\
16.5\end{array}$ & $\begin{array}{l}18.7 \\
19.2 \\
24.6 \\
17.6\end{array}$ & $\begin{array}{l}4.4 \\
4.4 \\
9.4 \\
4.3\end{array}$ & $\mid \begin{array}{l}0.2 \\
0.1 \\
0.7 \\
0.2\end{array}$ & $\mid \begin{array}{l}0.5 \\
0.2 \\
1.5 \\
0.5 \\
0.3\end{array}$ & $\begin{array}{l}2.50 \\
2.00 \\
2.114 \\
2.14 \\
1.50\end{array}$ & $\mid \begin{array}{l}N \\
N \\
V_{L}-L\end{array}$ & $\begin{array}{l}s c \\
s c \\
s c \\
s c\end{array}$ \\
\hline$n+110-5$ & Minne & & - & 100 & 95 & 20 & 3.8 & 2.2 & - & 3.7 & & & & 2.68 & 114.2 (d) & - & 114 & 100 & 0.434 & gو & & 16.0 & $\begin{aligned} 42.8 \\
2.8\end{aligned}-10$ & .8 & 2.3 & 9.3 & 4.04 & ${ }_{M-V H}^{N}$ & $T_{T}^{S}$ \\
\hline 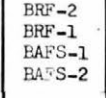 & $\begin{array}{l}\text { Eradiey } \\
\text { Bradey } \\
\text { Bethel } \\
\text { Dethel }\end{array}$ & & $\begin{array}{l}3 / 4 \\
3 / 4 \\
-\end{array}$ & $\begin{array}{l}79 \\
67 \\
100 \\
100\end{array}$ & $\begin{array}{l}27 \\
31 \\
100 \\
100\end{array}$ & $\begin{array}{l}1,14 \\
14 \\
21 \\
21\end{array}$ & $\begin{array}{l}4.2 .2 \\
4.4 \\
4.5 \\
4.5\end{array}$ & $\begin{array}{l}2.6 \\
2.6 \\
2.5 \\
2.5\end{array}$ & $\begin{array}{l}= \\
1.0 \\
1.0\end{array}$ & $\begin{array}{l}47 \\
62 \\
3.0 \\
3.0 \\
\end{array}$ & : $: 9$ & & & $\begin{array}{l}2.76 \\
2.76 \\
2.68 \\
2.68\end{array}$ & $\begin{array}{l}133.6 \text { (b } \\
133.1 \\
130.1 \text { (b) } \\
106.4 \text { (d) } \\
106.4 \text { (d) }\end{array}$ & $\bar{z}$ & $\begin{array}{l}\text { l33- } \\
\text { 143 } \\
106 \\
105\end{array}$ & $\mid \begin{array}{l}100 \\
100 \\
100 \\
99\end{array}$ & {$\left[\begin{array}{l}0.300 \\
0.202 \\
0.578 \\
0.593\end{array}\right]$} & $\begin{array}{l}100 \\
100 \\
96 \\
88\end{array}$ & $\vdots$ & $\begin{array}{l}10.9 \\
7.3 \\
20.7 \\
19.4\end{array}$ & $\begin{array}{l}20.6 \\
21.9 \\
21.6 \\
21.6\end{array}$ & $\mid \begin{array}{l}20.0 \\
21.9 \\
21.9 \\
21.6 \\
21.6\end{array}$ & $\begin{array}{l}1.2 \\
2.4 \\
0.5 \\
0.6\end{array}$ & $\begin{array}{l}1.6 \\
3.3 \\
1.0 \\
1.0\end{array}$ & $\begin{array}{l}1.33 \\
1.33 \\
2.00 \\
1.66\end{array}$ & $\mid \begin{array}{l}\mathrm{L} \\
\mathrm{M} \\
\mathrm{V} \\
\mathrm{VL}\end{array}$ & $\begin{array}{l}T \\
T \\
T \\
T\end{array}$ \\
\hline $\begin{array}{l}\text { WO-3 } \\
\mathrm{C}-1 \\
\mathrm{CR}-1\end{array}$ & & & $\begin{array}{l}3 / 4 \\
3 / 4\end{array}$ & $\begin{array}{r}100 \\
66 \\
66\end{array}$ & \begin{tabular}{|l}
86 \\
45 \\
45
\end{tabular} & $\begin{array}{l}26 \\
17 \\
17\end{array}$ & \begin{tabular}{|l|}
5.1 \\
5.2 \\
5.2
\end{tabular} & 3.7 & $\begin{array}{l}-.4 \\
2.4\end{array}$ & \begin{tabular}{|l}
27 \\
47 \\
47
\end{tabular} & & & & $\begin{array}{l}2.69 \\
2.73\end{array}$ & & & $\begin{array}{l}114 \\
135\end{array}$ & $\begin{array}{l}100 \\
98\end{array}$ & $\begin{array}{l}0.467 \\
0.258\end{array}$ & $\begin{array}{l}100 \\
100\end{array}$ & - & $\begin{array}{r}17.7 \\
9.5\end{array}$ & $\begin{array}{l}23.9 \\
22.6\end{array}$ & $\begin{array}{l}14.2 \\
35.3\end{array}$ & \begin{tabular}{l|l}
2 & 0.7 \\
3 & 2.2
\end{tabular} & $\begin{array}{l}1.0 \\
2.7\end{array}$ & $\begin{array}{l}1.42 \\
1.22\end{array}$ & ${ }_{M}^{\mathrm{VL}}$ & $\begin{array}{l}\mathrm{SC} \\
\mathrm{T}\end{array}$ \\
\hline 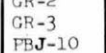 & & & $\begin{array}{l}3 / 4 \\
3 / 4 \\
3 / 4\end{array}$ & \begin{tabular}{|l|}
60 \\
66 \\
82
\end{tabular} & $\begin{array}{l}45 \\
45 \\
53\end{array}$ & $\begin{array}{l}17 \\
17 \\
21\end{array}$ & $\left|\begin{array}{l}5.2 \\
5.2 \\
6.0\end{array}\right|$ & $\begin{array}{l}3.7 \\
3.7 \\
5.2\end{array}$ & $\mid \begin{array}{l}2.4 \\
2.4 \\
2.8\end{array}$ & $\begin{array}{l}47 \\
47 \\
25\end{array}$ & & & & $\begin{array}{l}2.73 \\
2.73 \\
2.71\end{array}$ & $\begin{array}{l}137.9(\mathrm{~b}) \\
137.9(\mathrm{~b}) \\
136\end{array}$ & $\therefore$ & $\begin{array}{l}137 \\
136\end{array}$ & $\begin{array}{l}99 \\
99\end{array}$ & $\begin{array}{l}0.244 \\
0.252\end{array} \mid$ & $\begin{array}{r}97 \\
100\end{array}$ & : & $\begin{array}{l}8.6 \\
9.2\end{array}$ & $\begin{array}{l}31.6 \\
22.9\end{array}$ & \begin{tabular}{|l|}
60.2 \\
38.4
\end{tabular} & \begin{tabular}{l|l} 
& 3.8 \\
& 2.0
\end{tabular} & $\begin{array}{l}5.5 \\
2.9\end{array}$ & $\begin{array}{l}1.44 \\
1.45\end{array}$ & $\begin{array}{c}M-H \\
M\end{array}$ & $T_{T}^{2}$ \\
\hline FAG -5 & A & & $\begin{array}{l}3 / 4 \\
\frac{1}{2}\end{array}$ & \begin{tabular}{|l}
02 \\
57 \\
88
\end{tabular} & $\begin{array}{l}33 \\
23 \\
43\end{array}$ & $\begin{array}{l}21 \\
12 \\
18\end{array}$ & $\mid$\begin{tabular}{l|}
0.0 .2 \\
6.5
\end{tabular} & $\left|\begin{array}{l}3.4 \\
3.9 \\
4.0\end{array}\right|$ & $\begin{array}{l}2.0 \\
2.7 \\
2.5\end{array}$ & $\mid \begin{array}{l}23 \\
1111 \\
28.6\end{array}$ & & & & $\begin{array}{l}2.71 \\
2.69 \\
2.58\end{array}$ & $\begin{array}{l}136.0 \mathrm{cc}) \\
141.6(\mathrm{~b}) \\
120.0(\mathrm{~b})\end{array}$ & 7.0 & $\begin{array}{l}129 \\
144 \\
120\end{array}$ & $\begin{array}{l}95 \\
99 \\
99\end{array}$ & $\begin{array}{l}0.312 \\
0.155 \\
0.38\end{array}$ & 88 & & $\begin{array}{r}10.1 \\
6.0 \\
12.5\end{array}$ & $\begin{array}{l}28.5 \\
13.9\end{array}$ & $\begin{array}{l}36.9 \\
24.7 \\
\end{array}$ & $\begin{array}{l}9 \\
2.2\end{array}$ & 3.8 & $\begin{array}{l}1.31 \\
1: 68\end{array}$ & $M$ & $\mathrm{Sc}_{\mathrm{T}}$ \\
\hline $\begin{array}{l}0-4 \\
0.6 \\
-6\end{array}$ & & & & $\begin{array}{l}100 \\
100 \\
84\end{array}$ & $\begin{array}{l}85 \\
85\end{array}$ & $\begin{array}{l}27 \\
27\end{array}$ & $\begin{array}{r}7.0 \\
7.0\end{array}$ & & & $\begin{array}{l}6.9 \\
6.9\end{array}$ & 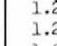 & & & i. & & $=$ & $\begin{array}{l}117 \\
111\end{array}$ & $\begin{array}{l}108 \\
95\end{array}$ & $\begin{array}{l}0.340 \\
0.450 \\
0.521\end{array}$ & $\begin{array}{l}100 \\
100\end{array}$ & $=$ & $\mid \begin{array}{ll}12.5 \\
10: 5 \\
i ; . .2\end{array}$ & $\begin{array}{l}26.2 \\
26: 5 \\
22.4\end{array}$ & $\mid \begin{array}{l}39: 5 \\
19: 0 \\
: 0.8\end{array}$ & $\begin{array}{l}5 \\
5 \\
3\end{array}$ & $\begin{array}{l}8: 8 \\
1.8 \\
1.3\end{array}$ & $\begin{array}{l}1: 45 \\
1: 50 \\
2.16\end{array}$ & $\underset{\text { VL-1 }}{\mathrm{H}}$ & $\begin{array}{l}\mathrm{SC} \\
\mathrm{SL}\end{array}$ \\
\hline $1 T-2$ & $\begin{array}{l}\text { M. } \\
\text { M. } \\
\text { I. }\end{array}$ & & $\frac{2}{2}$ & $\begin{array}{l}84 \\
76\end{array}$ & $\begin{array}{l}47 \\
49\end{array}$ & $\begin{array}{lll}13 \\
17\end{array}$ & $\begin{array}{l}7.5 \\
7.8\end{array}$ & \begin{tabular}{|c|c|}
3.3 \\
4.5
\end{tabular} & $\begin{array}{l}3.6 \\
3.0\end{array}$ & $\begin{array}{l}17 \\
28\end{array}$ & & & & $\begin{array}{l}2.70 \\
2.70\end{array}$ & $\mid \begin{array}{l}123.0(\mathrm{~d}) \\
122.1(\mathrm{~d})\end{array}$ & $\begin{array}{l}13.2 \\
14.2\end{array}$ & $\begin{array}{l}123 . \\
122 .\end{array}$ & 100 & 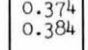 & $\begin{array}{l}96 \\
100\end{array}$ & & $\begin{array}{l}13.2 \\
14.2\end{array}$ & $\begin{array}{l}21.9 \\
25.3\end{array}$ & \begin{tabular}{|l|}
22.4 \\
28.3
\end{tabular} & \begin{tabular}{l|l}
4 & 2.3 \\
1 & 1.9
\end{tabular} & $\begin{array}{l}3.2 \\
2.7\end{array}$ & $\begin{array}{l}1.39 \\
1.42\end{array}$ & & $\frac{T}{T}$ \\
\hline $\begin{array}{l}\text { PAFB-7 } \\
\text { MNN-8 } \\
\text { WN-1 }\end{array}$ & & & $\frac{3 / 4}{2}$ & $\begin{array}{r}98 \\
100 \\
58\end{array}$ & \begin{tabular}{|l}
94 \\
97 \\
27
\end{tabular} & $\begin{array}{l}29 \\
48 \\
14\end{array}$ & \begin{tabular}{|l|}
8.2 \\
8.8 \\
8.9
\end{tabular} & \begin{tabular}{|l|}
5.4 \\
4.5 \\
7.5
\end{tabular} & $\begin{array}{l}3.7 \\
6.0\end{array}$ & $\begin{array}{l}4.0 \\
4.44 \\
250\end{array}$ & $\begin{array}{l}1.8 \\
0.8 \\
0.8\end{array}$ & 8 & 3.0 & $\begin{array}{l}2.73 \\
2.72 \\
2.70\end{array}$ & $\begin{array}{l}111.2(\mathrm{~d}) \\
126.0 \mathrm{~b} \\
129.4 \text { (b) }\end{array}$ & $=$ & $\begin{array}{l}109 \\
120 \\
128\end{array}$ & $\begin{array}{r}98 \\
95 \\
-99\end{array}$ & $\begin{array}{l}0.560 \\
0.419 \\
0.312\end{array}$ & $\begin{array}{r}96 \\
99 \\
100\end{array}$ & $\overline{-}$ & $\begin{array}{l}199.8 \\
15.3 \\
11.5\end{array}$ & \begin{tabular}{|l}
26.2 \\
22.0 \\
22.4
\end{tabular} & $\begin{array}{r}13.5 \\
18.3 \\
8.5\end{array}$ & \begin{tabular}{l|l}
5 \\
3 \\
3 \\
1.4 \\
0.9
\end{tabular} & $\begin{array}{l}1.5 \\
3.3 \\
1.2\end{array}$ & $\begin{array}{l}1.86 \\
2.36 \\
1.33\end{array}$ & $\mid \begin{array}{l}\mathrm{V} L-M \\
\mathrm{~L}-\mathrm{M} \\
\mathrm{VL}-\mathrm{M}\end{array}$ & $\begin{array}{l}\mathrm{l} \\
\mathrm{sc} \\
\mathrm{l} \\
\mathrm{sc}\end{array}$ \\
\hline & $\begin{array}{l}\text { Volk } \\
\text { Vonst }\end{array}$ & & $3 / 4$ & $\begin{array}{r}100 \\
78\end{array}$ & $\begin{array}{l}88 \\
53\end{array}$ & $\begin{array}{l}13 \\
23\end{array}$ & $\begin{array}{l}11 \\
11\end{array}$ & $\begin{array}{l}9.5 \\
7.5\end{array}$ & $\begin{array}{l}7.7 \\
4.5\end{array}$ & $\begin{array}{l}20 \\
20 \\
38\end{array}$ & $\begin{array}{l}7.5 \\
1.3 \\
=3\end{array}$ & & & $\begin{array}{l}2.72 \\
2.70\end{array}$ & $\begin{array}{l}119.5(\mathrm{~b}) \\
136.0(\mathrm{~d})\end{array}$ & $=$ & $\begin{array}{l}114 \\
131\end{array}$ & $\begin{array}{l}95 \\
96\end{array}$ & $\begin{array}{l}0.375 \\
0.290\end{array}$ & $\begin{array}{l}100 \\
98\end{array}$ & 0.097 & $\begin{array}{l}16.7 \\
10.5\end{array}$ & $\begin{array}{l}33.5 \\
30.1 \\
30.1\end{array}$ & $\begin{array}{l}37.2 \\
45.0\end{array}$ & $\begin{array}{l}2.2 \\
3.3\end{array}$ & $\begin{array}{l}1.06 \\
3.0 \\
4: 0\end{array}$ & $\begin{array}{l}1.36 \\
1.21 \\
1.21\end{array}$ & $\begin{array}{l}M \\
M\end{array}$ & $\begin{array}{l}\text { se } \\
\text { sc } \\
\text { sc }\end{array}$ \\
\hline $\begin{array}{l}\mathrm{H}-2 \\
\mathrm{HWC}-2\end{array}$ & $\begin{array}{l}\text { Mansfield Hollow } \\
\text { Fairch11d } \\
\text { Thule }\end{array}$ & & $\begin{array}{l}3 / 4 \\
3 / 4 \\
3 / 4\end{array}$ & $\begin{array}{l}78 \\
71 \\
73\end{array}$ & $\begin{array}{l}53 \\
34 \\
47\end{array}$ & $\begin{array}{l}23 \\
23 \\
20\end{array}$ & $\begin{array}{l}11 \\
11 \\
12\end{array}$ & $\begin{array}{l}7.5 \\
6.3 \\
9.0\end{array}$ & $\begin{array}{l}4.5 \\
4.0 \\
6.9\end{array}$ & $\begin{array}{l}38 \\
95 \\
71\end{array}$ & & & $\left|\begin{array}{l}2.0 \\
2.2\end{array}\right|$ & $\begin{array}{l}2.70 \\
2.79 \\
2.88\end{array}$ & & $=$ & $\begin{array}{l}131 \\
136 \\
145\end{array}$ & $\begin{array}{l}96 \\
95 \\
96\end{array}$ & $\begin{array}{l}0.291 \\
0.280 \\
0.043\end{array}$ & $\begin{array}{r}88 \\
100 \\
100\end{array}$ & 0.098 & $\begin{array}{r}9.5 \\
10.0 \\
8.4\end{array}$ & $\begin{array}{l}24.2 \\
22.2 \\
30.2\end{array}$ & $\mid \begin{array}{l}35.3 \\
27.1 \\
58.1\end{array}$ & $\begin{array}{ll}4.6 \\
2.8 \\
2.6\end{array}$ & $\begin{array}{l}6.5 \\
4.8 \\
.3\end{array}$ & $\begin{array}{l}1.41 \\
1.71 \\
.04\end{array}$ & M-H & $\begin{array}{l}3 \mathrm{sc} \\
\text { sc } \\
\text { sc }\end{array}$ \\
\hline & & & $\begin{array}{l}3 / 4 \\
3 / 4 \\
3 / 4\end{array}$ & $\begin{array}{l}73 \\
73 \\
68\end{array}$ & $\begin{array}{l}47 \\
47 \\
45\end{array}$ & $\mid \begin{array}{l}20 \\
20 \\
23\end{array}$ & $\begin{array}{l}12 \\
12 \\
14\end{array}$ & $\begin{array}{l}9.0 \\
9.0 \\
9.1\end{array}$ & $\begin{array}{l}6.9 \\
6.9 \\
1.2\end{array}$ & $\begin{array}{l}71 \\
71 \\
14\end{array}$ & & 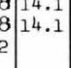 & 2.2 & $\begin{array}{l}2.88 \\
2.88 \\
2.71\end{array}$ & $\begin{array}{l}150.9(\mathrm{~b}) \\
150.9(\mathrm{~b}) \\
128.6(\mathrm{~b})\end{array}$ & 1 & $\begin{array}{l}145 \\
144 \\
127\end{array}$ & $\begin{array}{l}96 \\
95 \\
99\end{array}$ & $\begin{array}{l}0.243 \\
0.248 \\
0.333\end{array}$ & $\begin{array}{l}100 \\
98 \\
100\end{array}$ & $\begin{array}{l}0.092 \\
0.10 \\
-\end{array}$ & \begin{tabular}{|c|}
8.4 \\
8.4 \\
12.3
\end{tabular} & $\mid \begin{array}{l}30.2 \\
34.1 \\
46.1\end{array}$ & $\mid \begin{array}{l}58.0 \\
66.4 \\
81.8\end{array}$ & $\begin{array}{l}2.6 \\
3.0 \\
5.6\end{array}$ & $\begin{array}{l}5.3 \\
6.5 \\
8.8\end{array}$ & $\begin{array}{l}2.04 \\
2.16 \\
1.57\end{array}$ & {$\left[\begin{array}{l}M-H \\
M-H \\
H-V H\end{array}\right.$} & $\begin{array}{l}\mathrm{sc} \\
\text { sc } \\
\text { sc }\end{array}$ \\
\hline & $\begin{array}{l}\text { Westover } \\
\text { Truax }\end{array}$ & & $\begin{array}{l}3 / 4 \\
3 / 4\end{array}$ & $\begin{array}{l}97 \\
90\end{array}$ & $\begin{array}{l}75 \\
79\end{array}$ & $\begin{array}{l}38 \\
28 \\
28\end{array}$ & $\begin{array}{l}14 \\
15\end{array}$ & $\begin{array}{c}7.0 \\
12\end{array}$ & 9.0 & $\begin{array}{l}17 \\
36\end{array}$ & $\begin{array}{l}0.8 \\
4.2\end{array}$ & & & $\begin{array}{l}2.65 \\
2.70\end{array}$ & & 5.6 & $\begin{array}{l}112 \\
130\end{array}$ & $\begin{array}{r}\mathbf{2 9 5} \\
95\end{array}$ & $\begin{array}{l}0.483 \\
0.300\end{array}$ & $\begin{array}{l}97 \\
93\end{array}$ & $=$ & $\left|\begin{array}{l}17.6 \\
10.2\end{array}\right|$ & $\begin{array}{l}73.1 \\
19.5\end{array}$ & $\begin{array}{rr}116.9 \\
23.2\end{array}$ & $\begin{array}{l}4.9 \\
1.5\end{array}$ & $\begin{array}{l}7.4 \\
2.7\end{array}$ & $\begin{array}{l}.51 \\
1.80 \\
80\end{array}$ & H & $\mathrm{T}_{\mathrm{sc}}$ \\
\hline & & & $\begin{array}{l}3 / 4 \\
\frac{1}{2}\end{array}$ & $\begin{array}{l}97 \\
82\end{array}$ & $\begin{array}{l}73 \\
71\end{array}$ & $\begin{array}{l}31 \\
32\end{array}$ & $\begin{array}{l}17 \\
19\end{array}$ & \begin{tabular}{|l|l}
14 \\
13
\end{tabular} & $\begin{array}{c}13 \\
9.4\end{array}$ & $\begin{aligned} 280 \\
50\end{aligned}$ & & & $\begin{array}{l}2.8 \\
2\end{array}$ & $\begin{array}{l}2.73 \\
2.72\end{array}$ & & & $\begin{array}{l}124 \\
129\end{array}$ & $\begin{array}{r}95 \\
>95\end{array}$ & $\begin{array}{l}0.374 \\
0.311 \\
0.311\end{array}$ & $\begin{array}{r}93 \\
100 \\
95\end{array}$ & $\overline{0}$ & $\mid \begin{array}{l}13.6 \\
13.7 \\
10.8\end{array}$ & $\begin{array}{l}53.3 \\
53.3 \\
14.8\end{array}$ & $\mid \begin{array}{r}23.0 \\
18.0 \\
10.8\end{array}$ & $\mid \begin{array}{l}1.3 \\
6.3 \\
2.2\end{array}$ & $\begin{array}{l}1.2 .1 \\
10.2 \\
13.0\end{array}$ & $\begin{array}{l}1.00 \\
.62 \\
.36\end{array}$ & $\begin{array}{l}\mathrm{L} \\
\mathrm{H}-\mathrm{MH} \\
\mathrm{M}\end{array}$ & $\mid \begin{array}{l}\mathrm{SC} \\
\mathrm{T} \\
\mathrm{sc}\end{array}$ \\
\hline & & & $1 \frac{1}{2}$ & 81 & 58 & 33 & 19 & & 6.5 & 56 & & & 0.9 & & $119.0(\mathrm{~d})$ & 15.0 & & & & & & 15.0 & 30.2 & 35.4 & 2.1 & 2.8 & .33 & $m_{M}^{2}$ & $T_{T}^{\infty}$ \\
\hline $\begin{array}{l}\text { TDD-36 } \\
\text { TD-9 }\end{array}$ & $\begin{array}{l}\text { Truax } \\
\text { Truax }\end{array}$ & & $\begin{array}{l}3 / 4 \\
3 / 4\end{array}$ & $\begin{array}{l}82 \\
92\end{array}$ & $\begin{array}{l}71 \\
79 \\
79\end{array}$ & $\begin{array}{l}32 \\
35 \\
35\end{array}$ & $\begin{array}{l}19 \\
22 \\
202\end{array}$ & $\begin{array}{l}13 \\
15 \\
15\end{array}$ & 12.5 & $\begin{array}{l}50 \\
55 \\
55\end{array}$ & & $\begin{array}{l}6 \\
9 \\
94.4 \\
14.4\end{array}$ & $\begin{array}{l}1.6 \\
1.6\end{array}$ & $\begin{array}{l}2.72 \\
2.72 \\
2.72\end{array}$ & $\begin{array}{r}239.0(\mathrm{c}) \\
537.3 \mathrm{~d})\end{array}$ & $\begin{array}{l}5.3 \\
5.6\end{array}$ & $\begin{array}{l}136 \\
130\end{array}$ & $\begin{array}{l}98 \\
95\end{array}$ & $\begin{array}{l}0.246 \\
0.303\end{array}$ & $\begin{array}{l}100 \\
100\end{array}$ & 0.0085 & $\mid \begin{array}{c}9.1 \\
11.1\end{array}$ & $\begin{array}{l}17.2 \\
18.2\end{array}$ & \begin{tabular}{|l|}
$21.0^{\circ}$ \\
22.0
\end{tabular} & $\begin{array}{l}1.8 \\
2.5\end{array}$ & $\begin{array}{l}3.5 \\
2.8\end{array}$ & $\begin{array}{l}1.94 \\
.12\end{array}$ & $\begin{array}{l}\text { L-M } \\
M\end{array}$ & $\begin{array}{l}\text { s-TR } \\
\text { sc }\end{array}$ \\
\hline TD-10 & & & $\begin{array}{l}3 / 4 \\
3 / 4 \\
3 / 4\end{array}$ & \begin{tabular}{|l|}
92 \\
92 \\
02
\end{tabular} & $\begin{array}{l}79 \\
79 \\
70\end{array}$ & $\begin{array}{l}35 \\
35 \\
35\end{array}$ & $\begin{array}{l}22 \\
22 \\
22\end{array}$ & $\begin{array}{l}15 \\
25\end{array}$ & $\begin{array}{l}12 \\
12 \\
12 \\
12\end{array}$ & $\begin{array}{l}55 \\
55 \\
55\end{array}$ & & & $\mid \begin{array}{l}1.6 \\
1.6 \\
1.6\end{array}$ & $\begin{array}{l}2.72 \\
2.72 \\
2.72\end{array}$ & & $\begin{array}{l}5.6 \\
5.6 \\
5.6\end{array}$ & $\begin{array}{l}134 \\
139 \\
132\end{array}$ & $\begin{array}{r}98 \\
102 \\
06\end{array}$ & $\begin{array}{l}0.265 \\
0.216 \\
0.280\end{array}$ & $\begin{array}{r}98 \\
100 \\
100\end{array}$ & & \begin{tabular}{|r|}
9.6 \\
8.0 \\
0.2
\end{tabular} & $\begin{array}{l}14.9 \\
16.3 \\
23.3\end{array}$ & $\mid \begin{array}{l}14.7 \\
15.5 \\
37\end{array}$ & $\left\{\begin{array}{l}2.5 \\
1.3 \\
2.3\end{array}\right.$ & $\begin{array}{l}2.8 \\
1.7 \\
.3\end{array}$ & $\begin{array}{l}1.12 \\
.30 \\
.34\end{array}$ & $\mathrm{~m}$ & $\mid \begin{array}{l}\mathrm{sc} \\
\mathrm{sc}\end{array}$ \\
\hline & & & & & & & & & & & & & CEAY & YEY SILTY & & & & & & & & & & & & & & & \\
\hline $\begin{array}{l}\text { SF-1 } \\
\text { STG-16 } \\
\text { SAFB-7 } \\
\text { TAPB-8 }\end{array}$ & $\begin{array}{l}\text { Sioux Falls } \\
\text { Loring } \\
\text { Thule } \\
\text { Thule }\end{array}$ & SM-SC & $\begin{array}{l}\frac{1}{2} \\
\frac{1}{3} / 4 \\
3 / 4\end{array}$ & $\begin{array}{l}71 \\
87 \\
65 \\
65\end{array}$ & $\begin{array}{l}28 \\
22 \\
39 \\
39\end{array}$ & $\begin{array}{l}16 \\
15 \\
22 \\
22\end{array}$ & $\mid \begin{array}{c}9.0 \\
13 \\
14 \\
14\end{array}$ & $\begin{array}{l}6.0 \\
11 \\
10 \\
10\end{array}$ & $\mid \begin{array}{l}4.3 \\
8.0 \\
7.0 \\
7.0\end{array}$ & $\mid \begin{array}{l}108 \\
260 \\
310 \\
310\end{array}$ & & 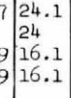 & $\left|\begin{array}{l}5.9 \\
6.0 \\
4.3 \\
4.3\end{array}\right|$ & $\begin{array}{l}2.72 \\
2.72 \\
2.87 \\
2.87\end{array}$ & $\begin{array}{l}\text { r37.0(d) } \\
139.1 \mathrm{~b}) \\
1552.5 \mathrm{~b}) \\
52.5(\mathrm{~b})\end{array}$ & & $\begin{array}{l}131 \\
134 \\
148 \\
146\end{array}$ & $\begin{array}{l}96 \\
96 \\
97 \\
96\end{array}$ & $\left|\begin{array}{l}0.292 \\
0.265 \\
0.215 \\
0.223\end{array}\right|$ & $\begin{array}{l}100 \\
99 \\
100 \\
100\end{array}$ & $\begin{array}{l}0.16 \\
0.12 \\
0.021 \\
0.029\end{array}$ & $\begin{array}{c}10.6 \\
9.7 \\
7.5 \\
7.8\end{array}$ & $\mid \begin{array}{l}15.7 \\
29.1 \\
31.0 \\
35.9 \\
35.9\end{array}$ & $\begin{array}{l}16.6 \\
56.7 \\
61.4 \\
68.8\end{array}$ & $\begin{array}{l}1.7 \\
5.5 \\
2.6 \\
3.3\end{array}$ & $\begin{array}{l}2.7 \\
7.5 \\
4.7 \\
6.5\end{array}$ & $\begin{array}{l}2.58 \\
2.36 \\
. .80 \\
.996\end{array}$ & $\begin{array}{l}\mathrm{L}-\mathrm{M} \\
\mathrm{H}-\mathrm{H} \\
\mathrm{M}-\mathrm{H}\end{array}$ & $\mid \begin{array}{l}\mathrm{sc} \\
\mathrm{sc} \\
\mathrm{sc} \\
\mathrm{sc}\end{array}$ \\
\hline $\mid \begin{array}{c}a A-3 \\
P_{1}-1\end{array}$ & & & $\begin{array}{l}1 \frac{1}{\frac{1}{2}} \\
1 \frac{\frac{1}{3}}{3 / 4} \\
3 / 4\end{array}$ & $\begin{array}{l}91 \\
62 \\
98 \\
98\end{array}$ & $\begin{array}{l}48 \\
33 \\
62 \\
68\end{array}$ & $\begin{array}{l}23 \\
22 \\
21 \\
29\end{array}$ & $\begin{array}{l}15 \\
15 \\
16 \\
18\end{array}$ & $\begin{array}{l}13 \\
10 \\
14 \\
16\end{array}$ & $\left|\begin{array}{l}11 \\
5.5 \\
12 \\
14\end{array}\right|$ & $\begin{array}{l}225 \\
400 \\
137 \\
195\end{array}$ & $\begin{array}{l}13 \\
2.7 \\
14 \\
11\end{array}$ & $7 \begin{array}{l}22.0 \\
22.0 \\
21.8 \\
22.0\end{array} \mid$ & $\begin{array}{l}4.6 \\
6.1 \\
6.0 \\
4.6\end{array}$ & $\begin{array}{l}2.64 \\
2.74 \\
2.65 \\
2.65\end{array}$ & $\begin{array}{r}220.8(d) \\
-8(d) \\
220.8(d)\end{array}$ & $\begin{array}{l}7.2 \\
7.2 \\
7.2\end{array}$ & $\begin{array}{l}120 \\
115 \\
118 \\
119\end{array}$ & $\begin{array}{l}99 \\
95 \\
98 \\
99\end{array}$ & $\mid \begin{array}{l}0.378 \\
0.267 \\
0.403 \\
0.393\end{array}$ & $\begin{array}{l}100 \\
100 \\
100 \\
05\end{array}$ & $\begin{array}{l}0.66 \\
0.001 \\
1.3 \\
1.52\end{array}$ & $\mid \begin{array}{l}14.2 \\
9.7 \\
15.2 \\
15.2\end{array}$ & $\begin{array}{l}22.6 \\
26.0 \\
212.7 \\
21.7\end{array}$ & 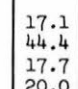 & $\begin{array}{l}1.5 \\
3.3 \\
1.6 \\
2.6\end{array}$ & $\begin{array}{l}2.7 \\
4.2 \\
2.3 \\
3.2\end{array}$ & $\begin{array}{l}2.80 \\
2.27 \\
. .44\end{array}$ & $\mid \begin{array}{l}L-M \\
M-H \\
L-M\end{array}$ & $\mid \begin{array}{l}\mathrm{sc} \\
\mathrm{sc} \\
\mathrm{sc} \\
\mathrm{sc}\end{array}$ \\
\hline & & & $1 \frac{1}{2}$ & & & & & 15 & 10 & & & & 5.1 & & & - & & & & & & & & 23.1 & $\begin{array}{l}2.6 \\
1.3\end{array}$ & 2.0 & & I & $\mathrm{T}$ \\
\hline 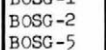 & & & $1 \frac{4}{1 \frac{5}{2}}$ & $\begin{array}{l}94 \\
94\end{array}$ & $\begin{array}{l}75 \\
75\end{array}$ & $\begin{array}{l}44 \\
44\end{array}$ & $\begin{array}{l}21 \\
21\end{array}$ & 15 & $\begin{array}{l}10 \\
10\end{array}$ & 33 & & & & $\begin{array}{l}2.76 \\
2.76\end{array}$ & $\begin{array}{l}339.5(\mathrm{c}) \\
339.5(\mathrm{c})\end{array}$ & & $\begin{array}{l}136 \\
134\end{array}$ & $\begin{array}{l}97 \\
96\end{array}$ & $\begin{array}{l}0.267 \\
0.282\end{array}$ & $\begin{array}{l}100 \\
100\end{array}$ & $\begin{array}{l}0.0001 \\
0.0002\end{array}$ & \begin{tabular}{|c|}
9.7 \\
10.2
\end{tabular} & $\begin{array}{l}12.8 \\
16.2\end{array}$ & .8 & $\begin{array}{l}1.3 \\
1.7\end{array}$ & $\begin{array}{l}1.7 \\
3.7\end{array}$ & $\begin{array}{l}2.30 \\
2.18\end{array}$ & L-M & $\mid \begin{array}{l}\mathbf{T} \\
T\end{array}$ \\
\hline LST-3 & & & $\begin{array}{l}1 \frac{1}{2} \\
1 \frac{1}{2}\end{array}$ & \begin{tabular}{|l}
83 \\
87
\end{tabular} & 62 & 48 & $\begin{array}{ll}30 \\
32\end{array}$ & & 15 & & & & & $\begin{array}{l}2.71 \\
2.71\end{array}$ & & & $\begin{array}{l}127 \\
127\end{array}$ & $>95$ & $|0.334|$ & $\begin{array}{l}100 \\
100\end{array}$ & $\begin{array}{l}0.0034 \\
0.0034\end{array}$ & 12.3 & 50.1 & 56.9 & 2.8 & 3.3 & $\begin{array}{l}2.30 \\
.18\end{array}$ & & $\mid \begin{array}{l}\mathrm{Sc} \\
\mathrm{sc}\end{array}$ \\
\hline
\end{tabular}


Table AI (Cont'd).

\begin{tabular}{|c|c|c|c|c|c|c|c|c|c|c|c|c|c|c|c|c|c|c|c|c|c|c|c|c|c|c|c|c|c|}
\hline \multirow{3}{*}{$\begin{array}{l}\text { Specimen } \\
\text { Number }\end{array}$} & \multirow{3}{*}{ Material Source } & \multicolumn{10}{|c|}{ SOIL GRADATION DATA (As Frozen) } & \multicolumn{5}{|c|}{ PHYSICAL PROPERTIES OF BASIC SOIL } & \multicolumn{5}{|c|}{ SṔECIMEN DATÁ (As Mòlded) } & \multicolumn{7}{|c|}{ FREEZING TEST DATA } & \multirow{3}{*}{$\begin{array}{l}\text { Typ } \\
\text { of } \\
\text { Cyl. } \\
\text { (12) }\end{array}$} \\
\hline & & \multirow{2}{*}{$\begin{array}{l}\text { Unified } \\
\text { Soil } \\
\text { clossifi- } \\
\text { cotion } \\
\text { Symbol" } \\
\text { (2) }\end{array}$} & \multirow{2}{*}{\begin{tabular}{|l} 
Moxi- \\
mum \\
Size \\
in.
\end{tabular}} & \multicolumn{6}{|c|}{ Percent finer, $\mathrm{mm}$} & \multicolumn{2}{|c|}{$\begin{array}{c}\text { Coef ficients } \\
(3)\end{array}$} & \multicolumn{2}{|c|}{$\begin{array}{l}\text { Atterberg } \\
\text { Limits (4) }\end{array}$} & \multirow{2}{*}{$\begin{array}{l}\text { Specific } \\
\text { Grovity }\end{array}$} & \multicolumn{2}{|c|}{$\underset{(5)}{\text { Compaction Dato }}$} & \multirow{2}{*}{$\begin{array}{c}\text { Dry } \\
\text { Unit } \\
\text { Weight } \\
\text { pcf }\end{array}$} & \multirow{2}{*}{$\begin{array}{c}\text { Degree } \\
\text { of } \\
\text { Compac- } \\
\text { tion } \\
\\
\%\end{array}$} & \multirow{2}{*}{$\begin{array}{c}\text { Void } \\
\text { Rotio } \\
\%\end{array}$} & \multirow{2}{*}{$\begin{array}{c}\text { G, ot } \\
\text { Stort } \\
\text { of } \\
\text { Test } \\
(6) \\
\%\end{array}$} & \multirow{2}{*}{$\begin{array}{c}\text { Porme- } \\
\text { obilify } \\
k \\
(7) \\
\mathrm{cm} / \mathrm{sec} \\
\times 10^{-4}\end{array}$} & \multicolumn{2}{|c|}{$\begin{array}{c}\text { Avg. } \\
\text { woter } \\
\text { Content }\end{array}$} & \multirow{2}{*}{\begin{tabular}{|c|} 
\\
Total \\
Heave \\
$(8)$ \\
$\%$
\end{tabular}} & \multicolumn{2}{|c|}{\begin{tabular}{|c|}
$\begin{array}{c}\text { Rate of } \\
\text { Heove } \\
\text { mm /day ( } 9)\end{array}$ \\
\end{tabular}} & \multirow{2}{*}{\begin{tabular}{|l|} 
Heave \\
Rate \\
vor. \\
Index \\
$(10)$
\end{tabular}} & \multirow{2}{*}{$\begin{array}{l}\text { Frost } \\
\text { Susc } \\
\text { Clos: } \\
\text { (III) }\end{array}$} & \\
\hline & & & & 4.76 & 0.42 & 0.074 & 0.02 & 0.01 & 0.005 & $c_{u}$ & $c_{c}$ & LL & PI & & $\left|\begin{array}{c}\text { Moximumum } \\
\text { Dry Unif } \\
\text { weight } \\
\text { pcf }\end{array}\right|$ & $\begin{array}{c}\text { Optimum } \\
\text { Moisture } \\
\text { Content } \\
\%\end{array}$ & & & & & & \begin{tabular}{|c|} 
Before \\
Test \\
$\%$
\end{tabular} & $\begin{array}{c}\text { After } \\
\text { Tost } \\
\%\end{array}$ & & Avg. & Mox. & & & \\
\hline $\begin{array}{l}\text { FA-1 } \\
\text { FA-4 } \\
\text { PBJ-1 } \\
\text { BH-1A }\end{array}$ & $\begin{array}{l}\text { Fargo } \\
\text { Fargo } \\
\text { Proj. Blue Jay } \\
\text { Breed's H1ll (EBT) }\end{array}$ & $\mathrm{sc}$ & $\begin{array}{l}3 / 4 \\
3 / 4 \\
3 / 4 \\
3 / 4\end{array}$ & $\begin{array}{l}98 \\
98 \\
73 \\
76\end{array}$ & $\begin{array}{l}33 \\
33 \\
55 \\
60\end{array}$ & $\begin{array}{l}17 \\
17 \\
35 \\
41\end{array}$ & $\begin{array}{l}9.5 \\
9.5 \\
23 \\
24\end{array}$ & $\begin{array}{l}7.5 \\
7.5 \\
20 \\
-\end{array}$ & $\begin{array}{c}5.5 \\
5.5 \\
15 \\
-\end{array}$ & \begin{tabular}{|l}
50 \\
50 \\
500 \\
191
\end{tabular} & $\begin{array}{l}5.2 \\
5.2 \\
1.7 \\
1.1\end{array}$ & $\begin{array}{l}30.7 \\
30.7 \\
24.7 \\
2 \div .0\end{array}$ & $\begin{array}{r}10.5 \\
10.5 \\
8.1 \\
11.0\end{array}$ & \begin{tabular}{|l|} 
CLAYEY SA \\
2.70 \\
2.70 \\
2.70 \\
2.75
\end{tabular} & $\begin{array}{l}127.2(d) \\
127.2(d) \\
133.1(c) \\
138.7(c)\end{array} \mid$ & $\begin{array}{l}9.0 \\
9.0 \\
9.8 \\
7.2\end{array}$ & $\begin{array}{l}123 \\
118 \\
134 \\
139\end{array}$ & $\begin{array}{r}97 \\
93 \\
101 \\
100\end{array}$ & $\mid \begin{array}{l}0.374 \\
0.424 \\
0.272 \\
0.237\end{array}$ & $\begin{array}{r}100 \\
100 \\
100 \\
94\end{array}$ & $\begin{array}{l}0.09 \\
0.17 \\
0.033 \\
-\end{array}$ & $\begin{array}{r}13.9 \\
15.7 \\
8.0 \\
8.0\end{array}$ & $\begin{array}{l}21.5 \\
32.8 \\
17.9 \\
10.5\end{array}$ & $\begin{array}{r}18.7 \\
42.4 \\
25.3 \\
7.3\end{array}$ & $\begin{array}{l}1.5 \\
3.3 \\
2.2 \\
0.6\end{array}$ & $\begin{array}{l}2.7 \\
4.5 \\
2.8 \\
1.0\end{array}$ & $\begin{array}{l}1.80 \\
1.36 \\
1.27 \\
1.66\end{array}$ & $\begin{array}{l}\mathrm{L}-\mathrm{M} \\
\mathrm{M}-\mathrm{H} \\
\mathrm{M} \\
\mathrm{VL}\end{array}$ & $\begin{array}{l}\mathrm{SC} \\
\mathrm{SC} \\
\mathrm{SC} \\
\mathrm{SL}\end{array}$ \\
\hline $\begin{array}{l}\text { W0-9 } \\
\text { MIN-7 } \\
\text { PBJ-15 } \\
\text { PBJ-16 } \\
\end{array}$ & $\begin{array}{l}\text { Westover } \\
\text { Minnesota } \\
\text { Proj. Blue Jay } \\
\text { Proj. Blue Jay }\end{array}$ & & $\begin{array}{l}3 \\
3 / 4 \\
3 / 4 \\
3 / 4 \\
\end{array}$ & $\begin{array}{l}82 \\
97 \\
80 \\
80 \\
\end{array}$ & \begin{tabular}{|l}
66 \\
78 \\
58 \\
58 \\
\end{tabular} & $\begin{array}{l}48 \\
48 \\
44 \\
44 \\
\end{array}$ & $\begin{array}{l}30 \\
31 \\
35 \\
35 \\
\end{array}$ & $\begin{array}{l}23 \\
31 \\
31 \\
31\end{array}$ & $\begin{array}{l}17 \\
22 \\
22\end{array}$ & $\begin{array}{l}115 \\
310 \\
310 \\
\end{array}$ & $\begin{array}{l}0.9 \\
- \\
0.1 \\
0.1 \\
\end{array}$ & \begin{tabular}{|l|}
20.7 \\
28.7 \\
18.6 \\
18.6
\end{tabular} & $\begin{array}{r}7.2 \\
10.7 \\
9.2 \\
9.2 \\
\end{array}$ & $\begin{array}{l}2.71 \\
2.70 \\
2.75 \\
2.75 \\
\end{array}$ & $\left|\begin{array}{c}- \\
139.6(\mathrm{c}) \\
139.6(\mathrm{c})\end{array}\right|$ & $\begin{array}{c}- \\
- \\
7.0 \\
7.0\end{array}$ & $\begin{array}{l}130 \\
114 \\
139 \\
132 \\
\end{array}$ & $\begin{array}{r}>95 \\
>95 \\
100 \\
95 \\
\end{array}$ & $\begin{array}{l}0.297 \\
0.478 \\
0.234 \\
0.301 \\
\end{array}$ & $\begin{array}{r}100 \\
91 \\
100 \\
100 \\
\end{array}$ & \begin{tabular}{c|} 
\\
- \\
0.0027 \\
0.0042 \\
\end{tabular} & \begin{tabular}{|r|}
10.9 \\
16.2 \\
8.5 \\
10.9 \\
\end{tabular} & \begin{tabular}{|l|}
22.3 \\
32.0 \\
17.3 \\
34.7 \\
\end{tabular} & $\begin{array}{l}31.5 \\
38.6 \\
26.3 \\
83.0 \\
\end{array}$ & \begin{tabular}{|l|}
3.1 \\
1.8 \\
2.2 \\
4.6 \\
\end{tabular} & \begin{tabular}{|l|}
4.6 \\
2.5 \\
3.8 \\
8.3 \\
\end{tabular} & $\begin{array}{l}1.48 \\
1.38 \\
1.72 \\
1.80 \\
\end{array}$ & $\begin{array}{l}M-H \\
L-M \\
M \\
H-V H \\
\end{array}$ & S \\
\hline 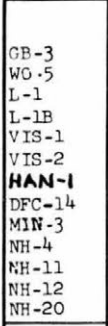 & $\begin{array}{l}\text { Goose Bay } \\
\text { Westover } \\
\text { Labrador } \\
\text { Labrador } \\
\text { Valpara:so } \\
\text { Valparaiso } \\
\text { Hanover } \\
\text { Dow Field } \\
\text { Minnesota } \\
\text { New Hampshire } \\
\text { New Hampshire } \\
\text { New Hampshire } \\
\text { New Hampshire }\end{array}$ & $\mathrm{ML}$ & $\begin{array}{c}- \\
- \\
- \\
- \\
- \\
3 / 4 \\
3 / 4 \\
= \\
- \\
-\end{array}$ & $\begin{array}{l}100 \\
100 \\
1100 \\
1: 0 \\
100 \\
100 \\
100 \\
95 \\
97 \\
100 \\
100 \\
100 \\
100 \\
\end{array}$ & \begin{tabular}{|l}
99 \\
91 \\
200 \\
010 \\
100 \\
100 \\
100 \\
91 \\
92 \\
99 \\
99 \\
99 \\
99 \\
\end{tabular} & $\begin{array}{l}5 ! \\
53 \\
95 \\
95 \\
99 \\
99 \\
98 \\
87 \\
83 \\
97 \\
97 \\
97 \\
97 \\
\end{array}$ & \begin{tabular}{|l|}
6.0 \\
13 \\
.27 \\
27 \\
54 \\
54 \\
35 \\
54 \\
63 \\
60 \\
50 \\
60 \\
60 \\
\end{tabular} & $\begin{array}{r}- \\
10 \\
10 \\
25 \\
25 \\
18 \\
40 \\
44 \\
22 \\
22 \\
22 \\
22\end{array}$ & $\begin{array}{l}- \\
- \\
- \\
15 \\
15 \\
8 \\
28 \\
28 \\
10 \\
10 \\
10 \\
10\end{array}$ & $\begin{array}{l}- \\
\vdots \\
\vdots \\
\vdots \\
\vdots \\
\vdots \\
\vdots \\
-\end{array}$ & $\begin{array}{l}- \\
- \\
- \\
- \\
- \\
- \\
- \\
= \\
- \\
-\end{array}$ & \begin{tabular}{|l|}
26.0 \\
25.0 \\
23.7 \\
23.7 \\
29.5 \\
32.8 \\
36.0 \\
26.6 \\
26.6 \\
26.6 \\
26.6
\end{tabular} & \begin{tabular}{|c|}
3.0 \\
3.0 \\
4.0 \\
4.0 \\
12.7 \\
8.1 \\
5.1 \\
0.1 \\
0.1 \\
0.1 \\
0.1 \\
\end{tabular} & \begin{tabular}{|l|} 
AND SAND \\
2.74 \\
2.6 \\
2.77 \\
2.77 \\
2.72 \\
2.72 \\
2.75 \\
2.66 \\
2.62 \\
2.70 \\
2.70 \\
2.70 \\
2.70 \\
\end{tabular} & $\begin{array}{l}\text { DY SILTS } \\
\left|\begin{array}{l|}102.0(\mathrm{c}) \\
113.6(\mathrm{~d}) \\
102.0(\mathrm{~d}) \\
102.0 \mathrm{~d}) \\
115.8(\mathrm{~d}) \\
115.8 \mathrm{~d}) \\
105.6(\mathrm{~d}) \\
107.1(\mathrm{c}) \\
116.7(\mathrm{c}) \\
106.7 \mathrm{c}) \\
106.7(\mathrm{c}) \\
106.7 \mathrm{c})\end{array}\right|\end{array}$ & $\begin{array}{r}7.9 \\
11.0 \\
18.1 \\
18.1 \\
13.5 \\
13.5 \\
16.9 \\
16.9 \\
16.5 \\
16.5 \\
16.5 \\
10.5 \\
\end{array}$ & $\begin{array}{l}102 \\
112 \\
106 \\
103 \\
113 \\
113 \\
101 \\
104 \\
101 \\
106 \\
108 \\
105 \\
\end{array}$ & $\begin{array}{r}100 \\
99 \\
104 \\
102 \\
98 \\
98 \\
96 \\
98 \\
>95 \\
98 \\
98 \\
101 \\
98 \\
\end{array}$ & \begin{tabular}{|l|}
0.688 \\
0.484 \\
0.626 \\
0.668 \\
0.501 \\
0.501 \\
0.695 \\
0.590 \\
0.611 \\
8.589 \\
0.567 \\
0.611 \\
\end{tabular} & \begin{tabular}{|c|}
100 \\
100 \\
100 \\
94 \\
98 \\
100 \\
100 \\
100 \\
99 \\
100 \\
100 \\
100 \\
100 \\
\end{tabular} & \begin{tabular}{|c|}
3.6 \\
- \\
0.4 \\
0.5 \\
0.024 \\
0.024 \\
- \\
0.000004 \\
- \\
0.13 \\
0.16 \\
0.11 \\
0.15 \\
\end{tabular} & \begin{tabular}{|l|l}
24.4 & \\
18.0 & 2 \\
22.7 & 2 \\
22.4 & 2 \\
18.4 & 6 \\
18.0 & 6 \\
28.8 & 1 \\
22.2 & \\
22.2 & \\
23.0 & \\
21.7 \\
22.8 \\
20.9 & \\
22.6 & \\
22.6 &
\end{tabular} & \begin{tabular}{|r|r}
25.6 \\
26.0 \\
27.3 \\
27.3 \\
30.0 \\
62.1 \\
65.6 \\
79.0 \\
136.8 \\
130.6 \\
50.6 \\
128.6 \\
228.7 \\
99.9 \\
116.7 & 1 \\
\end{tabular} & \begin{tabular}{|r|}
7.0 \\
17.3 \\
11.4 \\
16.3 \\
95.3 \\
100.0 \\
105.6 \\
104.0 \\
84.6 \\
126.2 \\
130.1 \\
190.6 \\
239.2 \\
\end{tabular} & $\begin{array}{r}0.3 \\
1.0 \\
1.2 \\
1.5 \\
2.8 \\
10.0 \\
14.1 \\
13.9 \\
3.5 \\
15: 9 \\
15.9 \\
26.0 \\
12.8 \\
\end{array}$ & \begin{tabular}{r|}
1.0 \\
1.6 \\
1.5 \\
2.3 \\
11.5 \\
1.3 .3 \\
16.8 \\
23.3 \\
5.8 \\
20.5 \\
18.0 \\
28.3 \\
19.7 \\
\end{tabular} & $\begin{array}{l}3.33 \\
1.50 \\
1.25 \\
1.53 \\
1.17 \\
1.33 \\
1.19 \\
1.68 \\
1.66 \\
1.30 \\
1.13 \\
1.08 \\
1.54 \\
\end{array}$ & $\begin{array}{l}\mathrm{N}-\mathrm{VI} \\
\mathrm{L} \\
\mathrm{L} \\
\mathrm{L}-\mathrm{M} \\
\mathrm{L} H \\
\mathrm{VH} \\
\mathrm{VH} \\
\mathbf{Y H} \\
\mathrm{VH} \\
\mathrm{YH}-\mathrm{H} \\
\mathrm{VHH} \\
\mathrm{VH} \\
\mathrm{VH} \\
\mathrm{VH} \\
\end{array}$ & \begin{tabular}{|l} 
SC \\
SC \\
$S C$ \\
$S C$ \\
$S C$ \\
$S C$ \\
SC \\
$T$ \\
$S C$ \\
$S T$ \\
$S C$ \\
$S C$ \\
$S C$ \\
$S C$ \\
\end{tabular} \\
\hline $\begin{array}{l}\text { YS-1 } \\
\text { NH-79A } \\
\text { NH-31 }\end{array}$ & $\begin{array}{l}\text { Yukon } \\
\text { New Hampshire } \\
\text { llew Hamnehire }\end{array}$ & ML-CL & $\overline{-}$ & $\begin{array}{l}100 \\
100 \\
100\end{array}$ & $\begin{array}{l}100 \\
100 \\
96\end{array}$ & $\begin{array}{l}98 \\
86 \\
90\end{array}$ & $\begin{array}{l}60 \\
61 \\
67\end{array}$ & $\begin{array}{l}37 \\
34 \\
36\end{array}$ & $\begin{array}{l}22 \\
14 \\
16\end{array}$ & 5 & $\overline{-}$ & $\begin{array}{l}25.3 \\
24.1 \\
25.0\end{array}$ & $\begin{array}{r}\mathrm{C} \\
.8 \\
.9 \\
.0\end{array}$ & \begin{tabular}{|l|} 
CLAYEY SI \\
2.73 \\
2.76 \\
2.70 \\
\end{tabular} & 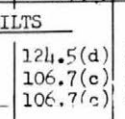 & $\begin{array}{l}11.5 \\
16.5 \\
10.5\end{array}$ & $\begin{array}{l}123 \\
105 \\
102\end{array}$ & $\begin{array}{l}99 \\
98 \\
95\end{array}$ & \begin{tabular}{|l|}
0.389 \\
0.643 \\
0.662
\end{tabular} & $\begin{array}{r}100 \\
88 \\
100\end{array}$ & $\begin{array}{l}0.0031 \\
0.054 \\
0.033\end{array}$ & $\begin{array}{l}14.2 \\
20.5 \\
214.5\end{array}$ & \begin{tabular}{l|l}
28.9 \\
78.1 \\
84.6
\end{tabular} & \begin{tabular}{|}
37.0 \\
150.2 \\
117.6
\end{tabular} & $\begin{array}{r}2.2 \\
7.9 \\
14.0\end{array}$ & $\begin{array}{r}3.5 \\
15.8 \\
18.3\end{array}$ & $\begin{array}{l}1.52 \\
2.00 \\
1.30\end{array}$ & $\mid \begin{array}{l}\mathrm{M} \\
\mathrm{H}-\mathrm{VH} \\
\mathrm{VH}\end{array}$ & $\begin{array}{l}\mathrm{SC} \\
\mathrm{SC} \\
\mathrm{SC}\end{array}$ \\
\hline $\begin{array}{l}\mathrm{NH}-29 \mathrm{~A} \\
\mathrm{NH}-44 \mathrm{~A} \\
\mathrm{NH}-49 \mathrm{~A}\end{array}$ & $\begin{array}{l}\text { New Hampshire } \\
\text { New Hampshire } \\
\text { New Hampshire }\end{array}$ & & - & $\begin{array}{l}100 \\
100 \\
100 \\
\end{array}$ & $\begin{array}{l}93 \\
100 \\
100\end{array}$ & $\begin{array}{l}85 \\
99 \\
99\end{array}$ & $\begin{array}{l}73 \\
73 \\
73 \\
\end{array}$ & $\begin{array}{l}47 \\
37 \\
37\end{array}$ & $\begin{array}{l}23 \\
13 \\
13\end{array}$ & : & 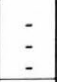 & \begin{tabular}{|l|}
26.0 \\
23.7 \\
23.7
\end{tabular} & \begin{tabular}{|l|}
5.0 \\
6.0 \\
6.0
\end{tabular} & $\begin{array}{l}2.70 \\
2.70 \\
2.70 \\
\end{array}$ & $\left|\begin{array}{l}106.7(c) \\
110.1(c) \\
110.1(c)\end{array}\right|$ & $\begin{array}{l}16.5 \\
14.7 \\
14.7 \\
\end{array}$ & $\begin{array}{l}101 \\
107 \\
106\end{array}$ & $\begin{array}{l}95 \\
97 \\
96\end{array}$ & $\begin{array}{l}0.674 \\
0.577 \\
0.596\end{array}$ & $\begin{array}{r}100 \\
99 \\
100 \\
\end{array}$ & $\begin{array}{c}0.036 \\
- \\
\end{array}$ & $\begin{array}{l}25.0 \\
21.2 \\
22.0 \\
22.0\end{array}$ & $\begin{array}{l}86.8 \\
42.4 \\
36.4\end{array}$ & \begin{tabular}{|r|}
235.3 \\
50.8 \\
29.8 \\
\end{tabular} & $\begin{array}{r}14.0 \\
3.7 \\
4.0 \\
\end{array}$ & $\begin{array}{r}15.5 \\
4.5 \\
5.3\end{array}$ & $\begin{array}{l}1.10 \\
1.22 \\
1.32 \\
\end{array}$ & $\begin{array}{l}\mathrm{VH} \\
\mathrm{M}-\mathrm{H} \\
\mathrm{H}\end{array}$ & $\begin{array}{l}\mathrm{SC} \\
\mathrm{SC} \\
\mathrm{SM} \\
\end{array}$ \\
\hline $\begin{array}{l}\text { LF -4 } \\
\text { LFT }-13 \\
\text { LFT }-14 \\
\end{array}$ & $\begin{array}{l}\text { Ladd Field } \\
\text { Fa1rbanks } \\
\text { Fairbanks }\end{array}$ & ML-OL & - & $\begin{array}{l}1: 0 \\
100 \\
100 \\
\end{array}$ & $\begin{array}{l}100 \\
100 \\
100\end{array}$ & $\begin{array}{l}91 \\
97 \\
97 \\
\end{array}$ & $\begin{array}{l}38 \\
42 \\
42 \\
\end{array}$ & $\begin{array}{l}13 \\
22 \\
22 \\
\end{array}$ & $\begin{array}{l}6.0 \\
12 \\
12 \\
\end{array}$ & 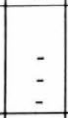 & 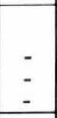 & $\begin{array}{l}31.0 \\
32.6 \\
32.6\end{array}$ & \begin{tabular}{|c|} 
\\
\\
\\
.2 \\
6.2 \\
\end{tabular} & \begin{tabular}{|c|} 
S WITH OR \\
2.75 \\
2.67 \\
2.57 \\
\end{tabular} & $\begin{array}{l}101.6(\mathrm{~d}) \\
107.4(\mathrm{c}) \\
107.4(\mathrm{c})\end{array}$ & $\begin{array}{l}18.1 \\
17.1 \\
17.1 \\
\end{array}$ & $\begin{array}{r}98 \\
101 \\
111 \\
\end{array}$ & $\begin{array}{r}97 \\
95 \\
103 \\
\end{array}$ & \begin{tabular}{|l|}
0.737 \\
0.646 \\
0.505 \\
\end{tabular} & $\begin{array}{l}100 \\
100 \\
100 \\
\end{array}$ & $\begin{array}{l}0.64 \\
0.20 \\
0.09\end{array}$ & \begin{tabular}{|l|}
26.8 \\
24.2 \\
18.9
\end{tabular}. & \begin{tabular}{r|r}
45.7 \\
112.6 \\
105.7
\end{tabular} & \begin{tabular}{|r|}
36.5 \\
223.6 \\
281.2 \\
281.2
\end{tabular} & $\begin{array}{r}3.1 \\
11.3 \\
11.5 \\
\end{array}$ & $\begin{array}{r}4.0 \\
14.0 \\
13.7\end{array}$ & \begin{tabular}{|l|}
1.27 \\
1.24 \\
1.19 \\
\end{tabular} & $\begin{array}{l}M \\
v H \\
v H \\
y_{H} \\
\end{array}$ & $\begin{array}{l}\mathrm{SC} \\
\mathrm{sc} \\
\mathrm{SC} \\
\end{array}$ \\
\hline $\begin{array}{l}\text { DFT - } 5 \\
\text { FB-20A } \\
\text { EBT-3 }-3 \% \\
\text { EBT - } 4 * \\
\text { EBT }-21\end{array}$ & $\begin{array}{l}\text { Dow Field } \\
\text { Fort Belvoir } \\
\text { East Boston } \\
\text { East Boston } \\
\text { East Boston }\end{array}$ & $\mathrm{CL}$ & $\begin{array}{l}3 / 4 \\
3 / 4 \\
3 / 4 \\
3 / 4 \\
3 / 4\end{array}$ & $\begin{array}{l}82 \\
95 \\
.84 \\
84 \\
84\end{array}$ & $\begin{array}{l}70 \\
87 \\
72 \\
72 \\
72\end{array}$ & $\mid \begin{array}{l}62 \\
64 \\
56 \\
56 \\
56\end{array}$ & $\begin{array}{l}40 \\
43 \\
43 \\
43 \\
43\end{array}$ & $\begin{array}{l}31 \\
36 \\
35 \\
35 \\
35\end{array}$ & $\begin{array}{l}23 \\
30 \\
25 \\
25 \\
25\end{array}$ & $\begin{array}{l}: \\
:\end{array}$ & $\begin{array}{l}- \\
- \\
- \\
- \\
-\end{array}$ & $\begin{array}{r}\quad \mathrm{GH} \\
25.6 \\
41.0 \\
23.0 \\
23.0 \\
23.0\end{array}$ & \begin{tabular}{|r|}
$\mid 7.9$ \\
8.0 \\
7.0 \\
7.0 \\
7.0
\end{tabular} & \begin{tabular}{|l|} 
LIX AND SA \\
2.73 \\
2.70 \\
2.76 \\
2.76 \\
2.76
\end{tabular} & 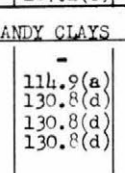 & 15.6 & $\begin{array}{l}133 \\
115 \\
126 \\
130 \\
125\end{array}$ & $\begin{array}{r}>95 \\
100 \\
96 \\
99 \\
96\end{array}$ & \begin{tabular}{|l|}
0.352 \\
0.468 \\
0.371 \\
0.324 \\
0.374
\end{tabular} & $\begin{array}{r}100 \\
94 \\
100 \\
100 \\
100\end{array}$ & $\begin{array}{l}0.0009 \\
0.0003 \\
0.0010\end{array}$ & $\begin{array}{l}12.8 \\
2: .3 \\
23.3 \\
13.4 \\
11.7 \\
13.6\end{array}$ & \begin{tabular}{l|}
42.7 \\
25.1 \\
46.5 \\
30.2 \\
22.9
\end{tabular} & \begin{tabular}{|r|}
73.0 \\
25.0 \\
95.3 \\
47.7 \\
122.9
\end{tabular} & $\begin{array}{l}4.8 \\
1.3 \\
5.5 \\
4.0 \\
7.0\end{array}$ & $\mid \begin{array}{r}10.3 \\
2.0 \\
10.5 \\
5.5 \\
11.1\end{array}$ & $\begin{array}{l}2.14 \\
1.54 \\
1.52 \\
1.38 \\
1.58\end{array}$ & $\begin{array}{l}\mathrm{H}-\mathrm{VH} \\
\mathrm{U} \\
\mathrm{H}-\mathrm{VH} \\
\mathrm{H} \\
\mathrm{H}-\mathrm{VH}\end{array}$ & \begin{tabular}{l|l}
$H$ & $S C$ \\
SL \\
SC \\
SC \\
SC \\
SC
\end{tabular} \\
\hline $\begin{array}{l}E B T-22 \\
F B-1 A \\
F B-7 A \\
F B-7 A \\
F B-14 A\end{array}$ & $\begin{array}{l}\text { East Boston } \\
\text { Fort Belvoir } \\
\text { Fort Belvoir } \\
\text { Fort Belvoir } \\
\text { Fort Pelvoir }\end{array}$ & & $\begin{array}{l}3 / 4 \\
1 / 4 \\
1 / 4 \\
1 / 4 \\
1 / 4\end{array}$ & $\begin{array}{l}84 \\
98 \\
98 \\
98 \\
98\end{array}$ & $\begin{array}{l}72 \\
90 \\
90 \\
90 \\
98\end{array}$ & $\begin{array}{l}56 \\
61 \\
61 \\
61 \\
61\end{array}$ & $\begin{array}{l}43 \\
49 \\
49 \\
49 \\
49\end{array}$ & $\begin{array}{l}35 \\
41 \\
41 \\
41 \\
41\end{array}$ & $\begin{array}{l}25 \\
34 \\
34 \\
34 \\
34\end{array}$ & $\begin{array}{l}- \\
- \\
- \\
-\end{array}$ & $\begin{array}{l}- \\
- \\
- \\
-\end{array}$ & \begin{tabular}{|l|l|}
23.0 \\
43.8 \\
43.8 \\
43.8 \\
43.8
\end{tabular} & \begin{tabular}{|l|}
7.0 \\
20.3 \\
20.3 \\
20.3 \\
20.3
\end{tabular} & $\begin{array}{l}2.75 \\
2.73 \\
2.73 \\
2.73 \\
2.73\end{array}$ & $\begin{array}{l}130.8(\mathrm{~d}) \\
114.9(\mathrm{a}) \\
1114.9(\mathrm{a}) \\
1114.9(\mathrm{a}) \\
114.9(\mathrm{a})\end{array}$ & $\begin{array}{l}15.6 \\
15.6 \\
15.6 \\
15.6\end{array}$ & $\begin{array}{l}130 \\
110 \\
117 \\
113 \\
118\end{array}$ & $\begin{array}{r}99 \\
96 \\
102 \\
98 \\
103\end{array}$ & $\begin{array}{l}0.328 \\
0.536 \\
0.456 \\
0.504 \\
0.441\end{array}$ & $\begin{array}{l}100 \\
100 \\
90 \\
100 \\
100\end{array}$ & $\begin{array}{l}0.0002 \\
0.148 \\
0.098 \\
0.130 \\
0.083\end{array}$ & $\begin{array}{l}11.9 \\
19.8 \\
15.0 \\
18.5 \\
16.2\end{array}$ & \begin{tabular}{|l|}
34.9 \\
81.5 \\
22.3 \\
27.4 \\
27.0
\end{tabular} \mid & \begin{tabular}{|r|}
81.1 \\
188.4 \\
18.2 \\
22.1 \\
27.6
\end{tabular} & $\begin{array}{l}.6 .5 \\
7.7 \\
1.5 \\
1.3 \\
2.2\end{array}$ & $\begin{array}{r}7.5 \\
12.0 \\
3.7 \\
3.0 \\
3.2\end{array}$ & $\begin{array}{l}1.15 \\
1.56 \\
2.45 \\
2.30 \\
1.45\end{array}$ & $\begin{array}{l}\text { H } \\
\mathrm{VH} \\
\mathrm{L}-\mathrm{H} \\
\mathrm{t}-\mathrm{y} \\
\mathrm{M}\end{array}$ & $\begin{array}{l}\mathrm{SC} \\
\mathrm{SC} \\
3 \mathrm{C} \\
\mathrm{SC} \\
\mathrm{SC}\end{array}$ \\
\hline $\begin{array}{l}\text { EAFB }-5 \\
E B T-28 \\
E E T-29 \\
E B T-30 \\
E B T-31\end{array}$ & $\begin{array}{l}\text { Fortsmouth } \\
\text { East Boston } \\
\text { East Boston } \\
\text { East Boston } \\
\text { East Boston }\end{array}$ & & $\begin{array}{l}- \\
3 / 4 \\
3 / 4 \\
3 / 4 \\
3 / 4\end{array}$ & $\begin{array}{l}100 \\
86 \\
86 \\
86 \\
86\end{array}$ & $\begin{array}{l}100 \\
73 \\
73 \\
73 \\
73\end{array}$ & $\begin{array}{l}36 \\
57 \\
57 \\
57 \\
57\end{array}$ & $\begin{array}{l}49 \\
49 \\
49 \\
49 \\
49\end{array}$ & $\begin{array}{l}38 \\
42 \\
42 \\
42 \\
42\end{array}$ & $\begin{array}{l}30 \\
30 \\
30 \\
30 \\
30\end{array}$ & $\begin{array}{l}- \\
- \\
-\end{array}$ & $\begin{array}{l}- \\
- \\
- \\
- \\
-\end{array}$ & $\left|\begin{array}{l}30.0 \\
21.0 \\
21.0 \\
21.0 \\
21.0\end{array}\right|$ & \begin{tabular}{r|}
11.7 \\
7.0 \\
7.0 \\
7.0 \\
7.0 \\
\end{tabular} & $\begin{array}{l}2.73 \\
2.75 \\
2.75 \\
2.75 \\
2.76\end{array}$ & $\begin{array}{l}110.3(\mathrm{e}) \\
130.8(\mathrm{~d}) \\
130.8(\mathrm{~d}) \\
130.8(\mathrm{~d}) \\
130.8(\mathrm{~d})\end{array}$ & 17.7 & $\begin{array}{l}109 \\
129 \\
130 \\
129 \\
131\end{array}$ & $\begin{array}{r}99 \\
98 \\
99 \\
98 \\
100\end{array}$ & $\begin{array}{l}0.569 \\
0.336 \\
0.328 \\
0.336 \\
0.317\end{array}$ & \begin{tabular}{r|}
95 \\
100 \\
100 \\
100 \\
100
\end{tabular} & $\mid \begin{array}{c}0 \\
0.012 \\
0.011 \\
0.012 \\
0.009\end{array}$ & $\begin{array}{l}17.8 . \\
12.2 \\
11.9 \\
12.2 \\
11.5\end{array}$ & \begin{tabular}{l|l|}
60.7 & 1 \\
37.4 \\
29.9 \\
32.0 \\
18.3
\end{tabular} & \begin{tabular}{|r|} 
\\
$\mid 12.7$ \\
72.5 \\
48.3 \\
51.2 \\
22.8
\end{tabular} & $\begin{array}{l}4.5 \\
7.8 \\
7.3 \\
8.0 \\
4.5\end{array}$ & $\begin{array}{r}12.8 \\
12.7 \\
.2 \\
10.5 \\
5.7\end{array}$ & $\left|\begin{array}{l}2.84 \\
1.52 \\
1.25 \\
1.31 \\
1.24\end{array}\right|$ & 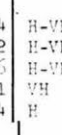 & $\begin{array}{l}\mathrm{SC} \\
\mathrm{SC} \\
\mathrm{SC} \\
\mathrm{SC} \\
\mathrm{SC}\end{array}$ \\
\hline $\begin{array}{l}\text { DFC-12 } \\
\text { DFC-13 } \\
\text { ART-2 } \\
\text { ART }-3 \\
\text { ART }-4\end{array}$ & $\begin{array}{l}\text { Dow Field } \\
\text { Dow Field } \\
\text { AASHO } \\
\text { AASHO } \\
\text { AASHO }\end{array}$ & & $\begin{array}{l}3 / 4 \\
3 / 4 \\
1 \frac{1}{3} \\
1 \frac{1}{2} \\
1 \frac{1}{2}\end{array}$ & $\begin{array}{l}96 \\
85 \\
95 \\
35 \\
35\end{array}$ & $\begin{array}{l}93 \\
82 \\
87 \\
87 \\
87\end{array}$ & $\begin{array}{l}86 \\
78 \\
74 \\
74 \\
74\end{array}$ & $\begin{array}{l}51 \\
53 \\
58 \\
58 \\
58\end{array}$ & $\begin{array}{l}38 \\
40 \\
48 \\
48 \\
48\end{array}$ & $\begin{array}{l}27 \\
30 \\
38 \\
38 \\
38\end{array}$ & $\begin{array}{l}- \\
- \\
- \\
-\end{array}$ & 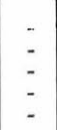 & \begin{tabular}{|l|}
26.4 \\
27.6 \\
27.3 \\
27.3 \\
27.3
\end{tabular} & $\left\{\begin{array}{r}8.4 \\
3.5 \\
11.9 \\
11.9 \\
11.9\end{array}\right.$ & $\begin{array}{l}2.67 \\
2.73 \\
2.74 \\
2.74 \\
2.74\end{array}$ & $\left|\begin{array}{l}112.3 \\
119.0 \\
121.0 \\
121.0 \\
121.0 \\
\text { (d) } \\
\text { (a) } \\
\text { (a) }\end{array}\right|$ & $\begin{array}{l}14.0 \\
14.0 \\
13.5 \\
13.5 \\
13.5\end{array}$ & $\begin{array}{l}118 \\
119 \\
117 \\
120 \\
125\end{array}$ & $\begin{array}{r}98 \\
99 \\
97 \\
99 \\
103\end{array}$ & $\begin{array}{l}0.424 \\
0.429 \\
0.467 \\
0.420 \\
0.367\end{array}$ & $\begin{array}{l}100 \\
100 \\
100 \\
100 \\
100\end{array}$ & \begin{tabular}{l|}
0.0028 \\
0.00007 \\
0.0047 \\
0.0020 \\
0.0008
\end{tabular} & $\begin{array}{l}15.7 \\
15.8 \\
17.2 \\
15.5 \\
13.5\end{array}$ & $\left|\begin{array}{l}79.1 \\
65.0 \\
25.4 \\
20.2 \\
18.6\end{array}\right|$ & \begin{tabular}{|r|}
67.0 \\
27.0 \\
28.4 \\
17.1 \\
13.9
\end{tabular} & $\begin{array}{l}6.2 \\
6.6 \\
2.3 \\
1.3 \\
1.1\end{array}$ & \begin{tabular}{r|}
10.7 \\
11.0 \\
4.3 \\
2.0 \\
2.3
\end{tabular} & $\begin{array}{l}1.72 \\
1.5 \\
1.85 \\
1.54 \\
2.07\end{array}$ & 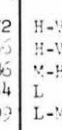 & $\begin{array}{l}1 \\
\text { S SC } \\
\text { SC } \\
3\end{array}$ \\
\hline $\begin{array}{l}\text { ART }-15 \\
\text { ART }-16 \\
\text { BOSG }-4 \\
\text { BOSG-6 } \\
\text { BOSG }-3\end{array}$ & $\begin{array}{l}\text { AASHO } \\
\text { AASHO } \\
\text { Bong } \\
\text { Bong } \\
\text { Bong }\end{array}$ & & $\begin{array}{l}1 \frac{1}{4} \\
1 \frac{1}{5} \\
3 / 4 \\
3 / 4 \\
3 / 4\end{array}$ & $\begin{array}{l}35 \\
35 \\
37 \\
97 \\
37\end{array}$ & $\begin{array}{l}87 \\
87 \\
70 \\
70 \\
91\end{array}$ & $\begin{array}{l}74 \\
74 \\
80 \\
80 \\
81\end{array}$ & $\begin{array}{l}58 \\
58 \\
50 \\
60 \\
51\end{array}$ & $\begin{array}{l}48 \\
48 \\
48 \\
48 \\
50\end{array}$ & $\begin{array}{l}38 \\
38 \\
36 \\
36 \\
35\end{array}$ & $\begin{array}{l}- \\
- \\
- \\
-\end{array}$ & $\begin{array}{l}- \\
- \\
-\end{array}$ & \begin{tabular}{|l|}
27.3 \\
27.3 \\
28.0 \\
28.6 \\
29.6
\end{tabular} & $\begin{array}{l}11.2 \\
11 . \\
12.5 \\
12.6 \\
13.6\end{array}$ & $\begin{array}{l}2.74 \\
2.74 \\
2.80 \\
2.80 \\
2.80\end{array}$ & 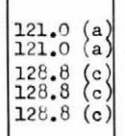 & $\begin{array}{l}13.5 \\
13.5\end{array}$ & $\begin{array}{l}119 \\
126 \\
125 \\
125 \\
126\end{array}$ & $\begin{array}{r}98 \\
104 \\
98 \\
98 \\
98\end{array}$ & \begin{tabular}{|l|}
0.442 \\
0.360 \\
0.395 \\
0.403 \\
0.389
\end{tabular} & \begin{tabular}{|r|}
100 \\
100 \\
96 \\
100 \\
97
\end{tabular} & \begin{tabular}{l|}
0.0030 \\
0.0007 \\
0.00002 \\
0.00003 \\
0.00001
\end{tabular} & $\begin{array}{l}16.3 \\
13.3 \\
13.5 \\
14.5 \\
13.6\end{array}$ & $\begin{array}{l}26.2 \\
18.0 \\
16.1 \\
17.7 \\
16.7\end{array}$ & $\begin{array}{l}27.0 \\
10.1 \\
16.2 \\
17.4 \\
16.7\end{array}$ & $\begin{array}{l}2.8 \\
1.2 \\
1.5 \\
1,2 \\
1.4\end{array}$ & \begin{tabular}{l|}
3.8 \\
1.3 \\
1.7 \\
1.7 \\
1.5
\end{tabular} & $\begin{array}{l}1.35 \\
1.08 \\
1.13 \\
1.42 \\
1.07\end{array}$ & $\begin{array}{lll} & & \text { Y } \\
8 & \mathrm{~L} \\
3 & \mathrm{~L} \\
2 & \mathrm{~L} \\
\mathrm{2} & \mathrm{L} \\
7 & \mathrm{~L}\end{array}$ & 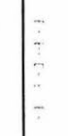 \\
\hline $\begin{array}{l}\text { DFT-1 } \\
\text { DFT-3 } \\
\end{array}$ & $\begin{array}{l}\text { Dow Field } \\
\text { Dow Field }\end{array}$ & & $\begin{array}{l}1 \frac{1}{2} \\
1 \frac{1}{2}\end{array}$ & $\begin{array}{l}94 \\
34 \\
\end{array}$ & $\begin{array}{l}88 \\
88 \\
\end{array}$ & $\begin{array}{l}80 \\
80 \\
\end{array}$ & $\begin{array}{l}64 \\
64 \\
\end{array}$ & $\begin{array}{l}52 \\
52 \\
\end{array}$ & $\begin{array}{l}37 \\
37 \\
\end{array}$ & $\therefore$ & - & \begin{tabular}{|l|}
30.0 \\
30.0
\end{tabular} & $\mid \begin{array}{l}12.0 \\
12.0\end{array}$ & \begin{tabular}{|l|}
2.71 \\
2.71 \\
\end{tabular} & $\left|\begin{array}{ll}119.8 & \text { (d) } \\
119.8 & \text { (d) }\end{array}\right|$ & $\begin{array}{l}14.0 \\
14.0\end{array}$ & $\begin{array}{l}117 \\
118\end{array}$ & $\begin{array}{l}98 \\
98\end{array}$ & $\begin{array}{l}0.448 \\
0.431 \\
\end{array}$ & $\begin{array}{l}100 \\
100 \\
\end{array}$ & \begin{tabular}{l|l}
0.0032 \\
0.0026
\end{tabular} & $\begin{array}{l}16.4 \\
16.1 \\
1\end{array}$ & $\begin{array}{l}69.7 \\
42.4\end{array}$ & $\begin{array}{r}124.3 \\
79.7 \\
\end{array}$ & $\begin{array}{l}10.1 \\
3.3 \\
\end{array}$ & $\begin{array}{r}-12.0 \\
3.8 \\
\end{array}$ & $\begin{array}{l}1.20 \\
1.25 \\
\end{array}$ & $\begin{array}{ll}0 \\
0 \\
5\end{array}$ & $\begin{array}{l}\mathrm{SC} \\
\mathrm{SC}\end{array}$ \\
\hline
\end{tabular}


Table AI (Cont'd). Summary of frost susceptibility tests on natural soils ${ }^{(1)}$ - open-system nominal load pressure 0.5 psi.

\begin{tabular}{|c|c|c|c|c|c|c|c|c|c|c|c|c|c|c|c|c|c|c|c|c|c|c|c|c|c|c|c|c|c|}
\hline \multirow{3}{*}{$\begin{array}{l}\text { Specimen } \\
\text { Number }\end{array}$} & \multirow{3}{*}{ Material Source } & \multicolumn{10}{|c|}{ SOIL GRADATION DATA (AB Frozen) } & \multicolumn{5}{|c|}{ PHYSICAL PROPERTIES OF BASIC SOIL } & \multicolumn{5}{|c|}{ SPECIMEN DATA (As Molded) } & \multicolumn{7}{|c|}{ FREEZING TEST DATA } & \multirow{3}{*}{$\begin{array}{l}\text { Typo } \\
\text { of } \\
\text { cyt } \\
\text { (12) }\end{array}$} \\
\hline & & \multirow{2}{*}{$\begin{array}{l}\text { Unified } \\
\text { Soii } \\
\text { Clossifi- } \\
\text { cotion } \\
\text { Symbol } \\
\text { (2) }\end{array}$} & \multirow{2}{*}{$\begin{array}{c}\text { Moxi- } \\
\text { mum } \\
\text { Size } \\
\text { in. }\end{array}$} & \multicolumn{6}{|c|}{ Percent finer, mm } & \multicolumn{2}{|c|}{$\begin{array}{c}\text { Coof ficionts } \\
\text { (3) }\end{array}$} & \multicolumn{2}{|c|}{\begin{tabular}{|l|} 
Atterberg \\
Limits (4) \\
\end{tabular}} & \multirow{2}{*}{$\begin{array}{l}\text { Specific } \\
\text { Grovity }\end{array}$} & \multirow{2}{*}{\multicolumn{2}{|c|}{ 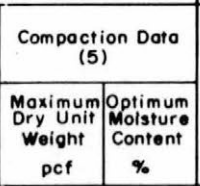 }} & \multirow{2}{*}{$\begin{array}{c}\text { Ory } \\
\text { Unit } \\
\text { Woight } \\
\text { pef } \\
\end{array}$} & \multirow{2}{*}{\begin{tabular}{|c} 
Dogree \\
of \\
Compoc- \\
tion \\
$x$ \\
\end{tabular}} & \multirow{2}{*}{\begin{tabular}{|c|} 
Void \\
Rotio \\
$\%$ \\
\end{tabular}} & \multirow{2}{*}{\begin{tabular}{|c|} 
G, of \\
Stort \\
of \\
Tost \\
$(6)$ \\
\% \\
\end{tabular}} & \multirow{2}{*}{$\begin{array}{c}\text { Porme- } \\
\text { obilify } \\
k \\
(7) \\
\mathrm{cm} / \mathrm{sec} \\
\times 10^{-4}\end{array}$} & \multicolumn{2}{|c|}{$\begin{array}{c}\text { Avg. } \\
\text { wottor } \\
\text { content }\end{array}$} & \multirow{2}{*}{\begin{tabular}{|c|} 
Totol \\
Toove \\
(8) \\
\% \\
\end{tabular}} & \multicolumn{2}{|c|}{\begin{tabular}{|c|}
$\begin{array}{c}\text { Rate of } \\
\text { Heave } \\
\text { mm / day (9) }\end{array}$ \\
\end{tabular}} & \multirow{2}{*}{$\begin{array}{l}\text { Heave } \\
\text { Rote } \\
\text { Vor. } \\
\text { Index } \\
\text { (10) } \\
\text { C }\end{array}$} & \multirow{2}{*}{$\begin{array}{l}\text { Frost } \\
\text { Susc } \\
\text { Cilos: } \\
\text { (III) }\end{array}$} & \\
\hline & & & & 4.76 & $0.42 \mid$ & 0.074 & 0.02 & 0.01 & 0.005 & $C_{u}$ & $\mathrm{c}_{\mathrm{c}}$ & LL & PI & & & & & & & & & $\begin{array}{c}\text { Before } \\
\text { To st } \\
x\end{array}$ & $\begin{array}{c}\text { After } \\
\text { Tost } \\
\%\end{array}$ & & Avo. & Max. ${ }^{I}$ & & & \\
\hline $\begin{array}{l}\text { PAFB-1A } \\
\text { CCL-1 } \\
\text { PBN-1 } \\
\text { YS - -7 }\end{array}$ & $\begin{array}{l}\text { Portsmouth } \\
\text { Crosby } \\
\text { Greenland } \\
\text { Yukon }\end{array}$ & CL & $=$ & $\begin{array}{l}100 \\
100 \\
100 \\
100\end{array}$ & $\begin{array}{l}98 \\
98 \\
100 \\
100\end{array}$ & $\begin{array}{r}91 \\
91 \\
97 \\
100\end{array}$ & $\begin{array}{l}33 \\
58 \\
60 \\
67\end{array}$ & $\begin{array}{l}24 \\
41 \\
43 \\
37\end{array}$ & $\begin{array}{l}19 \\
31 \\
34 \\
29\end{array}$ & $\overline{-}$ & 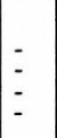 & \begin{tabular}{|l|l}
28.0 & 1 \\
36.5 & 1 \\
31.3 & 1 \\
28.0 &
\end{tabular} & $\begin{array}{l}\text { LEA } \\
12.0 \\
16.8 \\
15.2 \\
8.6\end{array}$ & \begin{tabular}{|l|}
2.71 \\
2.78 \\
2.79 \\
2.74
\end{tabular} & $\left|\begin{array}{ll}113.4 & (d) \\
119.3 & (c) \\
119.4 & (d) \\
121.4 & (d)\end{array}\right|$ & $\begin{array}{l}13 . \overline{5} \\
15.0 \\
12.8\end{array}$ & $\begin{array}{l}113 \\
117 \\
116 \\
117\end{array}$ & $\begin{array}{r}100 \\
98 \\
37 \\
96\end{array}$ & $\mid \begin{array}{l}0.474 \\
0.485 \\
0.518 \\
0.460\end{array}$ & $\begin{array}{r}92 \\
100 \\
100 \\
89\end{array}$ & $\begin{array}{c}- \\
- \\
0.000003\end{array}$ & $\begin{array}{l}16.3 \\
17.5 \\
18.3 \\
15.0\end{array}$ & \begin{tabular}{|l|}
38.0 \\
24.6 \\
30.1 \\
22.0
\end{tabular} & $\begin{array}{l}47.1 \\
17.7 \\
26.8 \\
24.0\end{array}$ & $\begin{array}{l}4.0 \\
1.4 \\
2.2 \\
1.1\end{array}$ & $\begin{array}{l}4.8 \\
2.3 \\
5.3 \\
2.5\end{array}$ & $\begin{array}{l}1.20 \\
1.64 \\
2.40 \\
2.27\end{array}$ & \begin{tabular}{l|}
$H$ \\
$L-M$ \\
$M-H$ \\
$L-M$
\end{tabular} & \begin{tabular}{|l} 
SL \\
T \\
$T$ \\
SL
\end{tabular} \\
\hline \begin{tabular}{l|} 
YS-8 \\
YS-14 \\
YS-15 \\
YS-16 \\
\end{tabular} & \begin{tabular}{|l} 
Yukon \\
Yukon \\
Yukon \\
Yukon \\
\end{tabular} & & $\begin{array}{l}- \\
- \\
- \\
\end{array}$ & $\begin{array}{l}100 \\
100 \\
100 \\
100 \\
\end{array}$ & $\begin{array}{l}100 \\
100 \\
100 \\
100 \\
\end{array}$ & $\begin{array}{l}100 \\
100 \\
100 \\
100 \\
\end{array}$ & $\begin{array}{l}67 \\
67 \\
67 \\
67 \\
\end{array}$ & $\begin{array}{l}37 \\
37 \\
37 \\
37 \\
\end{array}$ & $\begin{array}{l}29 \\
29 \\
29 \\
29 \\
\end{array}$ & 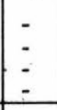 & 5 & \begin{tabular}{|l|}
28.0 \\
28.0 \\
28.0 \\
23.0 \\
\end{tabular} & \begin{tabular}{l|}
8.6 \\
8.6 \\
8.6 \\
8.6 \\
\end{tabular} & \begin{tabular}{|l}
2.74 \\
2.74 \\
2.74 \\
2.74 \\
\end{tabular} & $\begin{array}{l}121.4 \text { (d) } \\
121.4 \\
121.4 \\
121.4 \\
\text { (d) } \\
\text { (d) }\end{array}$ & $\begin{array}{l}12.8 \\
12.8 \\
12.8 \\
12.8 \\
\end{array}$ & $\begin{array}{l}118 \\
123 \\
120 \\
115 \\
\end{array}$ & $\begin{array}{r}97 \\
101 \\
98 \\
95 \\
\end{array}$ & $\begin{array}{l}0.448 \\
0.385 \\
0.424 \\
0.476 \\
\end{array}$ & $\begin{array}{r}94 \\
100 \\
100 \\
94 \\
\end{array}$ & $\begin{array}{l}0.000002 \\
0.0000006 \\
0.000001 \\
0.000005\end{array}$ & \begin{tabular}{|l|}
15.4 \\
614.1 \\
15.5 \\
16.5 \\
\end{tabular} & \begin{tabular}{|l|}
33.0 \\
29.5 \\
29.1 \\
36.6 \\
\end{tabular} & \begin{tabular}{|l|}
45.7 \\
38.5 \\
34.3 \\
46.2 \\
\end{tabular} & \begin{tabular}{|l|}
3.8 \\
2.1 \\
1.8 \\
2.5 \\
\end{tabular} & \begin{tabular}{l|}
5.3 \\
4.0 \\
3.7 \\
4.2 \\
\end{tabular} & \begin{tabular}{|l|}
1.33 \\
1.90 \\
2.06 \\
1.68 \\
\end{tabular} & $\begin{array}{l}M-H \\
M \\
L-M \\
M-H\end{array}$ & $\begin{array}{l}\text { SC } \\
\text { SC } \\
\text { SC } \\
\text { SC } \\
\end{array}$ \\
\hline $\begin{array}{l}\text { WASHO-1 } \\
\text { WASHO-5 } \\
\text { WASHO-6 } \\
\text { WASHO-7 }\end{array}$ & $\begin{array}{l}\text { Malad, Idaho } \\
\text { Malad, Idaho } \\
\text { Malad, Idaho } \\
\text { Malad, Idaho } \\
\end{array}$ & CL-OL & $\begin{array}{l}= \\
=\end{array}$ & $\begin{array}{l}100 \\
100 \\
100 \\
100 \\
\end{array}$ & $\begin{array}{l}99 \\
99 \\
99 \\
99 \\
\end{array}$ & $\begin{array}{l}96 \\
96 \\
96 \\
96 \\
\end{array}$ & $\begin{array}{l}65 \\
65 \\
65 \\
65 \\
\end{array}$ & $\begin{array}{l}48 \\
48 \\
48 \\
48 \\
\end{array}$ & $\begin{array}{l}35 \\
35 \\
35 \\
35 \\
\end{array}$ & $=$ & $\bar{z}$ & $\begin{array}{l}\text { LEAN } \\
37.00 \\
377.0 \\
37.01 \\
37.0 \\
\end{array}$ & $\begin{array}{l}\text { CLAYS } \\
23.0 \\
23.0 \\
23.0 \\
23.0 \\
23.0\end{array}$ & \begin{tabular}{|l}
2.58 \\
2.58 \\
2.58 \\
2.58 \\
2.58 \\
\end{tabular} & $\begin{array}{r}\text { ANICS } \\
99.6 \\
99.6 \\
99.6 \\
99.6 \\
\end{array}$ & \begin{tabular}{|l|l|}
21.0 & $(c)$ \\
21.0 & $(c)$ \\
21.0 & $(c)$ \\
21.0 & $c$ \\
\end{tabular} & $\begin{array}{l}99 \\
96 \\
98 \\
99 \\
\end{array}$ & $\begin{array}{l}99 \\
96 \\
98 \\
99 \\
\end{array}$ & \begin{tabular}{|l|}
0.630 \\
0.678 \\
0.644 \\
0.627 \\
0.627 \\
\end{tabular} & $\begin{array}{l}100 \\
100 \\
100 \\
100 \\
\end{array}$ & $\overline{-}$ & $\begin{array}{l}24.4 \\
26.3 \\
25.0 . \\
24.3 \\
\end{array}$ & $\left|\begin{array}{l}31.4 \\
60.8 \\
42.5 \\
45.0\end{array}\right|$ & $\begin{array}{l}20.9 \\
61.0 \\
42.3 \\
45.0 \\
\end{array}$ & $\begin{array}{l}3.4 \\
4.6 \\
4.1 \\
4.2 \\
\end{array}$ & $\begin{array}{l}4.0 \\
7.3 \\
5.2 \\
5.0 \\
\end{array}$ & \begin{tabular}{|l|l}
1.18 & \\
1.58 & \\
1.26 & 1 \\
1.19 & \\
\end{tabular} & \begin{tabular}{|l}
$\mathrm{M}$ \\
$\mathrm{H}$ \\
$\mathrm{H}$ \\
$\mathrm{H}$ \\
$\mathrm{H}$ \\
\end{tabular} & $\begin{array}{l}\mathrm{sc} \\
\mathrm{SC} \\
\mathrm{SC} \\
\mathrm{SC} \\
\end{array}$ \\
\hline FCH-1 & Frederick & $\mathrm{CH}$ & - & 200 & 99 & 74 & 61 & 52 & 43 & - & - & 55.03 & & $\begin{array}{l}\frac{T \text { CLAYS }}{2.88} \\
\end{array}$ & 106.7 & 19.5 (c) & 105 & 98 & 0.715 & 86 & - & 21.2 & 38.4 & 39.0 & 0.8 & 1.7 & 2.12 & VL-L 7 & \\
\hline
\end{tabular}


Table AII. Summary of frost susceptibility tests on natural solls ${ }^{(1)}$ - open-system nominal load pressure 0.5 psi.

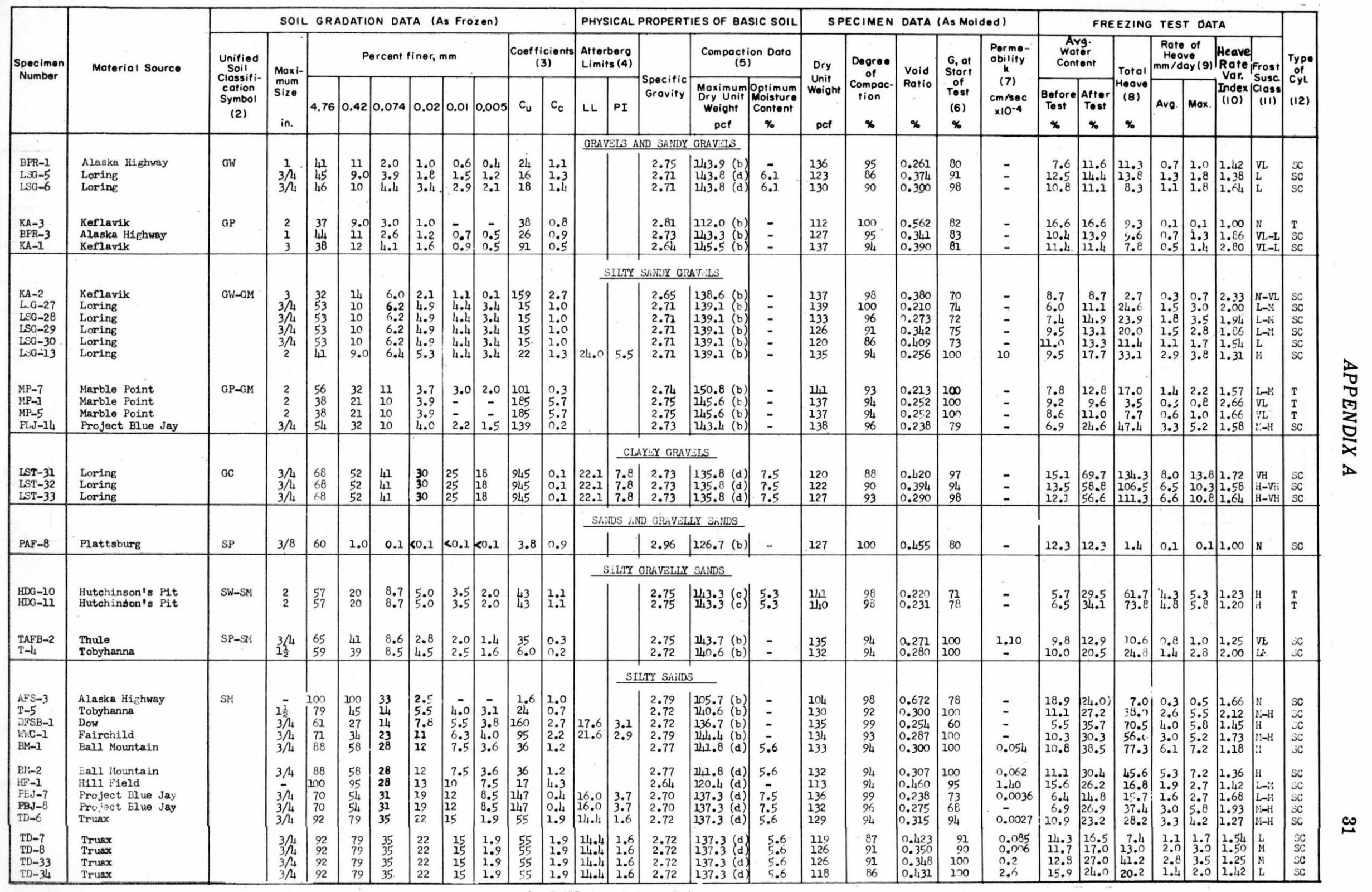


Table AII (Cont'd). Summary of frost susceptibility tests on natural soils ${ }^{(1)}$ - open-system nominal load pressure 0.5 psi.

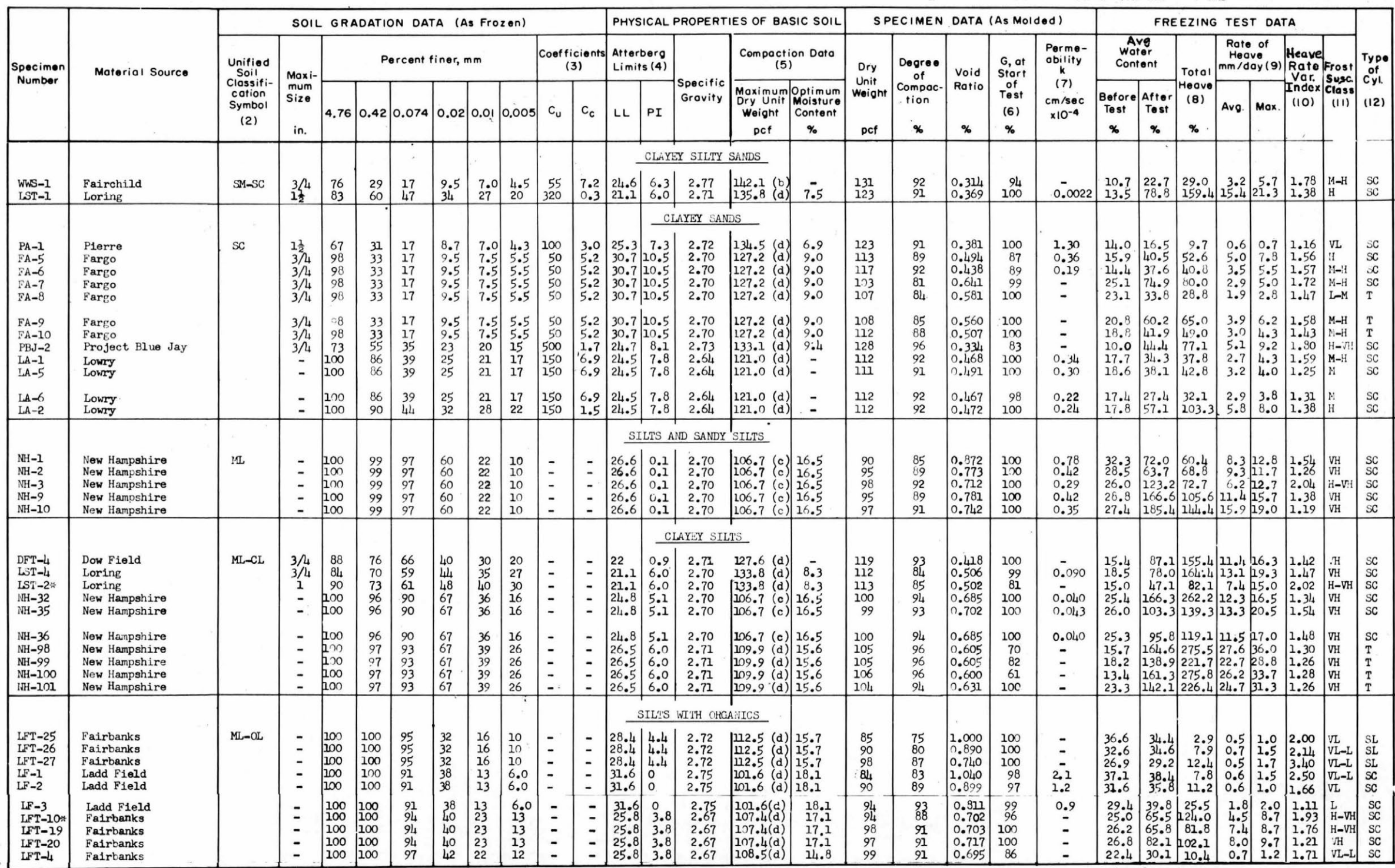


Table AII (Cont'd).

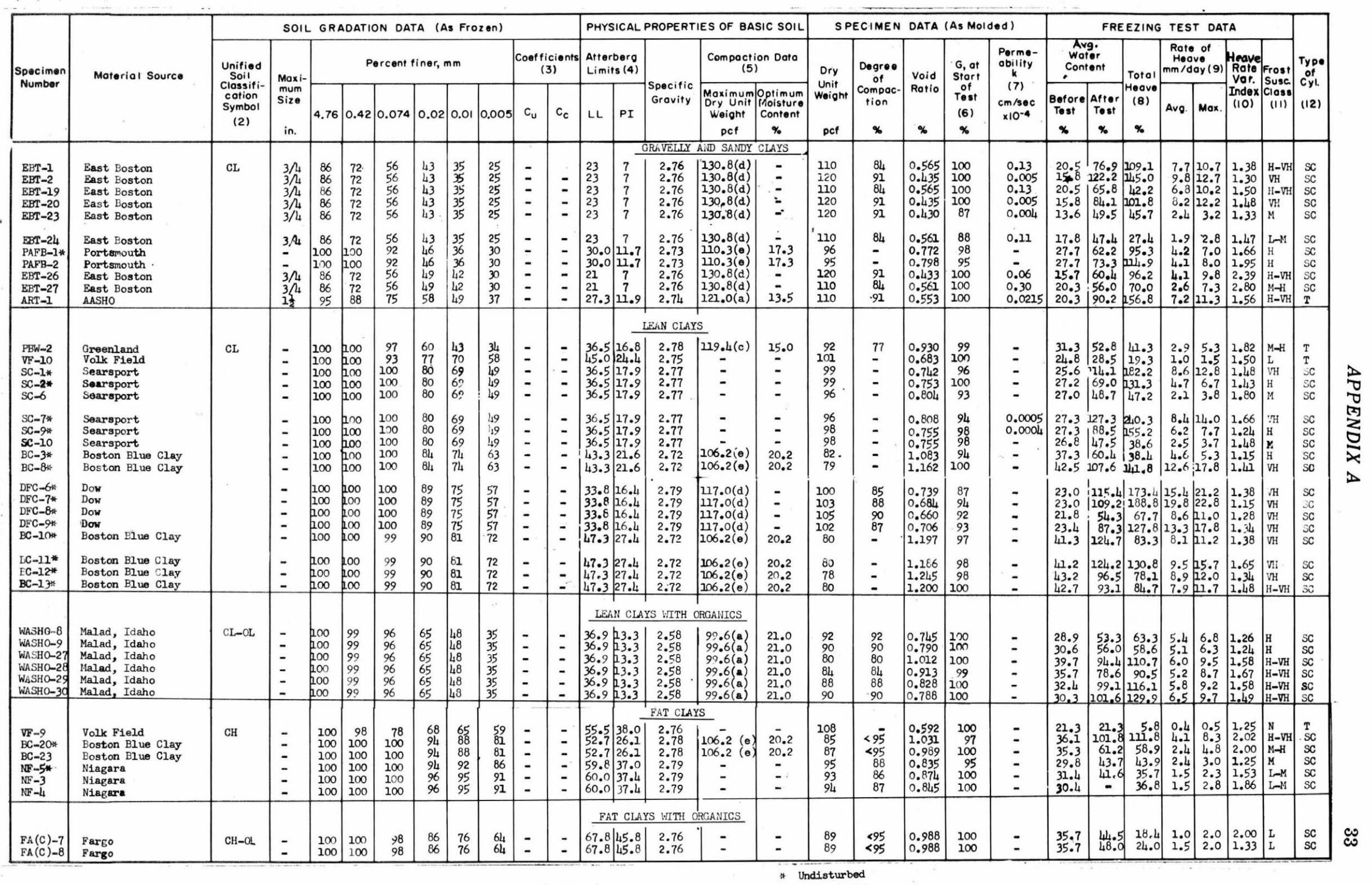


Table AIII. Summary of frost susceptibility tests on natural soils ${ }^{(14)}$ - open-system nominal load pressure 0.073 psi.

\begin{tabular}{|c|c|c|c|c|c|c|c|c|c|c|c|c|c|c|c|c|c|c|c|c|c|c|c|c|c|c|c|c|c|}
\hline \multirow{3}{*}{$\begin{array}{c}\text { Specimon } \\
\text { Number }\end{array}$} & \multirow{3}{*}{ Moterial Source } & \multicolumn{10}{|c|}{ SOIL GRADATION DATA (As Frozen) } & \multicolumn{5}{|c|}{ PHYSICAL PROPERTIES OF BASIC SOIL } & \multicolumn{5}{|c|}{ SPECIMEN DATA (As Molded) } & \multicolumn{7}{|c|}{ FREEZING TEST DATA } & \multirow{3}{*}{$\begin{array}{l}\text { Typ } \\
\text { of } \\
\text { Cyt. } \\
\text { (12) }\end{array}$} \\
\hline & & \multirow{2}{*}{$\begin{array}{l}\text { Unified } \\
\text { Soil1 } \\
\text { clossifi- } \\
\text { cotion } \\
\text { Symbol } \\
\text { (2) }\end{array}$} & \multirow{2}{*}{\begin{tabular}{|c|} 
Moxi- \\
mum \\
Size \\
in. \\
\end{tabular}} & \multicolumn{6}{|c|}{ Percent finer, $\mathrm{mm}$} & \multicolumn{2}{|c|}{$\begin{array}{c}\text { Coofficients } \\
\text { (3) }\end{array}$} & \multicolumn{2}{|c|}{$\begin{array}{l}\text { Atterberg } \\
\text { Limits (4) }\end{array}$} & \multirow{2}{*}{$\begin{array}{l}\text { Specific } \\
\text { Grovity }\end{array}$} & \multicolumn{2}{|c|}{$\begin{array}{c}\text { Compaction Dato } \\
\text { (5) }\end{array}$} & \multirow{2}{*}{\begin{tabular}{|c} 
Dry \\
Unit \\
Woight \\
pef
\end{tabular}} & \multirow{2}{*}{$\begin{array}{c}\text { Degree } \\
\text { of } \\
\text { Compac- } \\
\text { tion } \\
x\end{array}$} & \multirow{2}{*}{\begin{tabular}{|c|} 
Void \\
Ratio \\
$\%$ \\
\end{tabular}} & \multirow{2}{*}{\begin{tabular}{|c|} 
G, ot \\
Stort \\
of \\
Test \\
$(6)$ \\
$\%$ \\
\end{tabular}} & \multirow{2}{*}{$\begin{array}{c}\text { Perme- } \\
\text { abiitify } \\
k \\
(7) \\
\mathrm{cm} / \mathrm{sec} \\
\times 10^{-4}\end{array}$} & \multicolumn{2}{|c|}{$\begin{array}{l}\text { Avg } \\
\text { Wotor } \\
\text { Content }\end{array}$} & \multirow{2}{*}{$\begin{array}{c}\text { Totol } \\
\text { Heove } \\
(8) \\
\%\end{array}$} & \multicolumn{2}{|c|}{\begin{tabular}{|l|}
$\begin{array}{l}\text { Rate of of } \\
\text { Heove } \\
\text { mm/doy (9) }\end{array}$ \\
\end{tabular}} & \multirow{2}{*}{$\begin{array}{l}\text { Heave } \\
\text { Hate } \\
\text { Var. } \\
\text { Vardex } \\
\text { (10) }\end{array}$} & \multirow{2}{*}{$\begin{array}{l}\text { Fest } \\
\text { Fusc } \\
\text { Sulos: } \\
\text { CliI) }\end{array}$} & \\
\hline & & & & 4.76 & 0.42 & 0.074 & 0.02 & 0.01 & 0.005 & $c_{u}$ & $c_{c}$ & LL & PI & & {$\left[\begin{array}{c}\text { Dry Unif } \\
\text { Weight } \\
\text { pcf }\end{array} \mid\right.$} & $\begin{array}{c}\text { Moisture } \\
\text { Content } \\
\%\end{array}$ & & & & & & \begin{tabular}{|c} 
Before \\
Test \\
$\%$
\end{tabular} & \begin{tabular}{|c|} 
After \\
Tost \\
$\%$
\end{tabular} & & Avg. & Max. & & & \\
\hline $\begin{array}{l}\text { GRAVELS } \\
\text { ADG-1 } \\
\end{array}$ & $\begin{array}{l}\text { and SANDY GRAVELS } \\
\text { Alaska Highway }\end{array}$ & GW & 2 & 40 & 10 & 3.7 & 1.9 & 91.5 & 5.9 & 22 & 1.6 & & & 2.64 & $233.4(\mathrm{~b})$ & - & 132 & 99 & 0.249 & 100 & - & 9.4 & 11.6 & 1.9 & 0.9 & 1.3 & 1.45 & VILI & $\mathrm{sc}$ \\
\hline $\begin{array}{l}\text { SILTY SA } \\
\text { AMS-1 } \\
\text { AMS-2 } \\
\text { ACR-1 } \\
\text { ACR-2 }\end{array}$ & $\begin{array}{l}\text { NDY GRAVELS } \\
\text { Alaska Highway } \\
\text { Alaska Highway } \\
\text { Alaska Highway } \\
\text { Alaska Highway }\end{array}$ & $\begin{array}{l}G P-G M \\
G W-G M \\
G P-G M \\
G P-G M\end{array}$ & $\begin{array}{l}2 \\
2 \\
2 \\
2 \\
\end{array}$ & $\begin{array}{l}27 \\
44 \\
34 \\
37\end{array}$ & $\begin{array}{l}10 \\
16 \\
18 \\
20 \\
\end{array}$ & $\begin{array}{l}5.2 \\
7.2 \\
11 \\
12\end{array}$ & $\begin{array}{l}3.1 \\
5.4 \\
6.2 \\
8.5\end{array}$ & $\begin{array}{ll}1 & 2.0 \\
& 3.8 \\
2 & 4.2 \\
5 & 6.5 \\
\end{array}$ & \begin{tabular}{|l|}
1.2 \\
8.4 \\
2.7 \\
5.1 \\
5
\end{tabular} & $\begin{array}{r}40 \\
67 \\
440 \\
310 \\
\end{array}$ & $\begin{array}{l}4.7 \\
2.2 \\
6.2 \\
3.1 \\
\end{array}$ & $\begin{array}{l}38.6 \\
38.6 \\
25.7 \\
25.7\end{array}$ & $\begin{array}{l}2.7 \\
2.7 \\
3.6 \\
3.6 \\
\end{array}$ & $\begin{array}{l}2.73 \\
2.73 \\
2.72 \\
2.70 \\
\end{array}$ & $\begin{array}{l}123.6 \text { (b) } \\
118.5 \text { (b) } \\
127.0 \text { (b) } \\
226.7 \text { (b) }\end{array}$ & $\begin{array}{l}5 \\
\vdots\end{array}$ & $\begin{array}{l}121 \\
121 \\
126 \\
128 \\
\end{array}$ & $\begin{array}{r}98 \\
102 \\
99 \\
101\end{array}$ & $\begin{array}{l}0.401 \\
0.401 \\
0.336 \\
0.315\end{array}$ & $\begin{array}{r}100 \\
100 \\
77 \\
94 \\
\end{array}$ & $\begin{array}{l}\overline{-} \\
\overline{-}\end{array}$ & \begin{tabular}{|l|}
14.7 \\
10.6 \\
9.5 \\
11.0 \\
\end{tabular} & $\begin{array}{l}18.3 \\
20.8 \\
20.8 \\
19.6\end{array}$ & $\begin{array}{l}17.6 \\
17.6 \\
30.5 \\
29.7\end{array}$ & \begin{tabular}{l|}
1.1 \\
2.4 \\
1.9 \\
1.9
\end{tabular} & \begin{tabular}{l|}
- \\
2.5 \\
3.8 \\
3.7 \\
3.3
\end{tabular} & $\begin{array}{l}2.27 \\
1.65 \\
1.95 \\
1.74\end{array}$ & $\begin{array}{l}L-M \\
M-M \\
L-M \\
L-M\end{array}$ & $\begin{array}{l}\text { SC } \\
\text { SC } \\
\text { SC } \\
\text { SC }\end{array}$ \\
\hline$\frac{\text { SILTY GI }}{\text { BM-6 }}$ & $\frac{\text { AVELS }}{\text { Bal! I. Nountain Till }}$ & GI & 2 & 91 & 35 & 18 & 7 & - & - & 250 & 0.3 & & & 2.81 & - & - & 147 & - & 0.195 & 100 & - & 6.6 & 11.7 & 17.4 & 1.4 & 3.8 & 2.71 & L-M & $\mathrm{T}$ \\
\hline $\begin{array}{l}\text { SANDS ar } \\
\text { ADG-2 } \\
\end{array}$ & $\frac{\text { a GRAVBLLLY SANDS }}{\text { hlaska Highway }}$ & SW & 2 & 53 & 13 & 3.8 & 1.8 & 81.4 & 0.9 & 20 & 1.1 & & & 2.65 & 132.9 (b) & - & 129 & 97 & 0.277 & 100 & - & 10.5 & 12.2 & 10.2 & 1.0 & 1.7 & 1.70 & L & sc \\
\hline $\begin{array}{l}\text { SILTY SE } \\
\text { AiFS-1 } \\
\text { AFS-2 }\end{array}$ & $\begin{array}{l}\frac{\text { NDS }}{\text { Mlaska Highway }} \\
\text { Alaska Highway }\end{array}$ & $\operatorname{SM}$ & $=$ & $\begin{array}{l}100 \\
100\end{array}$ & $\begin{array}{l}100 \\
100\end{array}$ & $\begin{array}{l}33 \\
33\end{array}$ & $\begin{array}{l}2.5 \\
2.5\end{array}$ & $\overline{-}$ & $\overline{-}$ & $\begin{array}{l}1.6 \\
1.6\end{array}$ & $\begin{array}{l}1.0 \\
1.0\end{array}$ & & & $\begin{array}{l}2.79 \\
2.79\end{array}$ & $\begin{array}{l}276.4 \text { (b) } \\
206.4 \text { (b) }\end{array}$ & $\overline{-}$ & $\begin{array}{l}112 \\
111\end{array}$ & $\begin{array}{l}105 \\
105\end{array}$ & $\begin{array}{l}0.551 \\
0.565\end{array}$ & $\begin{array}{r}92 \\
100\end{array}$ & $=$ & $\begin{array}{l}18.2 \\
20.3\end{array}$ & $\begin{array}{r}32.8 \\
29.3\end{array}$ & $\begin{array}{l}20.0 \\
11.1\end{array}$ & $\begin{array}{l}2.0 \\
1.1\end{array}$ & $\begin{array}{l}3.0 \\
1.7\end{array}$ & $\begin{array}{l}1.50 \\
1.54\end{array}$ & M & $\begin{array}{l}\text { sc } \\
\text { sC }\end{array}$ \\
\hline$\frac{\text { CLAYEY S }}{\text { LST-6 }}$ & $\frac{\text { ILTY SANDS }}{\text { Limestone Till }}$ & SM-SC & $3 / 4$ & 84 & 65 & 49.7 & 36 & 30 & 21 & 225 & 1.0 & 21.1 & 6.0 & 2.72 & 233.8 (d) & 8.3 & 133 & 99 & 0.279 & 100 & - & 10.2 & 17.1 & 24.7 & 1.4 & 2.7 & 1.93 & $L-M$ & sc \\
\hline $\begin{array}{l}\text { SILTS ar } \\
\text { VIS-3 } \\
\text { VIS-7 } \\
\text { NH-13 } \\
\end{array}$ & 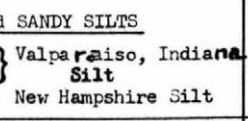 & if & $\overline{-}$ & \begin{tabular}{|l|}
100 \\
100 \\
100 \\
\end{tabular} & \begin{tabular}{|r|}
100 \\
100 \\
79 \\
\end{tabular} & $\begin{array}{l}99 \\
99 \\
97 \\
\end{array}$ & $\begin{array}{l}54 \\
54 \\
60\end{array}$ & $\begin{array}{l}25 \\
25 \\
22 \\
\end{array}$ & $\begin{array}{l}15 \\
15 \\
10 \\
\end{array}$ & $\overline{-}$ & $=$ & $\begin{array}{l}23.7 \\
23.7 \\
26.6 \\
\end{array}$ & \begin{tabular}{|l|}
4.0 \\
4.0 \\
0.1 \\
\end{tabular} & $\begin{array}{l}2.72 \\
2.72 \\
2.70\end{array}$ & $\begin{array}{ll}215.8 & (d) \\
115.8 & (d) \\
106.7 & (c) \\
1\end{array}$ & $\begin{array}{l}13.5 \\
13.5 \\
16.5\end{array}$ & $\begin{array}{l}112 \\
112 \\
105\end{array}$ & $\begin{array}{l}96 \\
96 \\
99\end{array}$ & $\overline{-}-$ & $\begin{array}{r}72 \\
94 \\
100\end{array}$ & $\begin{array}{l}.025 \\
.026 \\
0.15\end{array}$ & $\begin{array}{l}13.5 \\
17.7 \\
22.5 \\
\end{array}$ & $\begin{array}{r}53.1 \\
45.2 \\
105.81 \\
\end{array}$ & $\begin{array}{l}81.4 \\
442.3 \\
155.1\end{array}$ & \begin{tabular}{|r|r|}
6.8 & 1 \\
5.8 & 1 \\
11.7 & 1 \\
\end{tabular} & $\begin{array}{l}11.0 \\
11.5 \\
17.8\end{array}$ & $\begin{array}{l}1.62 \\
1.98 \\
1.52\end{array}$ & $\begin{array}{l}\mathrm{H}-\mathrm{VH} \\
\mathrm{H}-\mathrm{VH} \\
\mathrm{VH}\end{array}$ & \begin{tabular}{|l} 
SC \\
SC \\
SC
\end{tabular} \\
\hline $\begin{array}{l}\text { SILTS W } \\
\text { LF-10 } \\
\text { LFT }-9\end{array}$ & $\begin{array}{l}\text { ORGANICS } \\
\text { Ladd Field Silt } \\
\text { Fairbanks Silt }\end{array}$ & II $=0 \mathrm{~L}$ & $\overline{-}$ & $\begin{array}{l}100 \\
100\end{array}$ & $\begin{array}{l}100 \\
100\end{array}$ & $\begin{array}{l}91 \\
97\end{array}$ & $\begin{array}{l}38 \\
42\end{array}$ & $\begin{array}{l}13 \\
22\end{array}$ & $\begin{array}{l}6.0 \\
12\end{array}$ & $\overline{-}$ & $=$ & $\begin{array}{l}31.6 \\
32.6\end{array}$ & $\begin{array}{l}0.2 \\
6.2 \\
\end{array}$ & $\begin{array}{l}2.75 \\
2.67\end{array}$ & $\begin{array}{lll}101.6 & (d) \\
107.4 & \left(\begin{array}{l}c \\
c\end{array}\right) & 1\end{array}$ & $\begin{array}{l}18.1 \\
17.1\end{array}$ & $\begin{array}{r}99 \\
102\end{array}$ & $\begin{array}{l}92 \\
95\end{array}$ & $\begin{array}{l}0.724 \\
0.602\end{array}$ & $\begin{array}{l}100 \\
100\end{array}$ & 0.61 & \begin{tabular}{|l|}
26.4 \\
24.8
\end{tabular} & $\begin{array}{l}68.1 \\
61.0\end{array}$ & $\begin{array}{l}93.2 . \\
55.7\end{array}$ & $\begin{array}{l}7.1 \\
5.5\end{array} \mid$ & $\begin{array}{r}9.5 \\
11.3\end{array}$ & $\begin{array}{l}1.34 \\
2.05\end{array}$ & $\begin{array}{l}\mathrm{H}-\mathrm{VH} \\
\mathrm{H}-\mathrm{VH}\end{array}$ & $\begin{array}{l}s c \\
s c\end{array}$ \\
\hline $\begin{array}{l}\text { GRAVELL } \\
\text { EIT-13 } \\
\text { ART-7 } \\
\text { ART-13 } \\
\text { ART-19 } \\
\text { YS-3 } \\
\text { YS-10 }\end{array}$ & $\begin{array}{l}\text { and SANDY CLAYS } \\
\text { East Eoston Till } \\
\text { AASHO Road Test } \\
\text { AASHO Road Test } \\
\text { MASHO Road Test } \\
\text { Yukon Silt } \\
\text { Yukon Silt }\end{array}$ & CL & $\begin{array}{l}3 / 4 \\
1 \frac{1}{2} \\
1 \frac{1}{2} \\
1 \frac{2}{2} \\
- \\
-\end{array}$ & $\begin{array}{r}84 \\
95 \\
95 \\
95 \\
100 \\
100 \\
\end{array}$ & \begin{tabular}{|r|}
72 \\
87 \\
87 \\
87 \\
100 \\
100 \\
\end{tabular} & $\begin{array}{r}56 \\
74 \\
74 \\
74 \\
7100 \\
100 \\
\end{array}$ & $\begin{array}{l}43 \\
58 \\
58 \\
58 \\
67 \\
67 \\
\end{array}$ & $\begin{array}{l}35 \\
48 \\
48 \\
48 \\
37 \\
37 \\
\end{array}$ & $\begin{array}{l}25 \\
38 \\
38 \\
38 \\
29 \\
29 \\
\end{array}$ & $\begin{array}{l}z \\
z \\
z \\
-\end{array}$ & $\begin{array}{l}= \\
= \\
z \\
-\end{array}$ & $\begin{array}{l}23.0 \\
27.3 \\
27.3 \\
27.3 \\
28.0 \\
23.0 \\
\end{array}$ & $\begin{array}{r}7.0 \\
11.9 \\
11.9 \\
11.9 \\
8.6 \\
8.6 \\
\end{array}$ & $\begin{array}{l}2.76 \\
2.74 \\
2.74 \\
2.74 \\
2.74 \\
2.74 \\
\end{array}$ & 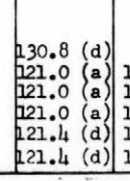 & $\begin{array}{l}13.5 \\
13.5 \\
13.5 \\
12.8 \\
12.8 \\
\end{array}$ & $\begin{array}{l}125 \\
116 \\
114 \\
122 \\
120 \\
118 \\
\end{array}$ & $\begin{array}{r}96 \\
96 \\
94 \\
105 \\
99 \\
97 \\
\end{array}$ & $\begin{array}{l}0.380 \\
0.481 \\
0.497 \\
0.4914 \\
0.443 \\
0.775 \\
\end{array}$ & $\begin{array}{r}100 \\
100 \\
100 \\
100 \\
91 \\
99 \\
\end{array}$ & \begin{tabular}{|c|}
.0012 \\
.0063 \\
.0084 \\
.0020 \\
$8.7 \times 10^{-7}$ \\
$1.57 \times 10^{-6}$ \\
1.57
\end{tabular} & $\begin{array}{l}13.8 \\
17.6 \\
13.2 \\
15.3 \\
15.3 \\
15.1 \\
115 .\end{array}$ & \begin{tabular}{|l|}
63.92 \\
31.2 \\
29.0 \\
43.3 \\
26.2 \\
27.2 \\
\end{tabular} & $\begin{array}{l}230.1 \\
34.9 \\
31.4 \\
72.7 \\
33.1 \\
24.3 \\
\end{array}$ & \begin{tabular}{r|}
11.5 \\
3.1 \\
3.8 \\
2.5 \\
1.6 \\
4.2 \\
\end{tabular} & $\begin{array}{r}24.0 \\
3.3 \\
4.3 \\
3.7 \\
2.8 \\
4.5 \\
\end{array}$ & $\begin{array}{l}1.28 \\
1.06 \\
1.03 \\
1.48 \\
1.75 \\
1.07 \\
\end{array}$ & \begin{tabular}{|l}
$\mathrm{VH}$ \\
$\mathrm{M}$ \\
$\mathrm{M}-\mathrm{H}$ \\
$\mathrm{M}$ \\
$\mathrm{L}-\mathrm{BH}$ \\
$\mathrm{H}$ \\
\end{tabular} & $\begin{array}{l}\mathrm{SC} \\
\mathrm{T} \\
\mathrm{T} \\
\mathrm{T} \\
\mathrm{SL} \\
\mathrm{SL} \\
\end{array}$ \\
\hline
\end{tabular}




\section{Notes for Tables AI, AII and AIII.}

1. The data reported in this table pertain to specimens frozen in the laboratory under conditions which include the following:

a. Degree of saturation before freezing equal to or greater than $85 \%$.

b. Molded dry unit weight equal to or greater than $95 \%$ of the applicable maximum standard.

c. Rate of penetration of the $32^{\circ} \mathrm{F}$ isotherm approximately $1 / 4$ to $1 / 2$ in./day.

d. Load pressure:

Table AI $-0.5 \mathrm{psi}$

Table AII $-0.5 \mathrm{psi}$

Table AIII - 0.073 psi (1/4-in. steel plate only)

e. Height of molded specimen approximately 6 in.

f. Free water supply at base of specimen (water maintained at approximately $38^{\circ} \mathrm{F}$ ).

The specimens are listed in order of increasing percentage finer than 0.02-mm grain size within each soil classification group.

2. U.S. Army Engineer Waterways Experiment Station, The Unified Soil Classification System. Technical Memorandum No. 3-357, vol. 1, Vicksburg, Mississippi, revised 1960.

3. Gradation coefficients (for reference - see note 2):

$$
\begin{aligned}
& C_{u}=\text { coefficient of uniformity }=\frac{D_{60}}{D_{10}} \\
& C_{c}=\text { coefficient of curvature }=\frac{\left(D_{30}\right)^{2}}{\left(D_{60}\right)\left(D_{10}\right)}
\end{aligned}
$$

4. Atterberg limits tests performed on material passing the U.S. Standard no. 40 sieve. If no limits are shown, material is nonplastic. LL = Liquid limit; PI = Plasticity index:

5. The maximum dry unit weight and the optimum moisture content are shown for the natural soil of each specimen. The type of compaction test used in each case is indicated by the letter in parentheses listed alongside the maximum dry unit weight:

a. AASHO T99-57' Method A.

b. Providence Vibrated Density Test.

c. AASHO T180-57 Method D.

d. AASHO T180-57 Method A.

e. Harvard Miniature Compaction Test.

6. Degree of saturation in percent at start of freezing test. Remolded specimens allowed to drain for 24 hours just prior to freezing.

7. Permeability tested with de-aired water under falling head and corrected to $10^{\circ} \mathrm{C}$. Values reported are for corresponding specimen void ratios.

8. Based on the original height of the frozen portion.

9. Rate of heave - the average rate of heave in millimeters per day, determined from a representative portion of the plot of heave versus time, in which the slope is relatively constant and during which the penetration of the $32^{\circ} \mathrm{F}$ isotherm is relatively linear and between $1 / 4$-in. and $1 / 2-$ in./day. Rate of heave is averaged over as much of the heave versus the time plot as practicable, but the minimum number of consecutive days used for a determination is five. Maximum rate - the average of the three highest, not necessarily consecutive, daily heave rates. 


\section{Notes for Tables AI, AII and AII (Cont'd).}

10. Heave rate variability index $=$ Maximum heave rate/Average heave rate.

11. The following tentative scales of average and maximum rates of heave have been adopted for rates of freezing between $1 / 4$-in. and $1 / 2$-in./day:

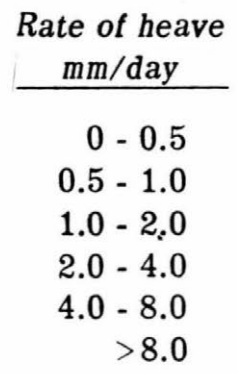

\begin{tabular}{ll}
\multicolumn{3}{c}{ Relative frost } \\
susceptibility classi \\
\hline Negligible & N \\
Very low & VL \\
Low & L \\
Medium & M \\
High & H \\
Very high & VH
\end{tabular}

12. Symbols indicate different types of specimen containers used during the studies:

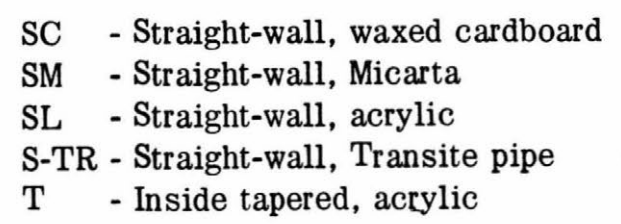

13. The specimens listed in supplementary Table AII do not fulfill requirements given under Note $1 \mathrm{a}$ and $1 \mathrm{~b}$ above; otherwise all other notes apply.

14. The specimens listed in Table AII have been tested under a load pressure of $0.073 \mathrm{psi}$, and may or may not fulfill $1 \mathrm{a}$ and $1 \mathrm{~b}$; otherwise all other notes apply. 
DOCUMENT CONTROL DATA - R \& D

(Security classification of title, body of abstract and indexing annotation must be entered when the overall report is classified) 1. ORIGINA TING ACTIVITY (Corporate author)

U.S. Army Cold Regions Research and Engineering Laboratory

Hanover, New Hampshire 03755

2a. REPORT SECURITY CLASSIFICATION

REPORT TITLE

FREEZING TEST FOR EVALUATING RELATIVE FROST SUSCEPTIBILITY OF VARIOUS SOILS

4. DESCRIPTIVE NOTES (Type of report and inclusive dates)

5. AUTHOR(S) (First name, middle initial, last name)

Chester W. Kaplar

\begin{tabular}{|c|c|c|}
\hline $\begin{array}{l}\text { 6. REPORT DATE } \\
\text { June } 1974\end{array}$ & $\begin{array}{c}\text { 7a. TOTAL NO. OF PAGES } \\
40\end{array}$ & $\begin{array}{c}\text { 7b. NO. OF REFS } \\
14 \\
\end{array}$ \\
\hline $\begin{array}{l}\text { 8a. CONTRACT OR GRANT NO. } \\
\text { b. PROJECT NO. }\end{array}$ & $\begin{array}{r}\text { 9a. ORIGINATOR'S REPOR } \\
\text { Technic }\end{array}$ & eport 250 \\
\hline $\begin{array}{l}\text { c. } \\
\text { d. }\end{array}$ & $\begin{array}{l}\text { 9b. OTHER REPORT NO(S) } \\
\text { this roport) }\end{array}$ & her numbers that may bo as siened \\
\hline
\end{tabular}

10. DISTRIBUTION STATEMENT

Approved for public release; distribution unlimited.

11. SUPPLEMENTARY NOTES

12. SPONSORING MILITARY ACTIVITY

Directorate of Military Construction

Office of the Chief of Engineers

13. ABSTRACT

This report presents a description of the equipment and procedures used in the laboratory test of the relative frost susceptibility of different soils on Corps of Engineers construction projects and includes typical results of freezing tests of natural soils. The test utilizes a slow unidirectional freezing of a 6-in. high, remolded or undisturbed soil specimen with water available at the base (open system). The heaving rate measured during the test is used as the basis for classification of the frost susceptibility potential of the soil. This type of test, which measures heaving rate, is considered most versatile and adaptable for evaluating the effects of numerous soil parameters on frost heave.

14. Key Words

Frost heave

Frost susceptibility tests

Frozen soil tests

Soil freezing tests 